Supporting Information for

\title{
Deep Learning Enables Discovery of a Short Nuclear Targeting Peptide for Efficient Delivery of Antisense Oligomers
}

Authors: Eva M. López-Vidal ${ }^{1 \dagger}$, Carly K. Schissel ${ }^{1 \dagger}$, Somesh Mohapatra ${ }^{2}$, Kamela Bellovoda ${ }^{3}$, Chia-Ling Wu ${ }^{3}$, Jenna A. Wood $^{3}$, Annika B. Malmberg ${ }^{3}$, Andrei Loas ${ }^{1}$, Rafael Gómez-Bombarelli2 ${ }^{*}$, Bradley L. Pentelute ${ }^{1,4,5,6^{*}}$

\section{Affiliations:}

${ }^{1}$ Massachusetts Institute of Technology, Department of Chemistry, 77 Massachusetts Avenue, Cambridge, MA 02139, USA

${ }^{2}$ Massachusetts Institute of Technology, Department of Materials Science and Engineering, 77

Massachusetts Avenue, Cambridge, MA 02139, USA

${ }^{3}$ Sarepta Therapeutics, 215 First Street, Cambridge, MA 02142, USA

${ }^{4}$ The Koch Institute for Integrative Cancer Research, Massachusetts Institute of Technology, 500 Main Street, Cambridge, MA 02142, USA

${ }^{5}$ Center for Environmental Health Sciences, Massachusetts Institute of Technology, 77 Massachusetts Avenue, Cambridge, MA 02139, USA

${ }^{6}$ Broad Institute of MIT and Harvard, 415 Main Street, Cambridge, MA 02142, USA

$†$ These authors contributed equally to this work.

*Correspondence to: blp@mit.edu, rafagb@mit.edu

\section{This PDF file includes:}

Supplementary materials and methods

Figures S1 to S14

Tables S1 to S3 
Table of Contents

1. Materials. 3

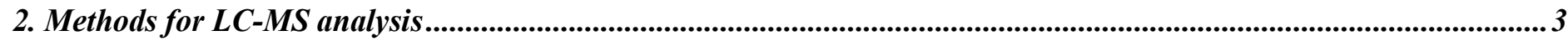

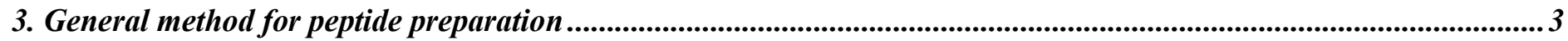

Semi-automated fast-flow peptide synthesis ..................................................................................................................................

Functionalization of the peptides with azide at the N-terminus ................................................................................................ 4

Peptides cleavage and deprotection ........................................................................................................................................... 4

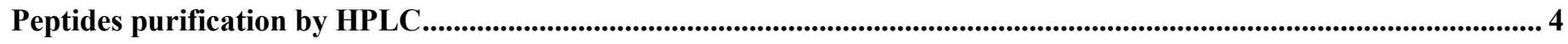

Peptides purification using reversed-phase flash chromatography (Biotage ${ }^{\square}$ ).................................................................... 4

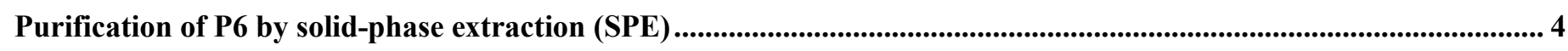

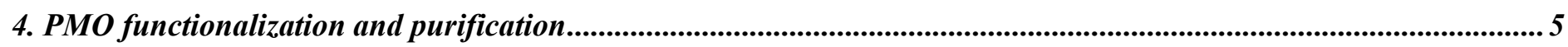

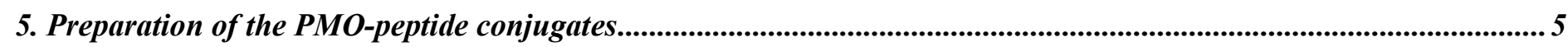

PMO-peptide conjugation using copper-free azide-alkyne click chemistry ..........................................................................5

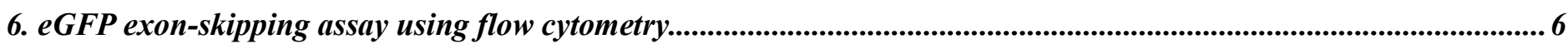

Exon skipping activity of the conjugates with the peptides predicted by machine learning and analogs of P6............... 6

Exon skipping activity of PMO-P6 and PMO-P12 using endocytosis inhibitors..................................................................... 9

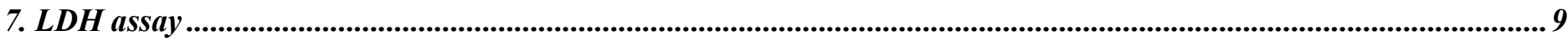

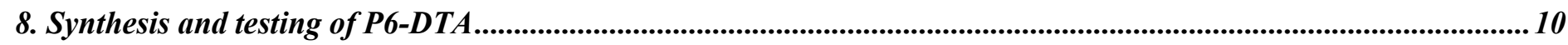

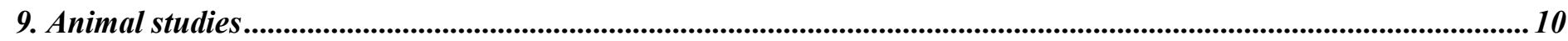

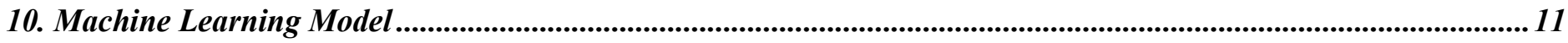

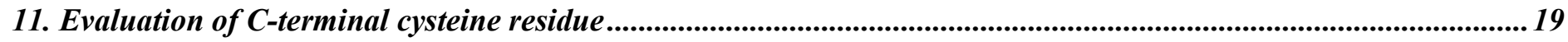

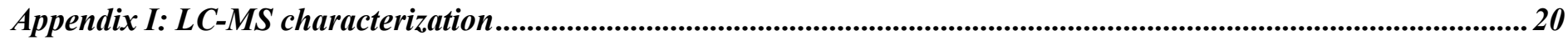




\section{Materials}

H-Rink Amide-ChemMatrix resin was obtained from PCAS BioMatrix Inc. (St-Jean-sur-Richelieu, Quebec, Canada). 1[Bis(dimethylamino)methylene]-1 $H$-1,2,3-triazolo[4,5-b]pyridinium-3-oxid-hexafluorophosphate (HATU) was purchased from P3 BioSystems. 5-azidopentanoic acid was obtained from Bachem. Fmoc- $\beta$-Ala-OH, Fmoc-6-amino hexanoic acid and 2-(1Hbenzotriazol-1-yl)-1,1,3,3-tetramethyluronium hexafluorophosphate (HBTU) were purchased from Chem-Impex International. Fmoc-Ala-OH, Fmoc-Cys(Trt)-OH, Fmoc-Gly-OH, Fmoc-His(Trt)-OH, Fmoc-Lys(Boc)-OH, Fmoc-Leu-OH, Fmoc-Met-OH, Fmoc-Asn(Trt)-OH, Fmoc-Pro-OH, Fmoc-Gln(Trt)-OH, Fmoc-Arg(Pbf)-OH, Fmoc-Thr(tBu)-OH, Fmoc-Trp(Boc)-OH and Fmoc-Ser(Trt)-OH were purchased from Novabiochem. Peptide synthesis-grade $N, N$-dimethylformamide (DMF), dichloromethane (DCM), diethyl ether and HPLC-grade acetonitrile were obtained from VWR International. All other reagents, such as dibenzocyclooctyne-acid (DBCO acid), ammonium hydroxide $\left(\mathrm{NH}_{4} \mathrm{OH}\right)$, piperidine, dimethyl sulfoxide (DMSO), $N, N$ diisopropylethylamine (DIEA), trifluoroacetic acid (TFA), thioanisole or triisopropylsilane (TIPS), were purchased from SigmaAldrich. Milli-Q water was used exclusively. Minimum essential medium (MEM) and tripsin-EDTA (0.25\%) were obtained from Gibco. Penicillin Streptomycin was bought from ThermoFisher Scientific. Fetal bovine serum (FBS) was purchased from MilliporeSigma. Phosphate buffered saline (PBS) 1X was obtained from Corning. Propidium iodide was purchased from Invitrogen. DMEM-high glucose was obtained from HyClone. HeLa-654 cells were obtained from the University of North Carolina Tissue Culture Core facility. Human renal proximal tubule epithelial (TH1 RPTEC) cells were bought from Kerafast. PMO IVS2-654 was provided by Sarepta Therapeutics. For the LDH assay, the CytoTox 96® non-Radioactive cytotoxicity assay kit was purchased from Promega.

\section{Methods for LC-MS analysis}

LC-MS analyses were performed on different spectrometers and using different methods.

Agilent 6520 ESI-Q-TOF mass spectrometer equipped with a C18 Zorbax column (300SB C18, $150 \mathrm{~mm} \times 2.1 \mathrm{~mm}$ ID, $5 \mu \mathrm{m} 300$ A silica). Mobile phases were: $0.1 \%$ formic acid in water (solvent A) and $0.1 \%$ formic acid in acetonitrile (solvent B). The following LC-MS method was used for characterization: 1\% B from 0 to 2 min, linear ramp from 1\% B to $61 \% \mathrm{~B}$ from 2 to 11 min, $61 \% \mathrm{~B}$ to $95 \% \mathrm{~B}$ from 11 to $12 \mathrm{~min}$ and finally $3 \mathrm{~min}$ of post-time at $1 \% \mathrm{~B}$ for equilibration, flow rate: $0.6 \mathrm{~mL} / \mathrm{min}$.

Agilent 6550 ESI-iFunnelQ-TOF mass spectrometer equipped with a C18 column (Phenomenex Luna C18(2), $150 \mathrm{~mm}$ x $0.5 \mathrm{~mm}$ ID (capillary), $3 \mu \mathrm{m} 100 \AA$ silica). Mobile phases were: $0.1 \%$ formic acid in water (solvent A) and $0.1 \%$ formic acid in acetonitrile (solvent B). The following LC-MS method was used for characterization: $1 \% \mathrm{~B}$ and ramping to $61 \% \mathrm{~B}$ over 12 minutes. MS was on from 4 to 14 minutes. Flow rate was $50 \mu \mathrm{L} / \mathrm{min}$.

Agilent 6550 ESI-iFunnelQ-TOF mass spectrometer equipped with a C4 column (Phenomenex Jupiter C4, $150 \mathrm{~mm} \times 1.0 \mathrm{~mm}$ ID, $5 \mu \mathrm{m} 300 \AA$ silica). Mobile phases were: $0.1 \%$ formic acid in water (solvent A) and $0.1 \%$ formic acid in acetonitrile (solvent B). The following LC-MS method was used for characterization: 1\% B and ramping to $61 \%$ B over 10 minutes. MS was on from 4 to 12 minutes. Flow rate was $100 \mu \mathrm{L} / \mathrm{min}$.

Chromatograms were obtained using one of the previous methods unless otherwise noted. All data were processed using Agilent MassHunter software package. Y-axis in all chromatograms shown represents total ion current (TIC) unless noted.

\section{General method for peptide preparation}

\section{Semi-automated fast-flow peptide synthesis}

Peptides P1- P19 were synthesized on a $0.1 \mathrm{mmol}$ scale using semi-automated fast-flow peptide synthesis. A $140 \mathrm{mg}$ portion of H-Rink Amid-ChemMatrix was loaded into the reactor and maintained at $70{ }^{\circ} \mathrm{C}$. All reagents and solvents were flowed through a stainless-steel loop maintained at $70{ }^{\circ} \mathrm{C}$ before introduction into the reactor. For each coupling, $2.5 \mathrm{~mL}$ of HATU $(0.38 \mathrm{M}$ in DMF) and amino acid $(0.1 \mathrm{mmol})$ were mixed with $0.5 \mathrm{~mL}$ of DIEA and delivered to the reactor at $6 \mathrm{~mL} / \mathrm{min}$ followed by washing with DMF for 1 minute at $20 \mathrm{~mL} / \mathrm{min}$. Fmoc removal was accomplished washing with $20 \%$ (v/v) piperidine in DMF for 20 seconds at $20 \mathrm{~mL} / \mathrm{min}$. Between each step and at the end of the peptide synthesis, DMF (1 minute at $20 \mathrm{~mL} / \mathrm{min}$ ) was used to wash out the reactor. 
Functionalization of the peptides with azide at the N-terminus

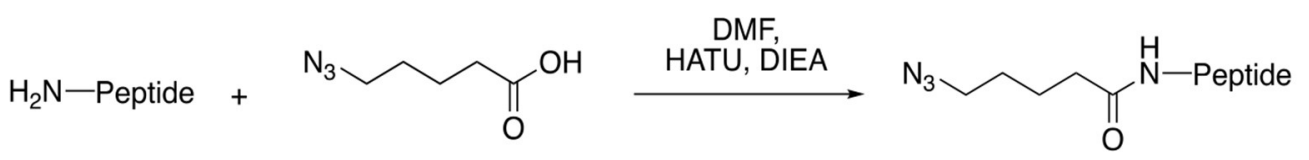

Fig. S1: Reaction scheme for the functionalization of the peptides as azide at the N-terminus.

To cap the peptides with 5-azidopentanoic acid, $50 \mathrm{mg}$ of each peptide attached to the resin (P1- P7) were incubated for 1 hour at room temperature with 5-azidopentanoic acid $(0.25 \mathrm{mmol})$ dissolved in $0.625 \mathrm{~mL}$ of HATU $(0.38 \mathrm{M}$ in DMF) and $0.125 \mathrm{~mL}$ of DIEA. For peptides P8- P19, $150 \mathrm{mg}$ of each peptide attached to the resin were incubated for 1 hour at room temperature with 5azidopentanoic acid $(0.75 \mathrm{mmol})$ dissolved in $1.875 \mathrm{~mL}$ of HATU $(0.38 \mathrm{M}$ in DMF) and $0.375 \mathrm{~mL}$ of DIEA. After completion of the synthesis, the resins were washed 3 times with DCM and dried under vacuum.

\section{Peptides cleavage and deprotection}

After functionalization of $50 \mathrm{mg}$ of the peptide-resin, each peptide was subjected to simultaneous global side-chain deprotection and cleavage from resin by treatment with $1.25 \mathrm{~mL}$ of a a mixture of $94 \%$ trifluoroacetic acid (TFA), $2.5 \%$ thioanisole, $2.5 \%$ water and $1 \%$ triisopropylsilane (TIPS) (v/v) and the mixture was kept for 2 hours at room temperature.

Afterwards, $10 \mathrm{~mL}$ of cold diethyl ether (chilled at $-80{ }^{\circ} \mathrm{C}$ ) were added to precipitate and wash each of the peptides. The crude product was pelleted through centrifugation for 3 minutes at 4,000 rpm and the diethyl ether was decanted. Precipitation with cold diethyl ether $(4 \mathrm{~mL})$ and centrifugation was repeated two more times. After the third wash, the pellet was dissolved in a mixture $(4 \mathrm{~mL}$ ) of $50 \%$ water, $50 \%$ acetonitrile and $0.1 \%$ TFA, vortexed and filtered through a fritted syringe to remove the resin and lyophilized.

In the case of the cleavage of $150 \mathrm{mg}$ of functionalized peptide-resin, $4 \mathrm{~mL}$ of the same cleavage cocktail were used and after cleavage, the triple quantities of cold diethyl ether were used to precipitate and wash the peptides.

\section{Peptides purification by HPLC}

The peptides were dissolved in water and acetonitrile containing $0.1 \%$ TFA, filtered through a $0.22 \mu \mathrm{m}$ nylon filter and purified by mass-directed semi-preparative reversed-phase HPLC. Solvent A was water with $0.1 \%$ of TFA and solvent B was acetonitrile with $0.1 \%$ of TFA. The peptides were purified on an Agilent Zorbax 300SB-C18 column: 9.4 x $250 \mathrm{~mm}, 5 \mu \mathrm{m}$. Using mass data about each fraction from the instrument, only pure fractions were pooled and lyophilized. The purity of the fraction pool was confirmed by LC-MS. For peptides P1- P7, a linear gradient that changed at a rate of $1 \% /$ min was used starting with $1 \%$ B and finishing with $61 \%$ B over a period of 60 minutes. For peptides P8- P19 the purification started with 1\% B and finished with $40 \%$ B over a period of 60 minutes.

\section{Peptides purification using reversed-phase flash chromatography $\left(\right.$ Biotage $\left.^{\circledR}\right)$}

Peptides P6, P8- P18 were purified using reversed-phase flash chromatography (Biotage $\left.{ }^{\circledR}\right)$. The peptides were dissolved in water and acetonitrile containing $0.1 \%$ TFA and purified using reversed-phase flash chromatography. Solvent A was water with $0.1 \%$ of TFA and solvent B was acetonitrile with $0.1 \%$ of TFA. The peptides were purified using a SNAP Bio C18 $300 \AA$, $10 \mathrm{~g}$ column. A linear gradient from $1 \% \mathrm{~B}$ to $10 \% \mathrm{~B}$ was used during 4 column volumes, $10 \% \mathrm{~B}$ to $50 \% \mathrm{~B}$ during 15 column volumes followed by an equilibration step during 2 column volumes at $50 \% \mathrm{~B}$. Using UV data of each fraction from the instrument, only pure fractions were pooled and lyophilized. The purity of the fraction pool was confirmed by LC-MS.

\section{Purification of P6 by solid-phase extraction (SPE)}

Peptide P6 could also be purified using SPE. The column used was SUPELCO LC-18 SPE, 1g, $6 \mathrm{~mL}, \mathrm{C} 18$. The column was conditioned with $12 \mathrm{~mL}$ of $\mathrm{MeOH}$ and then with $12 \mathrm{~mL}$ of $99 \%$ water and $1 \%$ acetonitrile containing $0.1 \%$ TFA. Peptide P6 (52 $\mathrm{mg}$ ) was dissolved in $6 \mathrm{~mL}$ of $99 \%$ water and $1 \%$ acetonitrile containing $0.1 \%$ of TFA. The column was washed with $12 \mathrm{~mL}$ of $99 \%$ water and $1 \%$ acetonitrile containing $0.1 \%$ of TFA and then the compound was eluted with $12 \mathrm{~mL}$ of $30 \%$ water and $70 \%$ acetonitrile containing $0.1 \%$ of TFA. The eluted fraction was collected and the purity of the fraction pool was confirmed by LCMS. After lyophilization $41 \mathrm{mg}$ of P6 were obtained. 


\section{PMO functionalization and purification}

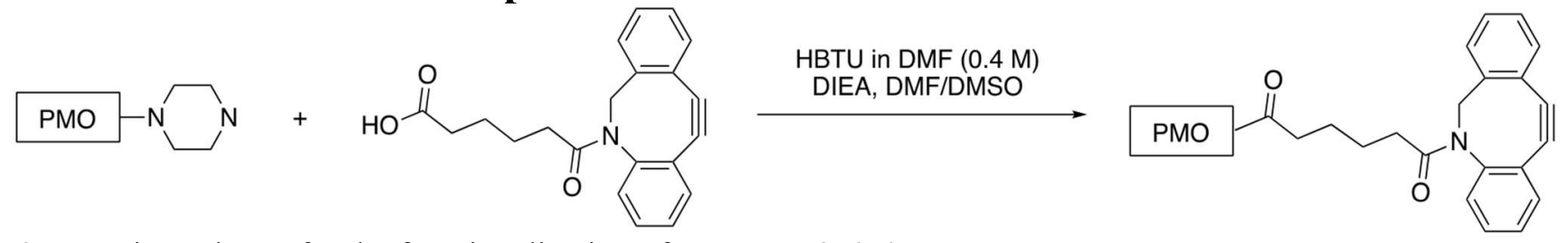

Fig. S2: Reaction scheme for the functionalization of PMO IVS2-654 as DBCO.

PMO IVS2-654 was functionalized with DBCO acid. The DBCO acid $(10 \mathrm{mg})$ was dissolved in $80 \mu \mathrm{L}$ of amine-free DMF. Then, $74 \mu \mathrm{L}$ of HBTU ( $0.4 \mathrm{M}$ in amine-free DMF) and $5.6 \mu \mathrm{L}$ of DIEA were added to the DBCO acid solution. Separately, $100 \mathrm{mg}$ of PMO IVS2-654 were dissolved in $300 \mu \mathrm{L}$ of DMSO. The DBCO solution was added to the PMO solution and mixed gently. The reaction was left to react for 30 minutes. Afterwards, the reaction was quenched with $2 \mathrm{~mL}$ of water and $4 \mathrm{~mL}$ of $\mathrm{NH}_{4} \mathrm{OH}$ and left for 20 minutes. The reaction mixture was diluted with $40 \mathrm{~mL}$ of water/acetonitrile (95:5) and purified by mass-directed preparative reversed-phase HPLC. Solvent A was water and solvent B was acetonitrile. The PMO functionalized with DBCO was purified on an Agilent Zorbax 300SB-C3 column: $21.2 \times 100 \mathrm{~mm}, 5 \mu \mathrm{m}$. A linear gradient was used starting with 2\% B and finishing with $60 \% \mathrm{~B}$ over a period of 70 minutes. Using mass data about each fraction from the instrument, only pure fractions were pooled and lyophilized. The purity of the fraction pool was confirmed by LC-MS.

\section{Preparation of the PMO-peptide conjugates}

\section{PMO-peptide conjugation using copper-free azide-alkyne click chemistry}

Peptide conjugates were synthesized using copper-free azide-alkyne 1,3-dipolar cycloaddition in water. PMO-DBCO was dissolved in water at $5 \mathrm{mM}$ concentration (determined gravimetrically). The peptides were dissolved in water at $5 \mathrm{mM}$ concentration (determined gravimetrically; the molecular weight was calculated to include 1 trifluoroacetate counter ion per lysine and arginine residue). In a microcentrifuge tube, equal volumes of PMO-DBCO solution and peptide solution were mixed (volumes between $12 \mu \mathrm{L}$ and $100 \mu \mathrm{L}$ depending on the reaction) and the resulting mixtures were vortexed. The reactions took place within a period of 1 day and they were followed by LC-MS until $\sim 95 \%$ consumption of peptide was observed. The PMOpeptide conjugates were lyophilized.
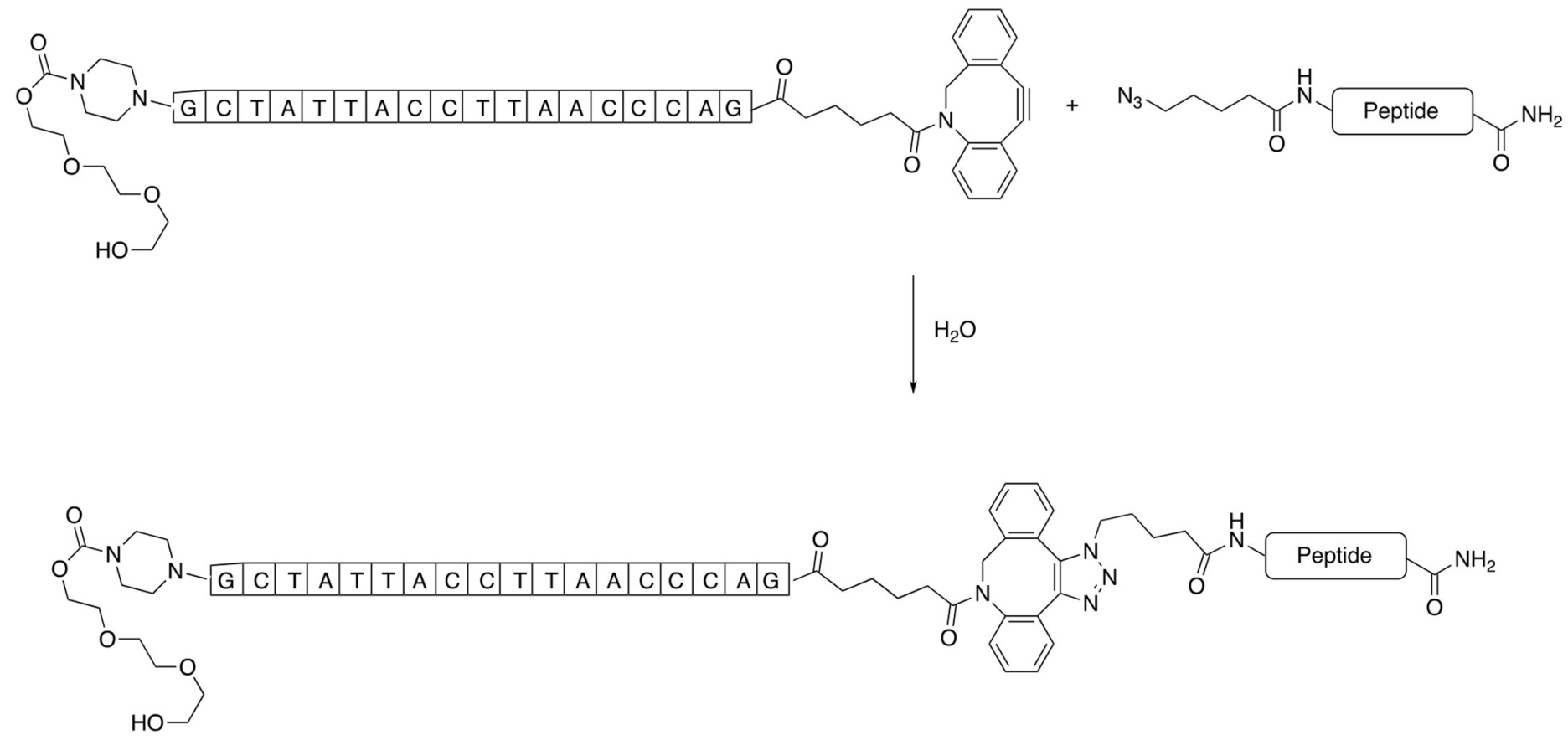

Fig. S3: Reaction scheme for the conjugation of PMO to peptide through copper-free click chemistry. 
PMO-peptide conjugates purification by HPLC

Each PMO-peptide conjugate was dissolved in water. The solution was filtered through a $0.22 \mu \mathrm{m}$ nylon filter and purified by mass-directed semi-preparative reversed-phase HPLC. Solvent A was water with $100 \mathrm{mM}$ ammonium acetate (pH 7.2) and solvent B was acetonitrile.

Conjugates PMO-P1 to PMO-P7 were dissolved in water and purified on an Agilent Zorbax 300SB-C3 column: 9.4 x $250 \mathrm{~mm}$, $5 \mu \mathrm{m}$. A linear gradient that changed at a rate of $1 \%$ per min was used starting with $1 \% \mathrm{~B}$ and finishing with $61 \% \mathrm{~B}$ over a period of 60 minutes. Using mass data about each fraction from the instrument, only pure fractions were pooled and lyophilized. The purity of the fraction pool was confirmed by LC-MS. PMO-peptide conjugates were obtained as their acetate salts.

Conjugates PMO-P8 to PMO-P19 were dissolved in water and purified on an Agilent Zorbax 300SB-C3 column: $2.1 \mathrm{x} 150 \mathrm{~mm}$, $5 \mu \mathrm{m}$. A linear gradient that changed at a rate of $2 \%$ per min was used starting with $1 \%$ B and finishing with $61 \%$ B over a period of 30 minutes. Using mass data about each fraction from the instrument, only pure fractions were pooled and lyophilized. The purity of the fraction pool was confirmed by LC-MS. PMO-peptide conjugates were obtained as their acetate salts.

\section{6. eGFP exon-skipping assay using flow cytometry}

Exon skipping activity of the conjugates with the peptides predicted by machine learning and analogs of P6 HeLa654 cells were maintained in MEM (Minimum Essential Medium) supplemented with $10 \%$ (v/v) of fetal bovine serum (FBS) and $1 \%(\mathrm{v} / \mathrm{v})$ of penicillin-streptomycin (Pen Strep) at $37{ }^{\circ} \mathrm{C}$ and $5 \% \mathrm{CO}_{2} .24$ hours before treatment, HeLa654 cells were plated at a density of 8,000 cells per well in a 96-well plate. The next day, fresh $1 \mathrm{mM}$ stocks of each of the PMO-peptide conjugates in PBS (1X) were prepared. The exact concentration of the stock solutions was determined by measuring the absorbance at $260 \mathrm{~nm}$ and a PMO extinction coefficient of $168,700 \mathrm{~L} \mathrm{~mol}^{-1} \mathrm{~cm}^{-1}$. The growth media was aspirated from the cells and treatment media consisting of each respective conjugate (5 $\mu \mathrm{M}$ concentration) in MEM supplemented with $10 \% \mathrm{FBS}$ and $1 \%$ Pen Strep was added. The cells were incubated with treatment-containing media for 22 hours at $37{ }^{\circ} \mathrm{C}$ and $5 \% \mathrm{CO}_{2}$. Next, the treatment media was aspirated. Trypsin-EDTA $(0.25 \%)(20 \mu \mathrm{L})$ was added to the cells and incubated for $10 \mathrm{~min}$ at $37{ }^{\circ} \mathrm{C}$ and $5 \% \mathrm{CO}_{2}$. To quench the trypsin, $80 \mu \mathrm{L}$ of MEM supplemented with 10\% (v/v) FBS and 1\% (v/v) Pen Strep was added to each well. The dissociated cells in media were transferred with a multichannel pipette to a polypropylene v-bottom 96 -well plate (Falcon) and centrifuged at 500 rcf (relative centrifugal force) for $3 \mathrm{~min}$. The supernatant was removed, the cell pellets were resuspended with $200 \mu \mathrm{L}$ of PBS $(1 \mathrm{X})$, and the plate was centrifuged again at $500 \mathrm{rcf}$ for $3 \mathrm{~min}$. The supernatant was again removed and the pellets were resuspended in $300 \mu \mathrm{L}$ of PBS (1X) with $2 \% \mathrm{FBS}(\mathrm{v} / \mathrm{v})$ and $0.2 \%$ of a solution of propidium iodide in water. Fluorescence intensity was analyzed by flow cytometry on a BD LSRII flow cytometer. Gates were applied to the data to ensure that cells that were highly positive for propidium iodide or exhibited forward/side scatter readings that were sufficiently different from the main cell population were excluded. The mean fluorescence intensity was calculated for each sample and normalized with respect to the fluorescence of the cells treated with unconjugated PMO. For each PMO-peptide conjugate, the mean fluorescence intensity was averaged across biological replicates. 


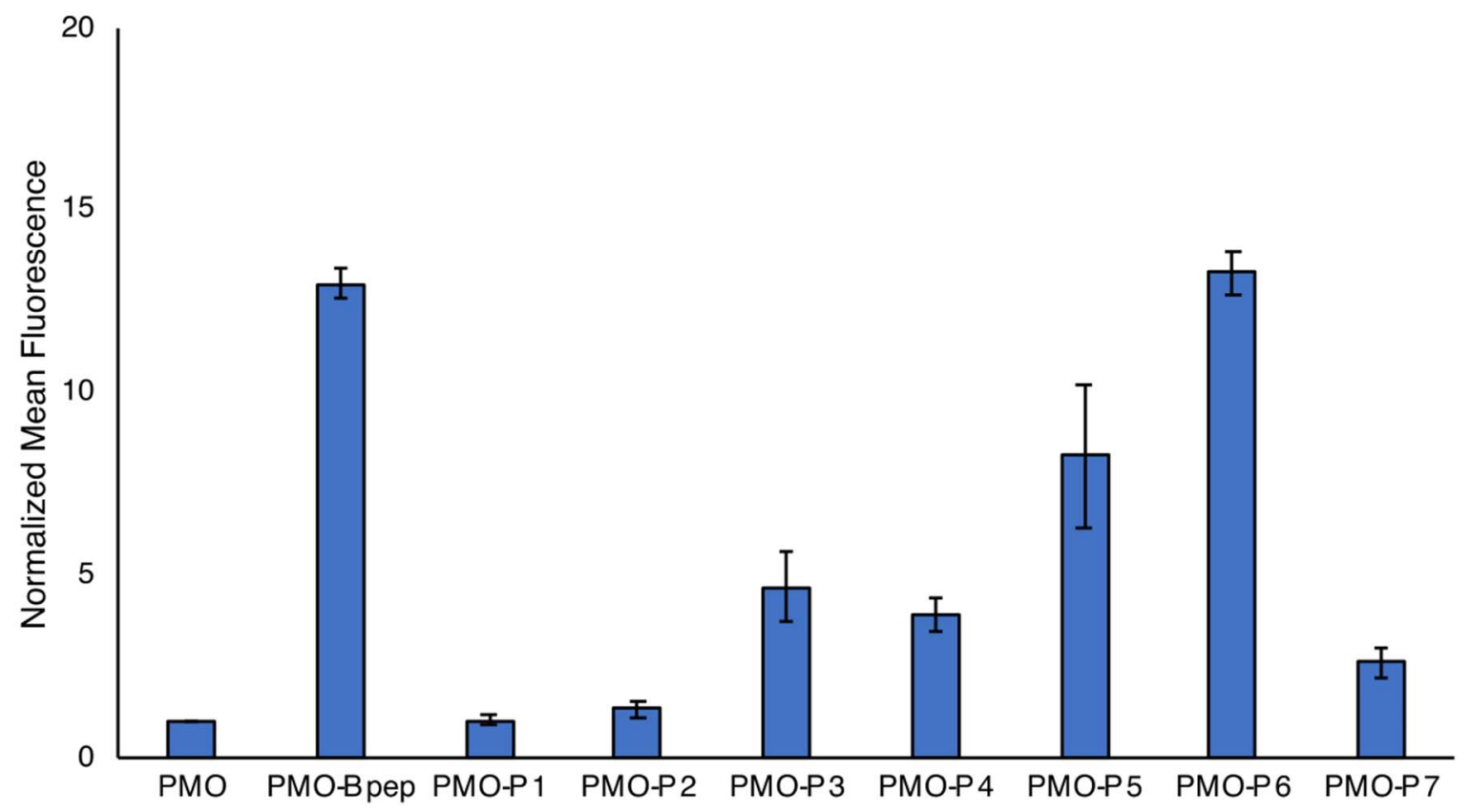

Fig. S4. Activity (eGFP assay) of the PMO-peptide conjugates with peptides P1- P7 measured in one biological replicate at a concentration of $5 \mu \mathrm{M}$ for each PMO-peptide conjugate. The eGFP fluorescence was normalized with respect to the PMO alone condition.

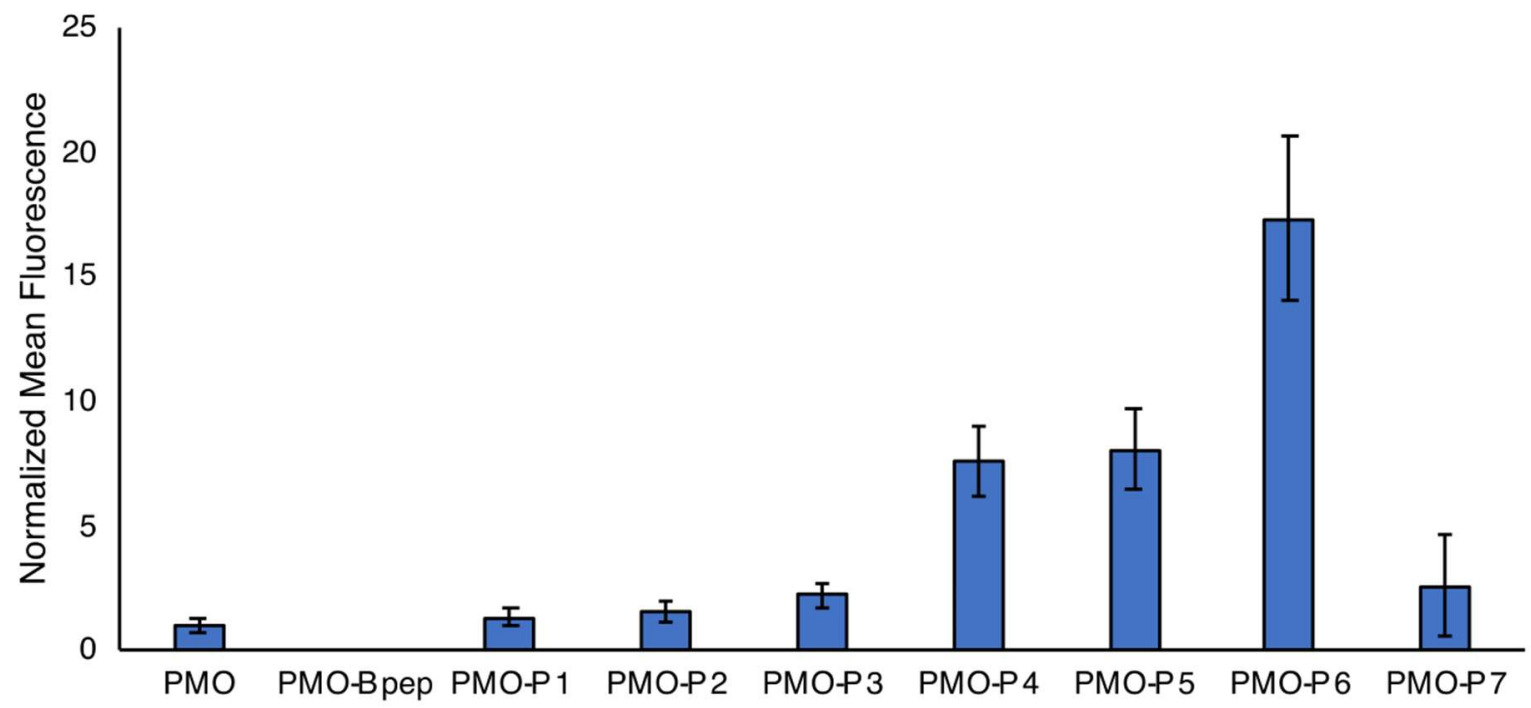

Fig. S5. Activity (eGFP assay) of the PMO-peptide conjugates with peptides P1- P7 measured in one biological replicate at a concentration of $5 \mu \mathrm{M}$ for each PMO-peptide conjugate. The eGFP fluorescence was normalized with respect to the PMO alone condition. 


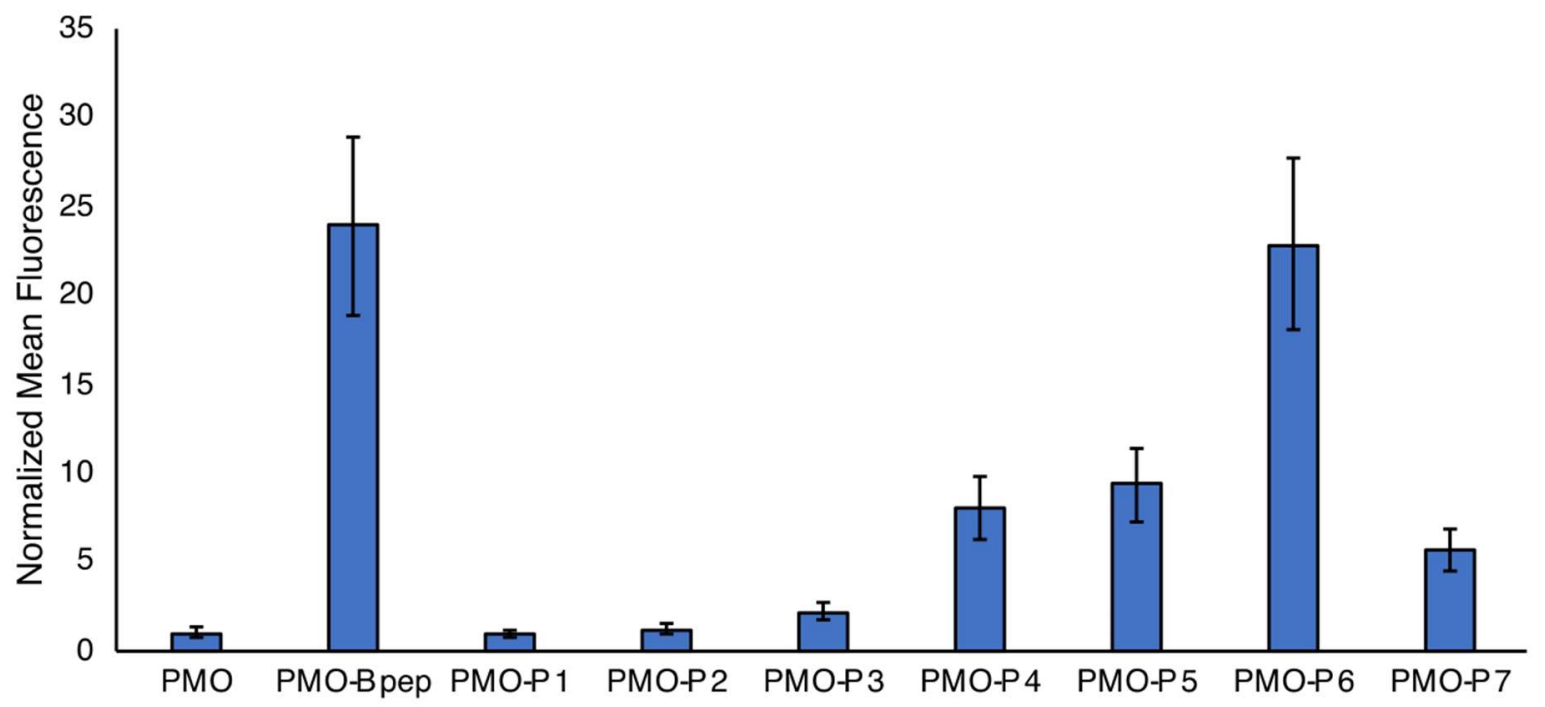

Fig. S6. Activity (eGFP assay) of the PMO-peptide conjugates with peptides P1- P7 measured in one biological replicate at a concentration of $5 \mu \mathrm{M}$ for each PMO-peptide conjugate. The eGFP fluorescence was normalized with respect to the PMO alone condition.

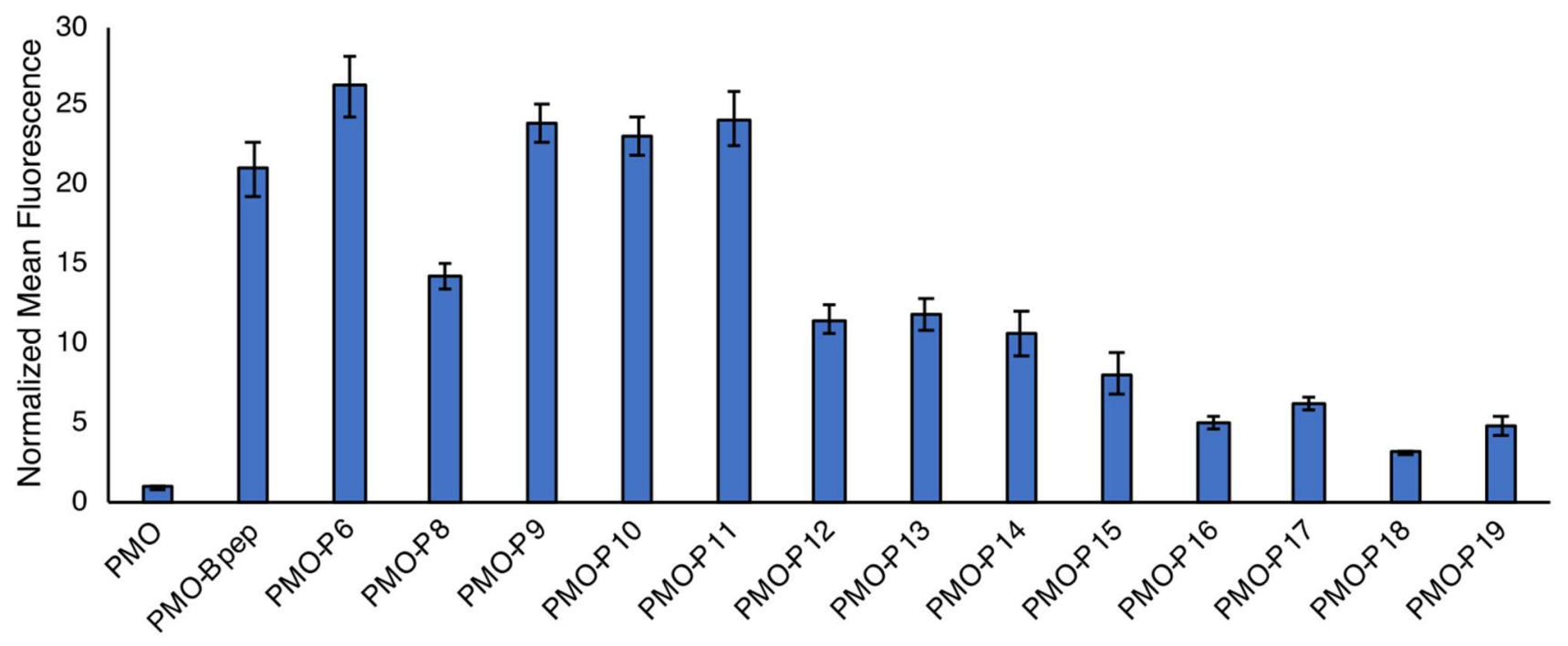

Fig. S7. Activity (eGFP assay) of the PMO-peptide conjugates with peptides P6- P19 measured in one biological replicate at a concentration of $5 \mu \mathrm{M}$ for each PMO-peptide conjugate. The eGFP fluorescence was normalized with respect to the PMO alone condition. 


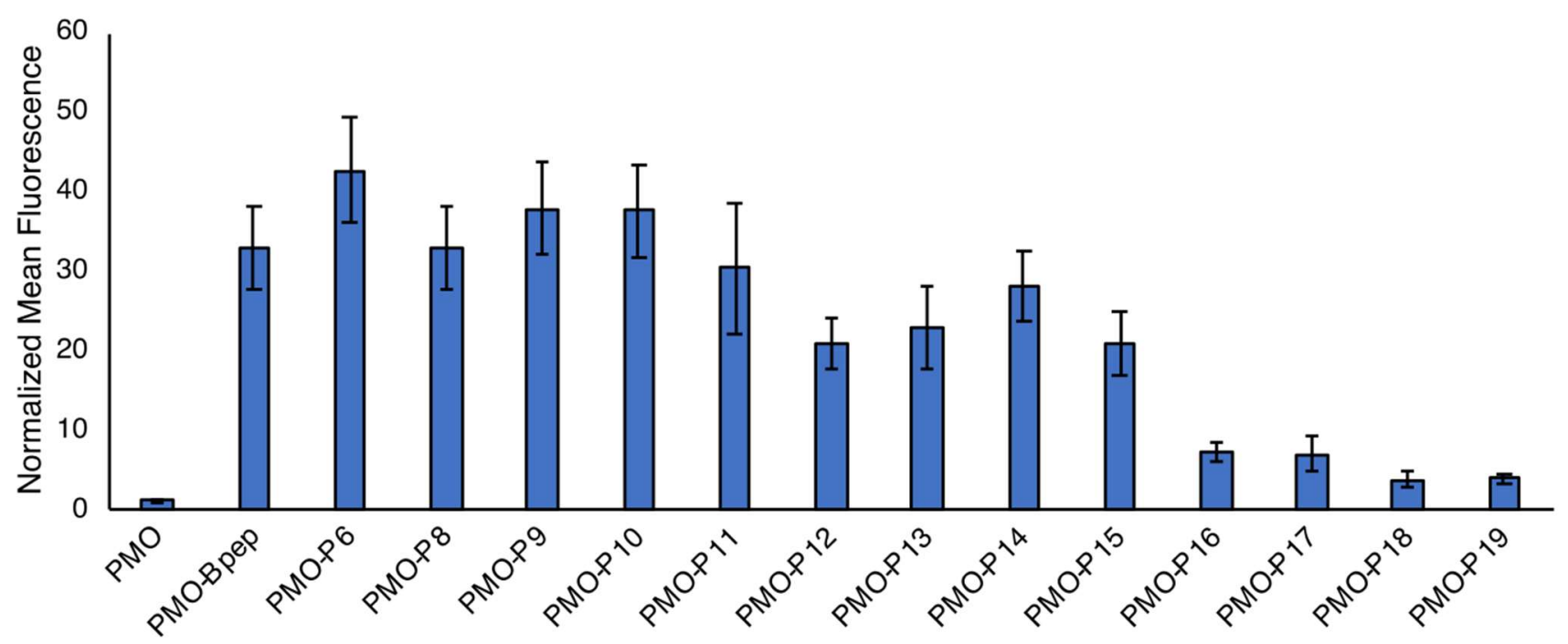

Fig. S8. Activity (eGFP assay) of the PMO-peptide conjugates with peptides P6- P19 measured in one biological replicate at a concentration of $5 \mu \mathrm{M}$ for each PMO-peptide conjugate. The eGFP fluorescence was normalized with respect to the PMO alone condition.

\section{Exon skipping activity of PMO-P6 and PMO-P12 using endocytosis inhibitors}

To interrogate endocytic mechanism, we performed pulse-chase experiment with a panel of endocytosis inhibitors including: chlorpromazine, which is demonstrated to interfere with clathrin-mediated endocytosis; cytochalasin D, which inhibits phagocytosis and macropinocytosis; wortmannin, which alters various endocytosis pathways by inhibiting phosphatidylinositol kinases; EIPA (5-(N-ethyl-N-isopropyl) amiloride), which inhibits macropinocytosis; and Dynasore, which also inhibits clathrinmediated endocytosis. HeLa654 cells were plated at a density of 8,000 cells per well in a 96-well plate in MEM supplemented with $10 \%$ FBS and $1 \%$ penicillin-streptomycin. The stock solutions used for each inhibitor were as follows: chlorpromazine - 10 $\mathrm{mM}$ in water; cytochalasin D - $10 \mathrm{mM}$ in DMSO; wortmannin - $2 \mathrm{mM}$ in DMSO; EIPA - $10 \mathrm{mM}$ in DMSO; Dynasore - $10 \mathrm{mM}$ in DMSO. The next day, the cells were treated with each inhibitor at the following concentrations: chlorpromazine (10 and 1 $\mathrm{mM})$; cytochalasin D (20 and $5 \mathrm{mM})$; wortmannin $(200$ and $50 \mathrm{mM})$; EIPA (50 and $10 \mathrm{mM})$; Dynasore $(80 \mathrm{mM})$ in PBS 1 X. After 30 min, PMO-P6 or PMO-P12 was added to each well at a concentration of $5 \mu \mathrm{M}$. The experiment was conducted in a pulse-chase format, and we evaluated the resulting change in PMO activity. HeLa654 cells were preincubated with various inhibitors for 30 minutes. Treatment with PMO-P6 or PMO-P12 and incubation at $37{ }^{\circ} \mathrm{C}$ and $5 \% \mathrm{CO}_{2}$ for 3 hours was followed by replacement of the media with fresh media (containing neither inhibitor nor PMO-peptide) and the cells were allowed to grow for another 22 hours at $37{ }^{\circ} \mathrm{C}$ and $5 \% \mathrm{CO}_{2}$. Sample preparation and flow cytometry was then performed as usual.

\section{LDH assay}

This assay measures the amount of lactate dehydrogenase (LDH) released into the cell culture supernatant by damaged cells. ${ }^{i}$ Conversion of lactate to pyruvate produces NADH which in turns reduces a yellow tetrazolium salt (iodonitrotetrazolium violet; INT) into a red formazan dye that absorbs at 490 and $492 \mathrm{~nm}$. As a consequence, the amount of LDH in the supernatant is proportional to the amount of formazan and it informs about the number or lysed cells (dead or damaged).

TH1 RPTEC cells were maintained in DMEM-high glucose supplemented with $10 \%$ (v/v) FBS and 1\% (v/v) Pen Strep at $37{ }^{\circ} \mathrm{C}$ and $5 \% \mathrm{CO}_{2}$. Eighteen hours before treatment, TH1 RPTEC cells were plated at a density of 8,000 cells per well in a 96-well plate. The next day, fresh $10 \mathrm{mM}$ stocks of each of PMO-peptide conjugate were prepared in PBS (1X). The concentration of the stocks was determined by measuring the absorbance at $260 \mathrm{~nm}$ and using an extinction coefficient of $168,700 \mathrm{~L} \mathrm{~mol}^{-1} \mathrm{~cm}^{-1}$. The growth media was aspirated from the cells and treatment media was added with each respective conjugate at different concentrations (between 1 and $200 \mu \mathrm{M}$ ) in DMEM-high glucose supplemented with 10\% FBS and 1\% Pen Strep. The cells were incubated with treatment-containing media for 22 hours at $37{ }^{\circ} \mathrm{C}$ and $5 \% \mathrm{CO}_{2}$. Next, the supernatant treatment media was transferred to another clear-bottom 96-well plate for the assay. The assay was performed using the CytoTox 96® Non-Radioactive Cytotoxicity Assay (Promega) according to the included technical bulletin with the only difference of using half of the specified 
amounts ( $25 \mu \mathrm{L}$ of each supernatant, $25 \mu \mathrm{L}$ of the LDH Reagent and $25 \mu \mathrm{L}$ of the stop solution). The absorbance was measured on a BioTek Epoch Microplate Spectrophotometer at $490 \mathrm{~nm}$. The positive and the negative controls correspond to the maximum cell lysis and to the untreated cells respectively. The data were worked up by subtracting the absorbance of untreated cells from all of the treatment conditions, including the cell lysis, and then dividing by the corrected lysis value. The percentage of cytotoxicity was calculated as:

$$
\% \text { Cytotoxicity }=100 \times \frac{(\text { ExperimentalLDHRelease }- \text { MediumBackground })}{(\text { MaximumLDHReleaseControl }- \text { MediumBackground })}
$$

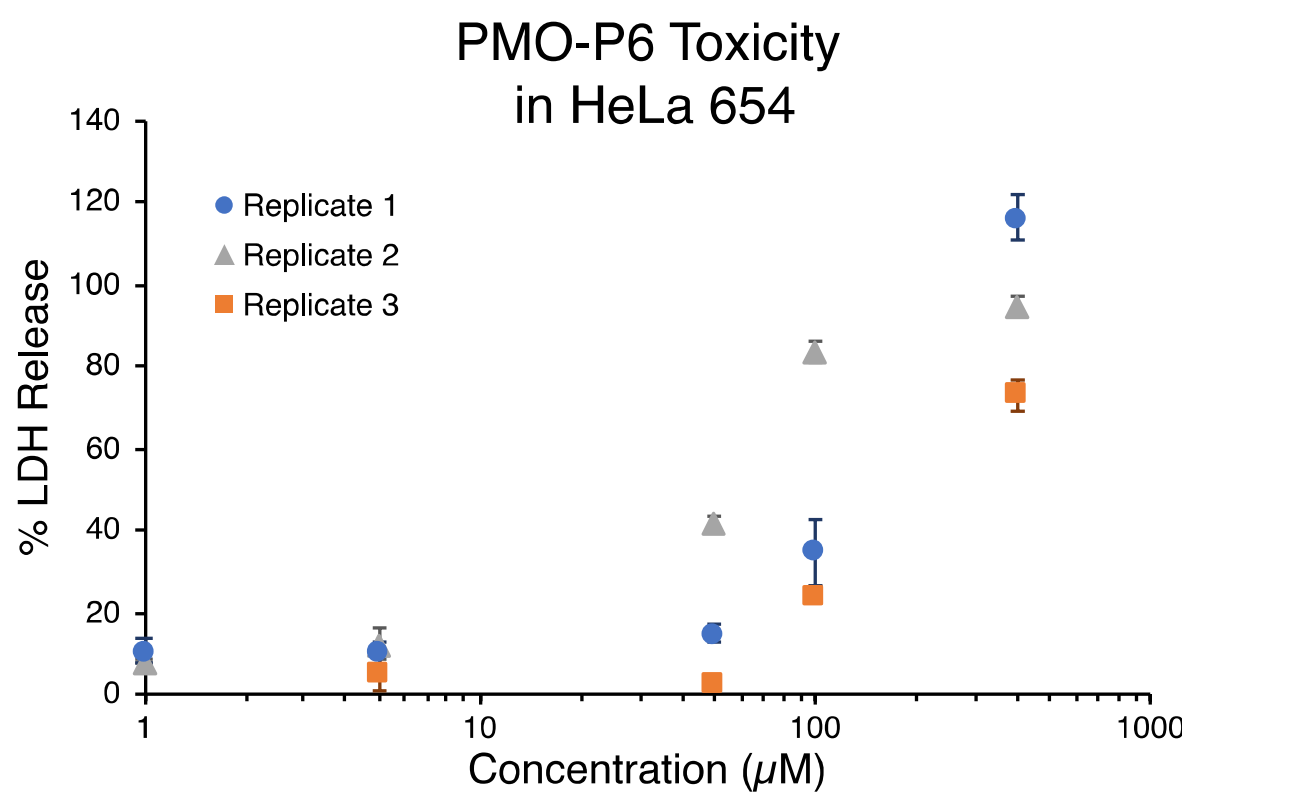

Fig. S9. Dose-response toxicity of PMO-P6 in HeLa 654 cells. Each color corresponds to individual biological replicate.

\section{Synthesis and testing of P6-DTA}

Gly5-DTA was recombinantly expressed and purified via P6-LPSTGG peptide was synthesized and purified by our standard protocol as described above. G5-DTA $(50 \mu \mathrm{M})$ was incubated with P6-DTA $(500 \mu \mathrm{M})$ and $\operatorname{SrtA}^{*}(2.5 \mu \mathrm{M})$ for 90 min at $4{ }^{\circ} \mathrm{C}$ in SrtA buffer $\left(10 \mathrm{mM} \mathrm{CaCl}_{2}, 50 \mathrm{mM}\right.$ Tris, $150 \mathrm{mM} \mathrm{NaCl}, \mathrm{pH}$ 7.5). The reaction was monitored by LCMS and gel electrophoresis. After 90 min, P6-DTA conjugate was isolated using HiTrap SP HP cation exchange column (GE Healthcare, UK) in A: $20 \mathrm{mM}$ Tris, $50 \mathrm{mM} \mathrm{NaCl}, \mathrm{pH} 7.5$ buffer and B: $10 \mathrm{mM}$ Tris, $1 \mathrm{M} \mathrm{NaCl}, \mathrm{pH} 11$, at a gradient of 0-100 over 20 column volumes. Fractions containing the pure product as determined by LCMS and gel electrophoresis were concentrated using a centrifugal filter unit (10K, Millipore). To test for DTA delivery to the cytosol, HeLa cells were plated at 5,000 cells/well in a 96-well plate the day before the experiment. G5-DTA and P6-DTA were prepared at varying concentrations in complete media and transferred to the plate. Cell proliferation was measured after $72 \mathrm{~h}$ using the CellTiter-Glo assay.

\section{Animal studies}

The mice used in the study contain a similar transgene as the HeLa654 cells. EGFP-654 transgenic mice were first obtained from Dr. Ryszard Kole's laboratory. ${ }^{1}$ This mouse model ubiquitously expresses EGFP-654 transgene throughout body under chicken $\beta$-actin promoter. A mutated nucleotide 654 at intron 2 of human $\beta$-globin gene is contained in the EGFP-654 sequence which interrupts EGFP-654 coding sequence and prevents proper translation of EGFP protein. The antisense activity of PMO blocks aberrant splicing and resulted in EGFP expression, the same as in the HeLa 654 assay. In this study, 6- to 8-week-old male EGFP654 mice bred at Charles River Laboratory were shipped to the vivarium at Sarepta Therapeutics (Cambridge, MA). These mice were group housed with ad libitum access to food and water. All animal protocols were approved by the Institutional Animal Care and Use Committee (IACUC) of Sarepta Therapeutics. 
Before injection, the PMO-peptide was confirmed to have minimal endotoxin levels. For the endotoxin assay measurement using the PMO-P6 lot that was used for animal studies, $0.5 \mathrm{mg}$ of PMO-P6 as acetate salt were dissolved in $1 \mathrm{~mL}$ of PBS (1X). The cartridge used was the 0.01 of the Charles River Endosafe nexgen-PTS. $25 \mu \mathrm{L}$ of the sample were placed into each of the four sample reservoirs of the cartridge. The lot of PMO-P6 (63 mg as acetate salt) used for animal studies showed 0.0471 EU/mg (EU refers to Endotoxin Units).

After 3-days of acclimation, mice were randomized into groups to receive a single i.v. tail vein injection of either saline or PMO$\mathbf{P 6}$ at the indicated doses; $0,10,30$, and $60 \mathrm{mg} / \mathrm{kg}$. Seven days after the injection, the mice were euthanized for serum and tissue sample collection. Quadriceps, diaphragm, heart were rapidly dissected, snap-frozen in liquid nitrogen and stored at $-80{ }^{\circ} \mathrm{C}$ until analysis.

Serum from all groups were collected seven days post-injection and tested for kidney injury markers using a Vet Axcel Clinical Chemistry System (Alfa Wassermann Diagnostic Technologies, LLC). Specifically, serum BUN, creatinine, and cystatin C levels were measured using ACE® Creatinine Reagent (Alfa Wassermann, Cat\# SA1012), ACE® Blood Urea Nitrogen Reagent (Alfa Wassermann, Cat\# SA2024) and Diazyme Cystatin C immunoassay (Diazyme Laboratories, Cat\# DX133C-K), respectively, per manufacturer's recommendation.

20-25 mg of mouse tissue was homogenized in RIPA buffer (Thermo Fisher, Cat\# 89900) with protease inhibitor cocktail (Roche, 04693124001) using a Fast Prep 24-5G instrument (MP Biomedical). Homogenates were centrifuged at 12,000 g for 10 min at 4 ${ }^{\circ} \mathrm{C}$. The resultant supernatant lysates were quantified by Pierce BCA Protein Assay Kit (Thermo Fisher, Cat\# 23225) and saved for EGFP expression measurement. Specifically, $80 \mu \mathrm{g}$ of lysates were aliquoted in each well in a black-wall clear-bottom 96well microplate (Corning). EGFP fluorescent intensity of each sample was measured in duplicates using a SpectraMAx i3x microplate reader (Molecular devices) by default setting. The average EGFP fluorescent intensity of each sample was then plotted against a standard curve constructed by recombinant EGFP protein (Origen, Cat\#TP790050) to quantify EGFP protein level per $\mu \mathrm{g}$ protein lysate.

\section{Machine Learning Model}

We trained a series of machine learning models with different data augmentation. In addition to the manuscript text, we have noted some observations here. To find the best model architecture for activity prediction, we tested 11 different model architectures, from simpler models, such as lasso and random forest regressor, to convolutional neural networks, with both $1 \mathrm{x}$ and 10x data augmentation. The sequences were featurized using matrices of monomer fingerprints as previously described. For the training, we split the dataset into $60 \%$ training, $20 \%$ validation to update the weights during the training, and held out $20 \%$ of the dataset to evaluate the model post-training. ${ }^{2}$ For robust predictions, we performed model ensembling, with a total of 25 models for each model architecture (5 random weight initializations x top 5 hyperparameter sets).

The predicted peptides were validated experimentally, and their experimental activities were compared to the model's predicted activity to judge the prediction accuracy. We noted that the CNN model indeed performed the best amongst all other models on the dataset of sequences that were experimentally tested with 0.148 R2 score and 0.512 Pearson's correlation. For uRMSE on the experiment dataset, we noted that simpler models had slightly better values than CNN models, but the error bars in the parity plots were significantly large, thereby rendering the mean uRMSE values unreliable.

10x augmented convolutional neural network (CNN) and 1x multi-layer perceptron (MLP) models performed the best amongst all augmentation and model architectures. On the test dataset, 1x MLP outperformed all models on unitless root mean squared error (uRMSE, RMSE scaled to standard deviation 1) and Pearson's correlation metrics, while 10x CNN had the highest R2 value. Given the better extrapolation ability of CNN as compared to MLP, we hypothesized that CNN would be a better choice for generation of new sequences.

Data augmentation results in worse performance of simpler models, while improving the performance of CNN models (Table S2, Figure S2). We noted that simpler model architectures such as Gaussian process and support vector regression overfit on the training data, and performed poorly on the test and experiment datasets, as noted from the tabulated metrics and significantly large error bars in the parity plots. This behavior may be attributed to the method of training, where the simpler models are trained in one-shot by showing all the data in the training set including the augmented sequences which bias the error correction during training. On the other hand, CNN models are trained by showing data in smaller batches. We hypothesize that the batched training 
results in weights of different layers being constrained to be updated to predict for the desired design space as they see similar datapoints across all batches.

For the CNN models, we tested different data augmentations ranging from $1 \mathrm{x}$ to $20 \mathrm{x}$, and note that $10 \mathrm{x}$ was the optimal augmentation on the basis of balancing the distribution of sequences of different lengths in the training dataset and performance on the held-out test dataset (Table S3). With increasing augmentation of sequences with 20 residues or less, we noted that overfitting caused the metrics in the validation dataset to improve. On the held-out test dataset, uRMSE worsened with augmentation, from 0.73 of the standard deviation of training data for $1 \mathrm{x}$, to 0.75 for $10 \mathrm{x}, 0.84$ for $15 \mathrm{x}$, and 0.89 for $20 \mathrm{x}$ CNN models. For $\mathrm{R}^{2}$ score and Pearson's correlation metrics, $10 \mathrm{x}$ CNN was seen to be the best model. As performance on the test dataset demarcates the performance of the model for sequences of all lengths and denotes robust training, we chose 10x CNN model for further usage. The model choice was validated by metrics noted in the experiment dataset, where the 10x CNN model was a close second for uRMSE and Pearson's correlation, and had the highest $\mathrm{R}^{2}$ score amongst other CNN models.

Table S1. Metrics for validation, test and experiment datasets for simpler models trained with 1x augmentation, and CNN models with $1 \mathrm{x}$ and $10 \mathrm{x}$ augmentation. ${ }^{\mathrm{a}}$

\begin{tabular}{|c|c|c|c|c|c|c|c|c|c|c|c|c|}
\hline \multirow{2}{*}{ Model } & \multicolumn{4}{|c|}{ Validation } & \multicolumn{4}{|c|}{ Test } & \multicolumn{4}{|c|}{ Experiment } \\
\hline & URMSE & R2 & P C & SC & uRMSE & $\mathbf{R 2}$ & P C & SC & uRMSE & $\mathbf{R 2}$ & P C & SC \\
\hline CNN-1x & 0.572 & 0.669 & 0.835 & 0.865 & 0.728 & 0.533 & 0.747 & 0.806 & 1.127 & -0.226 & 0.486 & 0.490 \\
\hline CNN-10x & 0.378 & 0.853 & 0.927 & 0.867 & 0.745 & 0.690 & 0.832 & 0.835 & 1.395 & 0.148 & 0.512 & 0.577 \\
\hline GP & 0.099 & 0.990 & 0.995 & 0.989 & 0.672 & -0.141 & 0.809 & 0.796 & 0.932 & -9.607 & 0.502 & 0.606 \\
\hline $\begin{array}{l}\text { Gradient } \\
\text { Boosting }\end{array}$ & 0.125 & 0.983 & 0.993 & 0.960 & 0.629 & 0.433 & 0.809 & 0.842 & 1.116 & -25.518 & 0.336 & 0.315 \\
\hline KNN & 0.341 & 0.842 & 0.943 & 0.908 & 0.672 & 0.263 & 0.779 & 0.851 & 1.404 & -66.896 & 0.356 & 0.461 \\
\hline Lasso & 0.684 & -2.384 & 0.893 & 0.844 & 0.838 & -4.971 & 0.759 & 0.812 & 1.047 & -20.082 & 0.315 & 0.451 \\
\hline MLP & 0.168 & 0.967 & 0.987 & 0.924 & 0.580 & 0.505 & 0.842 & 0.845 & 0.958 & -6.926 & 0.410 & 0.535 \\
\hline $\begin{array}{c}\text { Random } \\
\text { Forest }\end{array}$ & 0.222 & 0.937 & 0.979 & 0.912 & 0.692 & 0.222 & 0.762 & 0.862 & 1.098 & -11.305 & 0.077 & 0.210 \\
\hline Ridge & 0.305 & 0.882 & 0.954 & 0.860 & 0.666 & 0.220 & 0.786 & 0.781 & 1.046 & -2.912 & 0.353 & 0.599 \\
\hline SGD & 0.869 & -29.192 & 0.832 & 0.825 & 0.970 & -45.410 & 0.733 & 0.831 & 1.265 & -1106 & 0.331 & 0.551 \\
\hline SVR & 0.278 & 0.868 & 0.985 & 0.915 & 0.756 & -1.383 & 0.775 & 0.770 & 0.928 & -6.169 & 0.429 & 0.600 \\
\hline XGBoost & 0.365 & 0.736 & 0.965 & 0.884 & 0.734 & -0.374 & 0.743 & 0.822 & 1.055 & -9.107 & 0.244 & 0.327 \\
\hline
\end{tabular}

${ }^{a}$ Abbreviations: GP - Gaussian Process, KNN - k-nearest neighbors, MLP - multi-layer perceptron, SGD - stochastic gradient descent, SVR - support vector regression, XGBoost - extreme gradient boosting. PC - Pearson's correlation coefficient, SC - Spearman's rank correlation coefficient. 

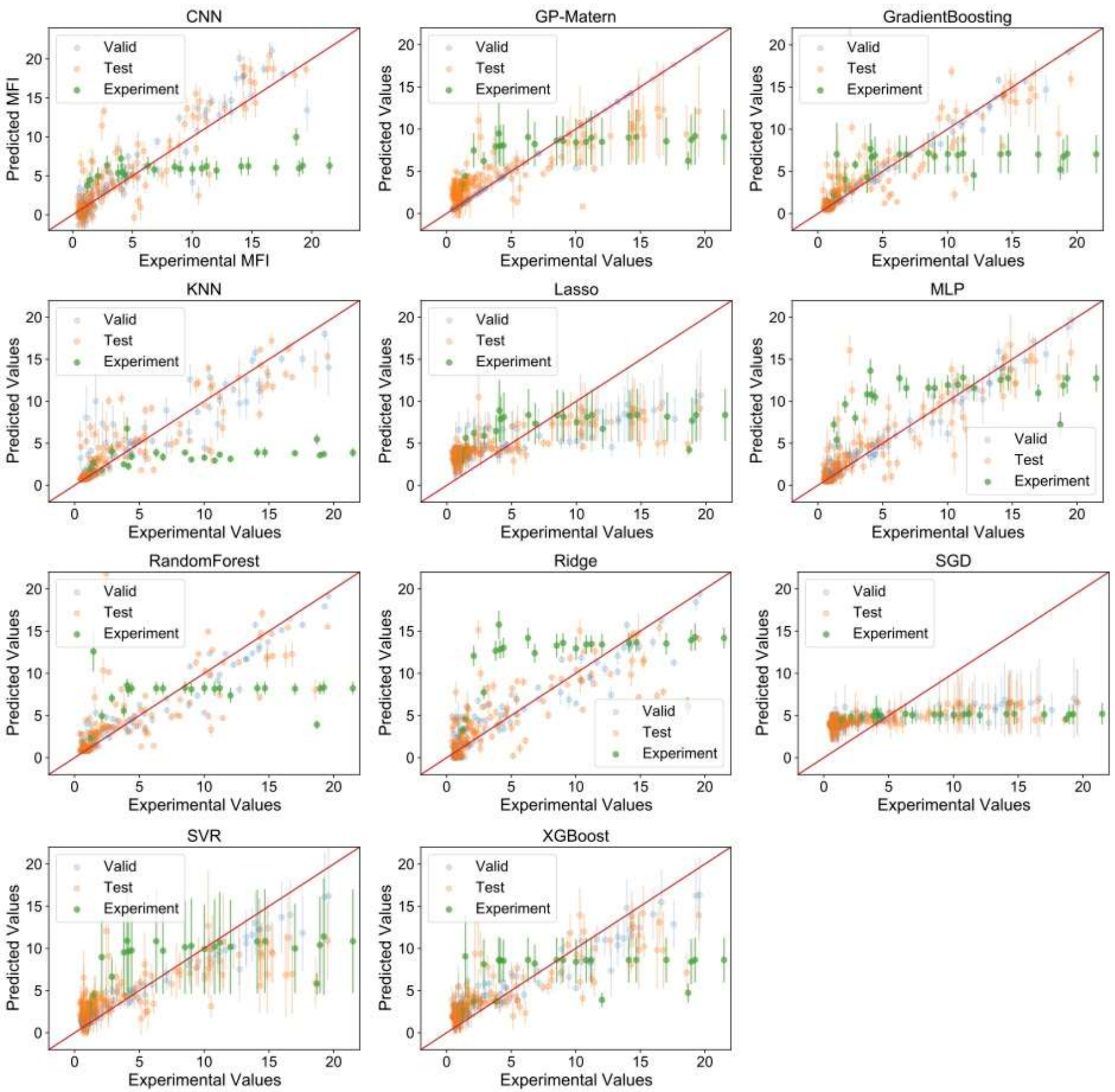

Fig. S10. Parity plots with predicted and experimental values for validation, test and experiment datasets for simpler models and CNN models with 1x. Datapoints belonging to the validation, test and experiment sets are in different colors, as noted in the legend.

The model architecture corresponding to each subplot has been noted in the title. The points indicate the mean value of the predicted MFI from all the models in the ensemble, and the error bars denote the standard deviation. Abbreviations: GPMatern - Gaussian Process with Matern kernel, KNN - k-nearest neighbors, MLP - multi-layer perceptron, SGD - stochastic gradient descent, SVR - support vector regression, XGBoost - extreme gradient boosting. 
Table S2. Metrics for validation, test and experiment datasets for simpler models trained with 10x augmentation, and CNN models with $1 \mathrm{x}$ and $10 \mathrm{x}$ augmentation. ${ }^{\mathrm{a}}$

\begin{tabular}{|c|c|c|c|c|c|c|c|c|c|c|c|c|}
\hline \multirow{2}{*}{ Model } & \multicolumn{4}{|c|}{ Validation } & \multicolumn{4}{|c|}{ Test } & \multicolumn{4}{|c|}{ Experiment } \\
\hline & uRMSE & $\mathbf{R 2}$ & P C & SC & uRMSE & $\mathbf{R 2}$ & P C & SC & uRMSE & $\mathbf{R 2}$ & P C & S C \\
\hline CNN-1x & 0.572 & 0.669 & 0.835 & 0.865 & 0.728 & 0.533 & 0.747 & 0.806 & 1.127 & -0.226 & 0.486 & 0.490 \\
\hline CNN-10x & 0.378 & 0.853 & 0.927 & 0.867 & 0.745 & 0.690 & 0.832 & 0.835 & 1.395 & 0.148 & 0.512 & 0.577 \\
\hline GP & 0.094 & 0.991 & 0.995 & 0.987 & 0.814 & 0.075 & 0.816 & 0.812 & 1.184 & -9.469 & 0.479 & 0.592 \\
\hline $\begin{array}{l}\text { Gradient } \\
\text { Boosting }\end{array}$ & 0.114 & 0.986 & 0.994 & 0.987 & 0.806 & 0.398 & 0.799 & 0.846 & 1.298 & -16.597 & 0.406 & 0.517 \\
\hline KNN & 0.249 & 0.913 & 0.975 & 0.963 & 0.850 & 0.310 & 0.773 & 0.850 & 1.894 & -18.284 & 0.105 & 0.155 \\
\hline Lasso & 0.721 & -4.624 & 0.908 & 0.857 & 1.072 & -6.721 & 0.775 & 0.810 & 1.418 & -43.690 & 0.411 & 0.512 \\
\hline MLP & 0.096 & 0.990 & 0.995 & 0.982 & 0.730 & 0.562 & 0.838 & 0.807 & 1.211 & -4.440 & 0.488 & 0.528 \\
\hline $\begin{array}{c}\text { Random } \\
\text { Forest }\end{array}$ & 0.282 & 0.877 & 0.972 & 0.952 & 0.883 & 0.092 & 0.755 & 0.852 & 1.295 & -8.578 & 0.466 & 0.456 \\
\hline Ridge & 0.265 & 0.912 & 0.965 & 0.884 & 0.839 & 0.215 & 0.783 & 0.778 & 1.216 & -3.298 & 0.381 & 0.591 \\
\hline SGD & 0.886 & -49.522 & 0.823 & 0.907 & 1.243 & -59.090 & 0.704 & 0.827 & 1.645 & -1434 & 0.487 & 0.610 \\
\hline SVR & 0.286 & 0.848 & 0.988 & 0.950 & 0.957 & -1.472 & 0.769 & 0.772 & 1.154 & -5.489 & 0.444 & 0.598 \\
\hline XGBoost & 0.356 & 0.733 & 0.971 & 0.934 & 0.931 & -0.323 & 0.730 & 0.835 & 1.278 & -7.528 & 0.285 & 0.250 \\
\hline
\end{tabular}

${ }^{a}$ Abbreviations: GP - Gaussian Process, KNN - k-nearest neighbors, MLP - multi-layer perceptron, SGD - stochastic gradient descent, SVR - support vector regression, XGBoost - extreme gradient boosting. PC - Pearson's correlation coefficient, SC - Spearman's rank correlation coefficient. 

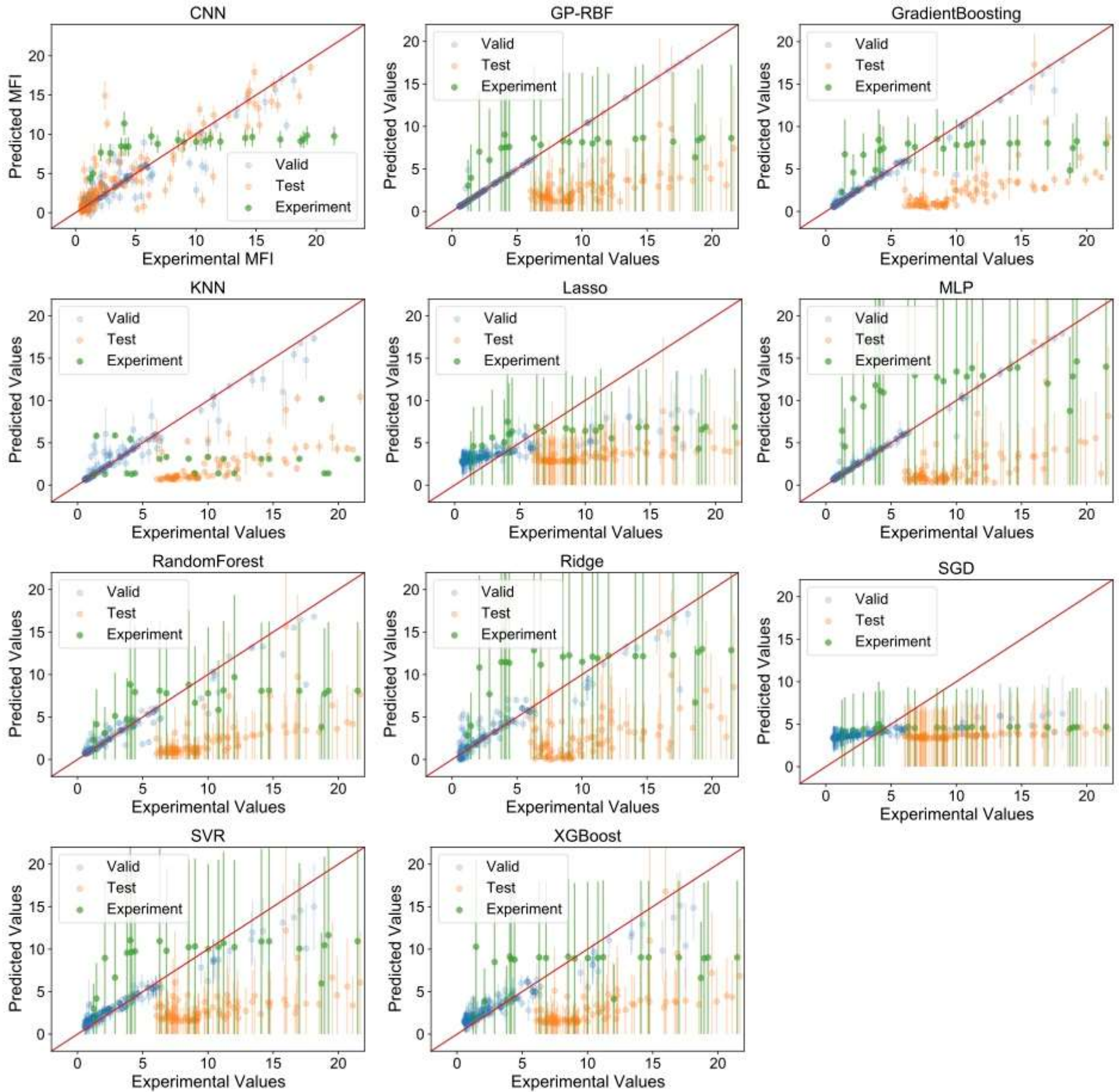

Fig. S11. Parity plots with predicted and experimental values for validation, test and experiment datasets for simpler models and CNN models with 10x augmentation.

The model architecture corresponding to each subplot has been noted in the title. The points indicate the mean value of the predicted MFI from all the models in the ensemble, and the error bars denote the standard deviation. Abbreviations: GPRBF - Gaussian Process with radial basis function kernel, KNN - k-nearest neighbors, MLP - multi-layer perceptron, SGD - stochastic gradient descent, SVR - support vector regression, XGBoost - extreme gradient boosting. 
Table S3. Metrics for validation, test and experiment datasets for CNN models with 1x, 10x 15x and 20x augmentation. The top 2 metrics for each column are noted in red and blue, respectively. 10x augmentation performs really well for test and experiment datasets. This augmentation is also noted to be the most optimal choice based on the overfitting on the train and valid datasets for 20x augmentation. PC - Pearson's correlation coefficient, SC - Spearman's rank correlation coefficient.

\begin{tabular}{|c|c|c|c|c|c|c|c|c|c|c|c|c|}
\hline \multirow{2}{*}{ Augment } & \multicolumn{4}{|c|}{ Valid } & \multicolumn{4}{|c|}{ Test } & \multicolumn{4}{|c|}{ Experiment } \\
\hline & uRMSE & $\mathbf{R 2}$ & PC & SC & uRMSE & $\mathbf{R 2}$ & PC & SC & uRMSE & $\mathbf{R 2}$ & PC & sc \\
\hline $1 x$ & 0.572 & 0.669 & 0.835 & 0.865 & 0.728 & 0.533 & 0.747 & 0.806 & 1.127 & -0.226 & 0.486 & 0.490 \\
\hline $10 x$ & 0.378 & 0.853 & 0.927 & 0.867 & 0.745 & 0.690 & 0.832 & 0.835 & 1.395 & 0.148 & 0.512 & 0.577 \\
\hline $15 x$ & 0.418 & 0.867 & 0.935 & 0.883 & 0.837 & 0.673 & 0.823 & 0.833 & 1.739 & 0.111 & 0.499 & 0.555 \\
\hline $20 x$ & 0.323 & 0.896 & 0.948 & 0.871 & 0.888 & 0.681 & 0.827 & 0.830 & 1.983 & 0.121 & 0.562 & 0.581 \\
\hline
\end{tabular}


A
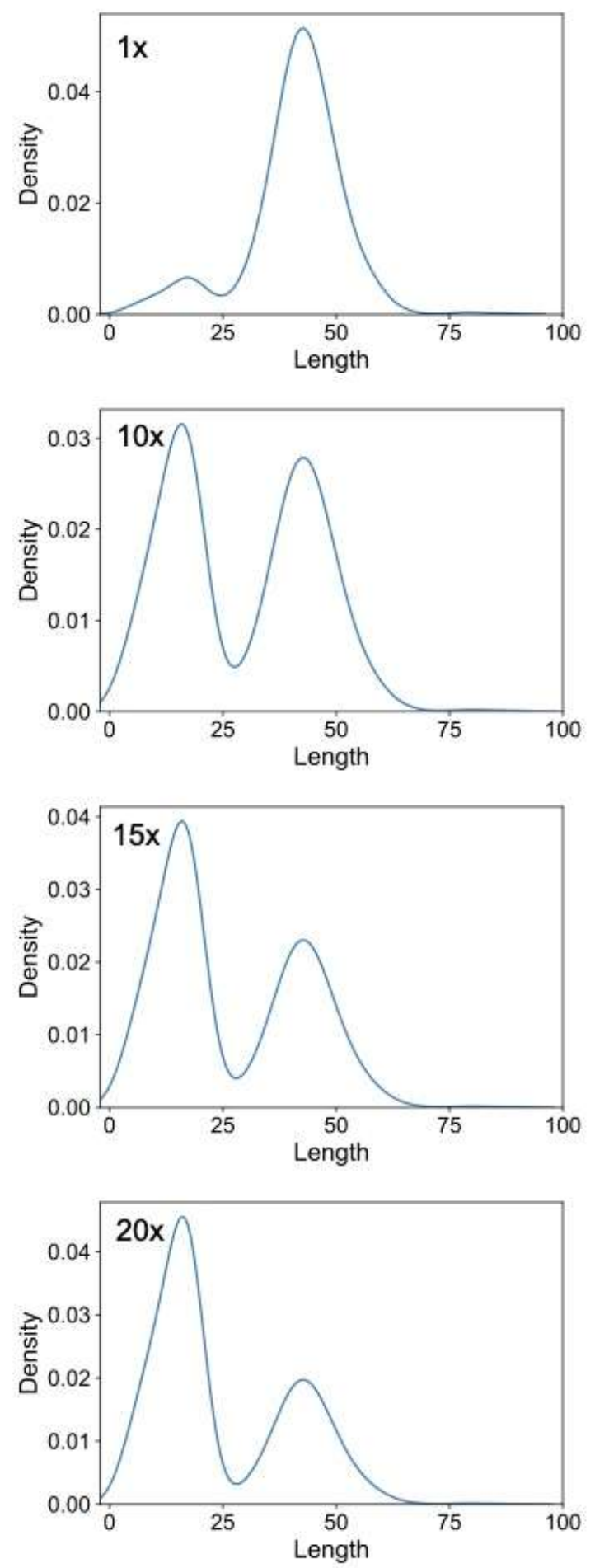

B Valid Test Experiment
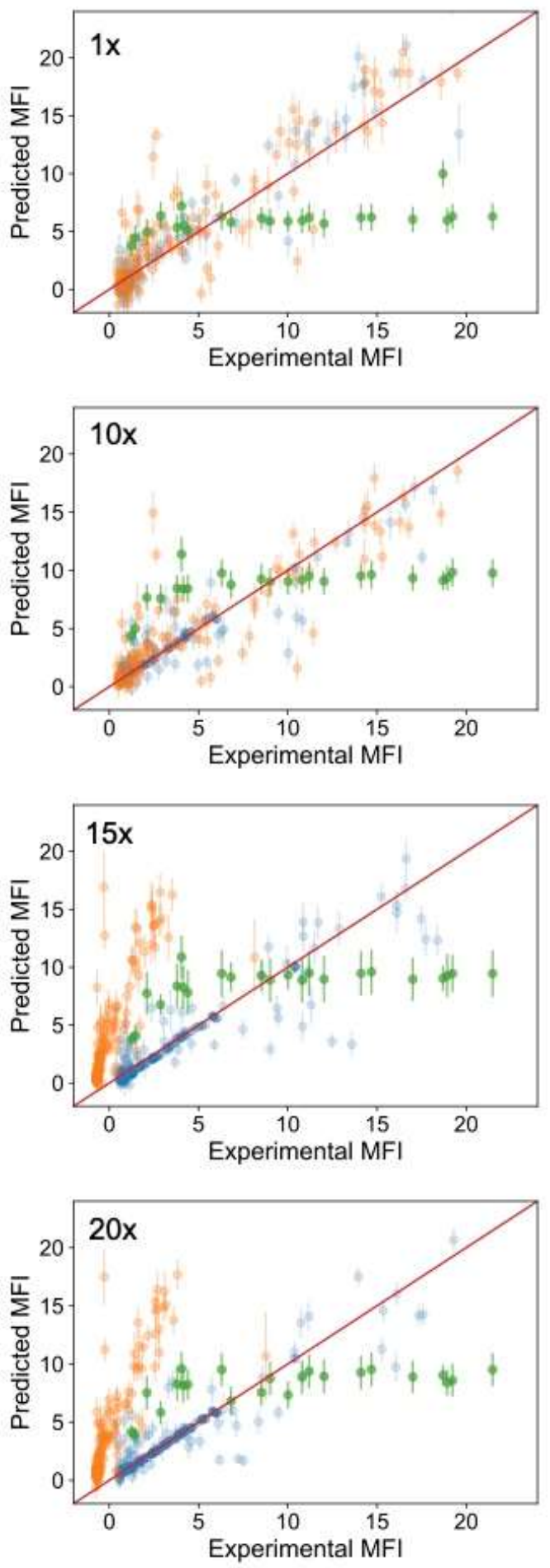

Fig. S12. Performance of CNN models trained with different data augmentation. A) The distribution of lengths of sequences for training datasets with different data augmentations. B) Parity plots with predicted and experimental MFI values for validation, test and experiment datasets for $\mathrm{CNN}$ models with different data augmentation. The data augmentation has been noted in the respective plots. Datapoints belonging to the valid, test and experiment sets are in different colors, as noted in the legend. 
We evaluated retrospectively how active learning would impact prediction accuracy for the new sequences. To start with, we had 653 sequences in the training dataset and 19 sequences, with 20 residues or less, which were experimentally validated. For the first generation of active learning models, we added 1 sequence to the training data set and tested on 18 . Subsequently, in each generation, we added 3 sequences, with $(4,15),(7,12)$, and ultimately $(16,3)$ in the (train, test) data sets. For each generation, we used the top 5 model architectures from the 10x augmentation run, and followed a similar training methodology in terms of hyperparameters and random seeds for weight initialization. Given that we had an external test set, we trained with 80:20 train:validation set. To simulate a real-world scenario, all sequences from the previous generation were carried on to the next generation, and 3 random sequences were added to the training data set in each generation. We performed 3 such active learning simulations, varying the order in which the sequences are added to the train set, and averaged the results across all of them.

The test set performance improved every time new sequences were added to the training data set. However, the performance on the validation data set worsened slightly towards the end, owing to significantly more short CPP sequences than longer sequences, similar to results observed for 20x augmentation. The improving model accuracy on the test dat set indicates that active learning with new sequences can help in sequential optimization of the model to predict sequences in desired design space (Fig S13).

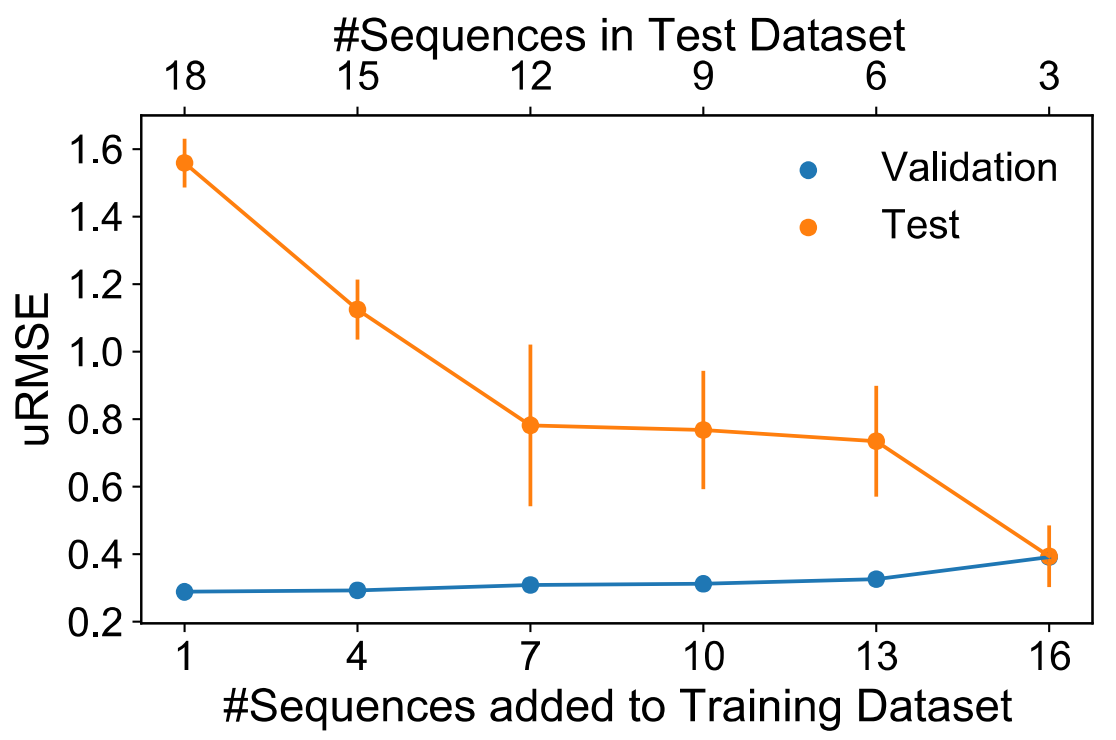

Fig S13. Model performance on the held out test dataset measured as uRMSE of short CPP sequences improves as we add more short sequences to the training dataset. 


\section{Evaluation of C-terminal cysteine residue}

To further examine the role of the C-terminal cysteine residue, we made several analogs of P6. First, we made a dimeric form to mimic disulfide formation by conjugating two copies of $\mathrm{P} 6$ through a $\mathrm{S}_{\mathrm{N}} \mathrm{Ar}$ reaction using decafluorobiphenyl (Fig. S14A). Synthesis of the deca-linked P6 dimer was carried out as previously described. ${ }^{3}$

Next, we mutated the C-terminal cysteine residue to a methionine (P20) or deleted the residue altogether (P21) and compared the activities to the $\mathrm{C} 18 \mathrm{~A}$ mutant, $\mathrm{P} 8$. These analogs were then evaluated using the HeLa 654 assay and their activities were normalized to PMO alone.

We found that the activity of the P6 dimer was similar to that of P6, whereas the activities of P20 and P21 were similar to that of P8. This suggests that it is possible that the disulfide dimer is the active form of P6 once inside cells, although thiolmediated uptake could still be playing a role as well. Clearly the analogs which lack the C-terminal cysteine exhibit a decrease, but not complete ablation, of delivery activity. Therefore, the thiol residue is necessary but not sufficient for the observed activity.

A)

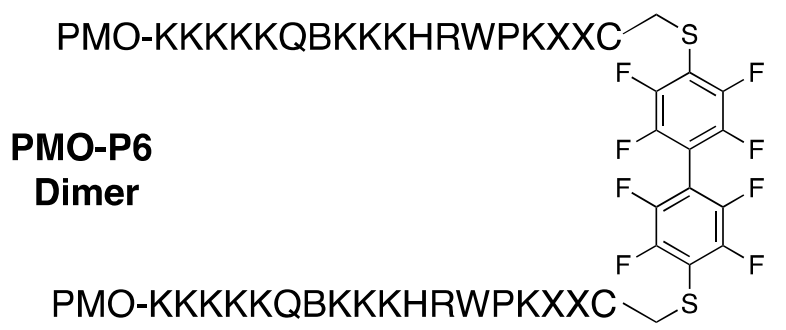

B)

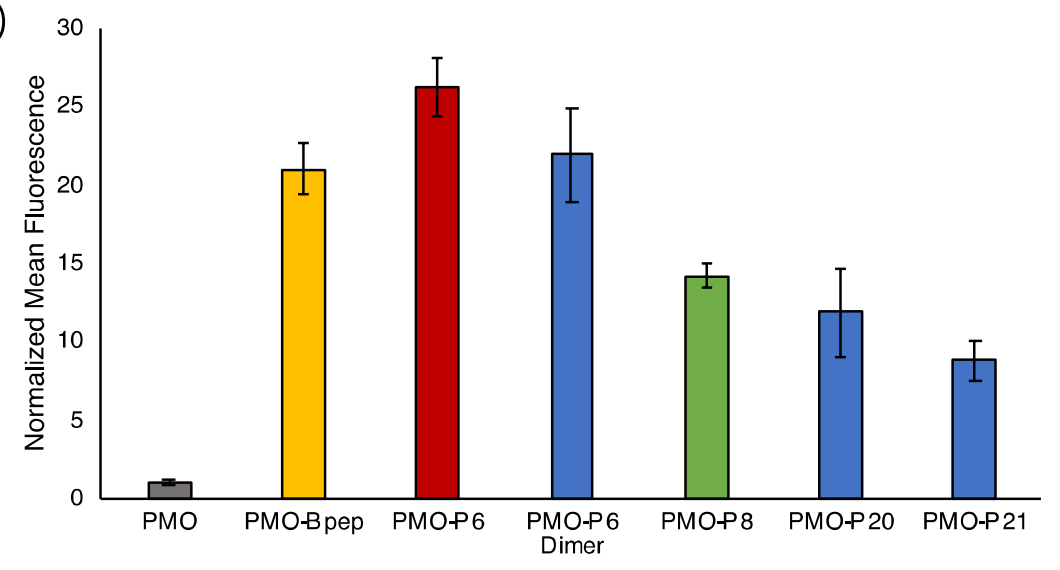

C)

\begin{tabular}{|c|c|c|c|}
\hline Peptide & Sequence & Net Charge & Activity \\
\hline PMO-Bpep & RXRRBRRXRRBR & 8 & 24 \\
\hline PMO-P6 & KKKKKQBKKKHRWPKXXC & 11 & 26 \\
\hline PMO-P6 Dimer & KKKKKQBKKKHRWPKXXC-(deca)-CXXKPWRHKKKBQKKKK & 22 & 22 \\
\hline PMO-P8 & KKKKKQBKKKHRWPKXXA & 11 & 14 \\
\hline PMO-P20 & KKKKKQBKKKHRWPKXXM & 11 & 12 \\
\hline PMO-P21 & KKKKKQBKKKHRWPKXX & 11 & 9 \\
\hline
\end{tabular}

Fig S14. Evaluation of role of C-terminal cysteine residue. (A) Structure of PMO-P6 dimer, where C-terminal cysteine residues are connected via decafluorobiphenyl. (B) Shown are activities of various mutated sequences as determined by EGFP assay and reported as fluorescence relative to PMO alone. (C) Table of sequences corresponding to activities shown in $\mathrm{B}$ ) as well as net charge of each sequence and activity relative to PMO alone. Bars represent group mean $\pm \mathrm{SD}, \mathrm{N}=3$. 


\section{Appendix I: LC-MS characterization}

azide-P1

Mass expected: $725.5 \mathrm{Da}$

Mass observed: 725.4 Da

Peptide sequence: KXKXT
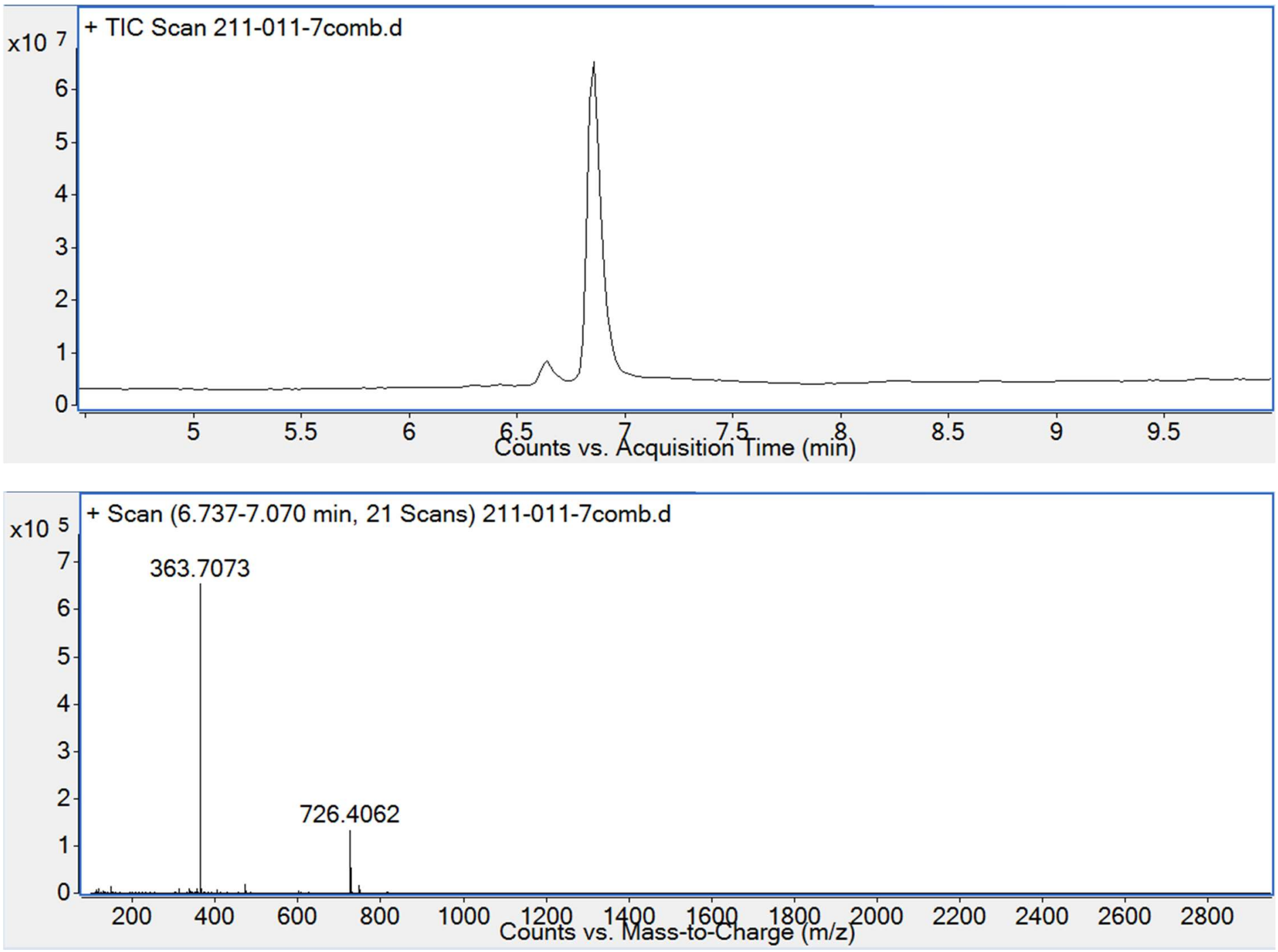
azide-P2

Mass expected: $1273.8 \mathrm{Da}$

Mass observed: 1273.6 Da

Peptide sequence: KXKHQQQXK
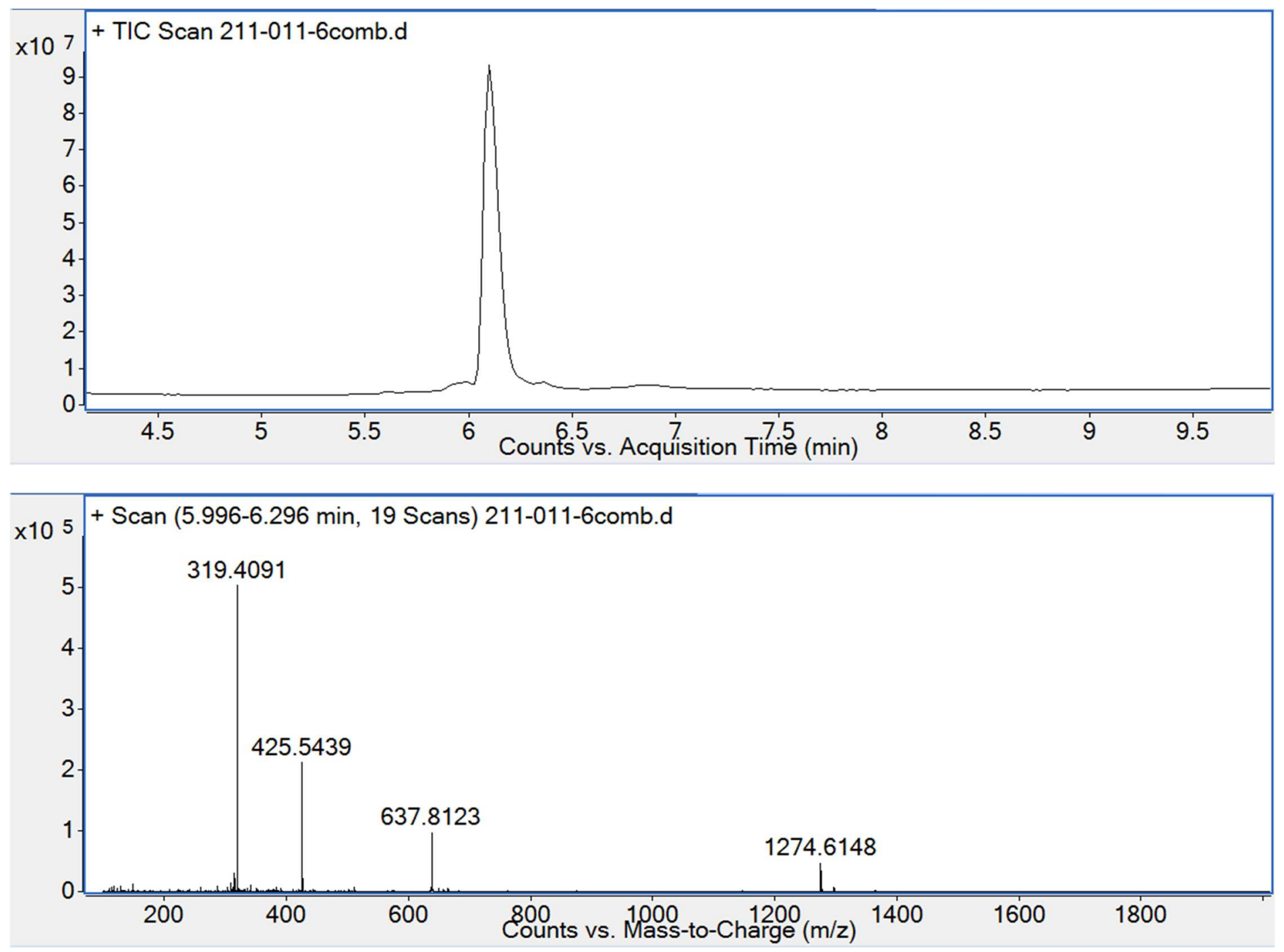
azide-P3

Mass expected: $1630.0 \mathrm{Da}$

Mass observed: 1629.9 Da

Peptide sequence: KXKKQQGKKKHR
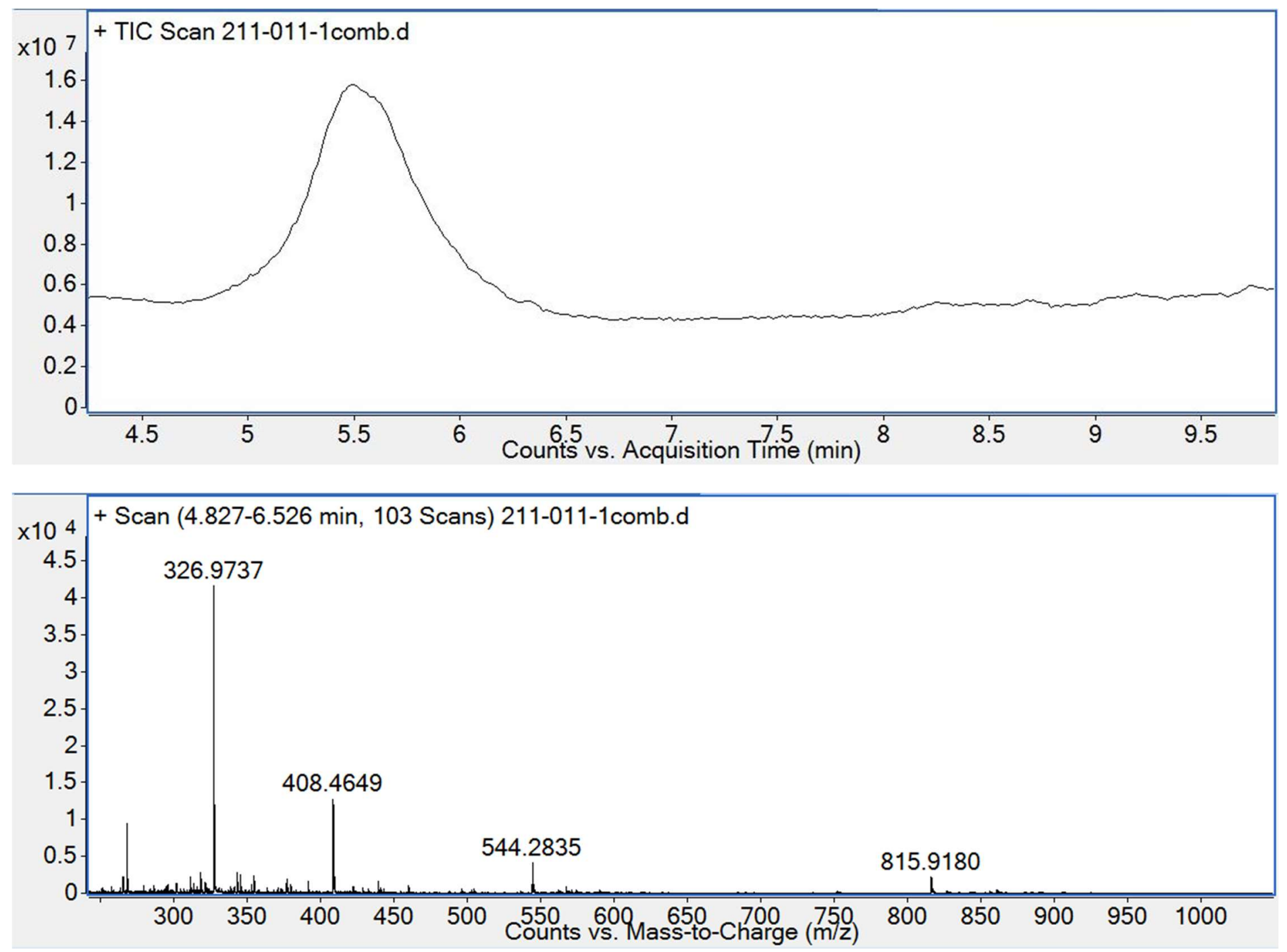
azide-P4

Mass expected: $1951.2 \mathrm{Da}$

Mass observed: 1952.0 Da

Peptide sequence: HKKKKQBKKKHRWP
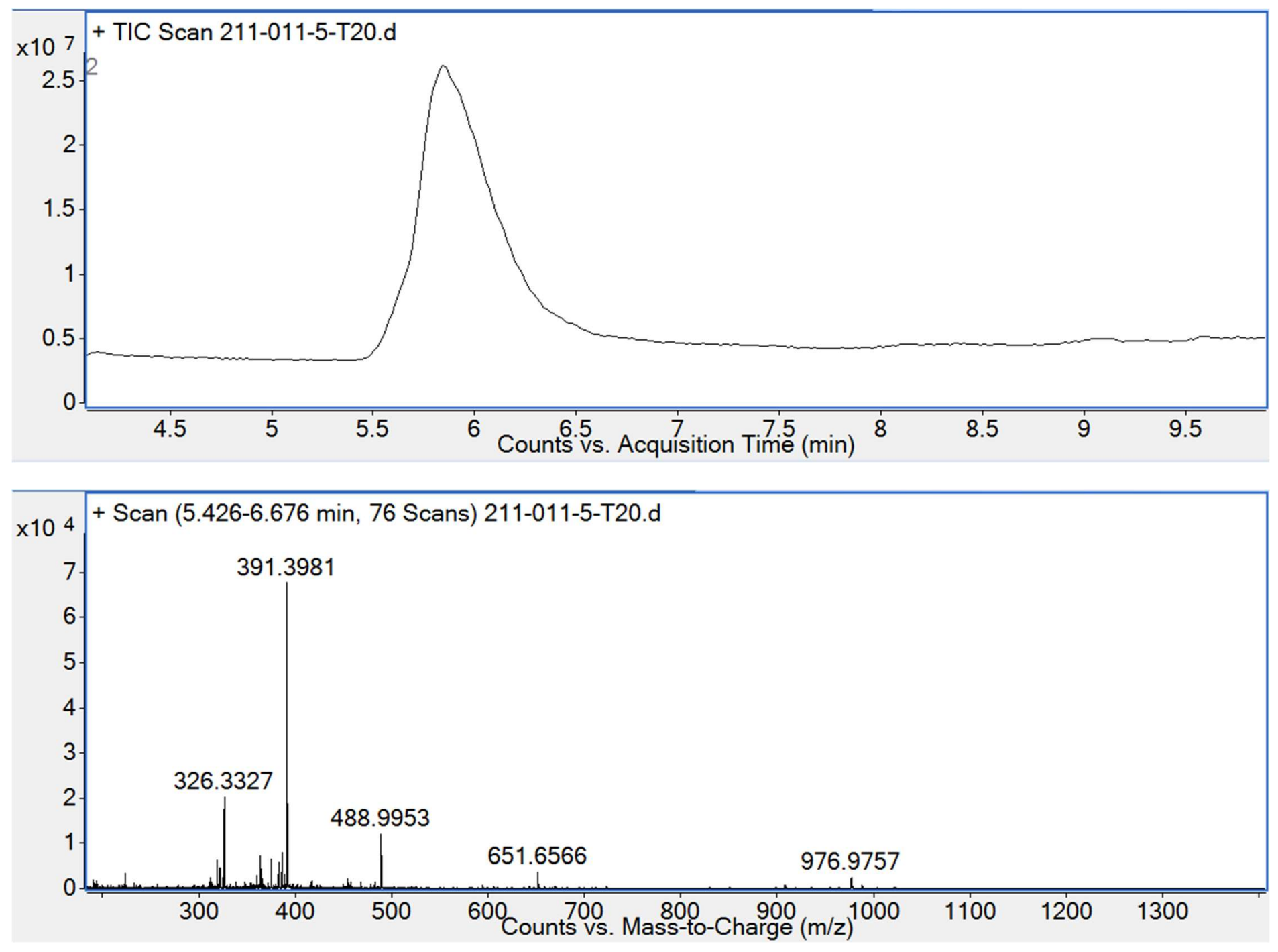
azide-P5

Mass expected: $2131.3 \mathrm{Da}$

Mass observed: 2131.1 Da

Peptide sequence: KKKKKQBKKKHRWPMG
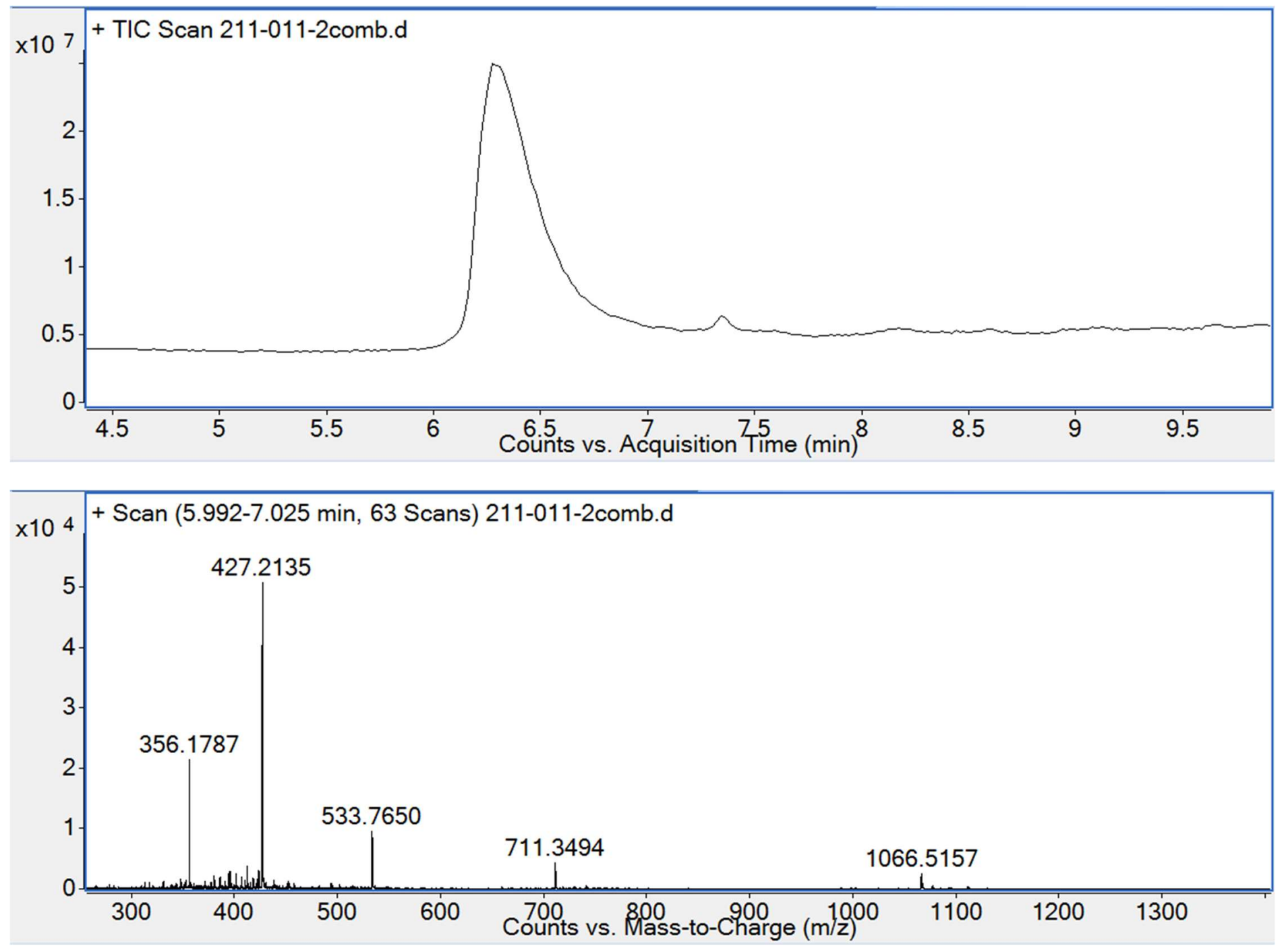


\section{azide-P6}

Mass expected: $2400.5 \mathrm{Da}$

Mass observed: $2400.2 \mathrm{Da}$

Peptide sequence: KKKKKQBKKKHRWPKXXC
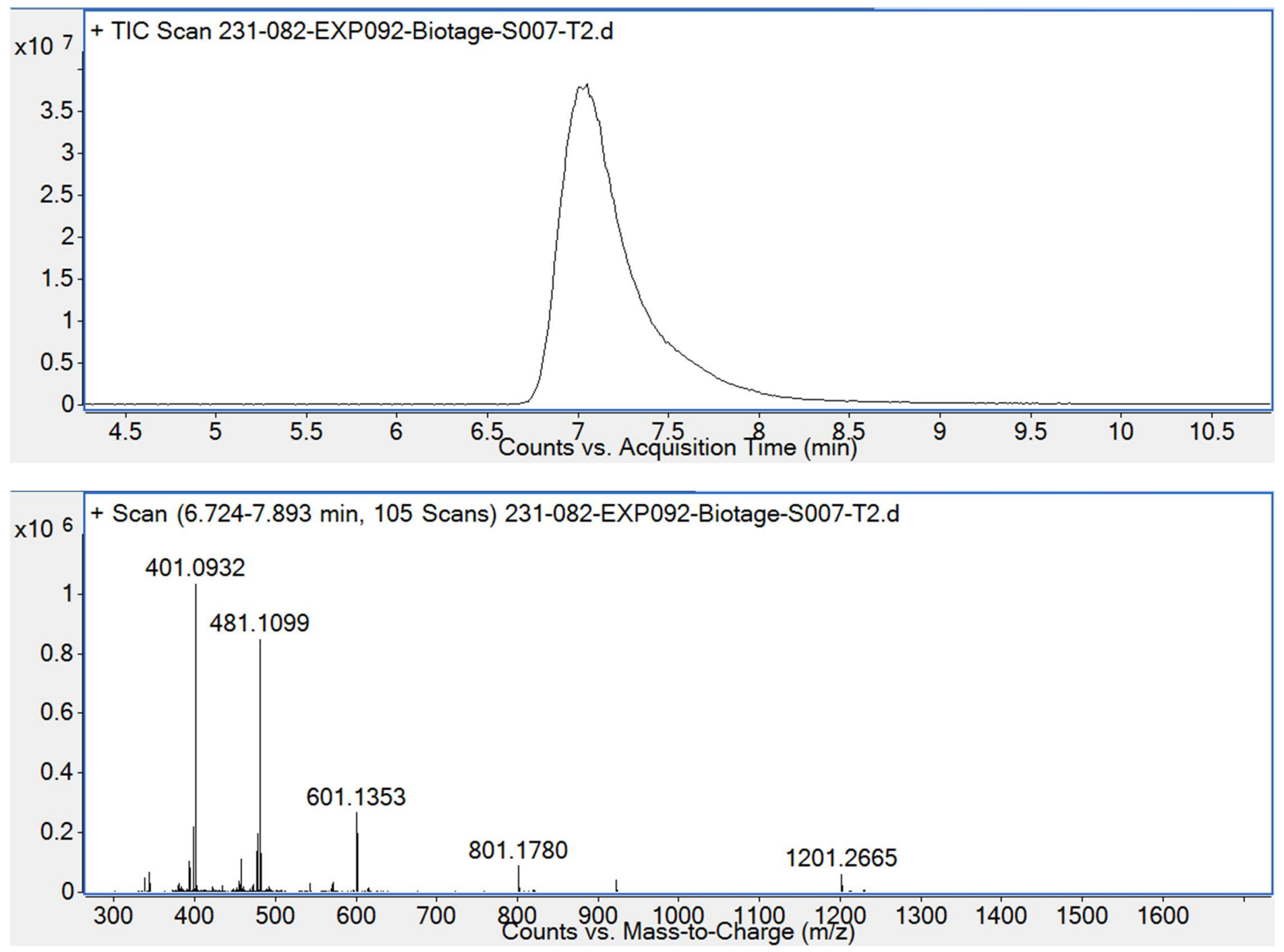
azide-P7

Mass expected: $2629.5 \mathrm{Da}$

Mass observed: 2629.2 Da

Peptide sequence: KKKKNQBKKKHRWPMKXCPQ
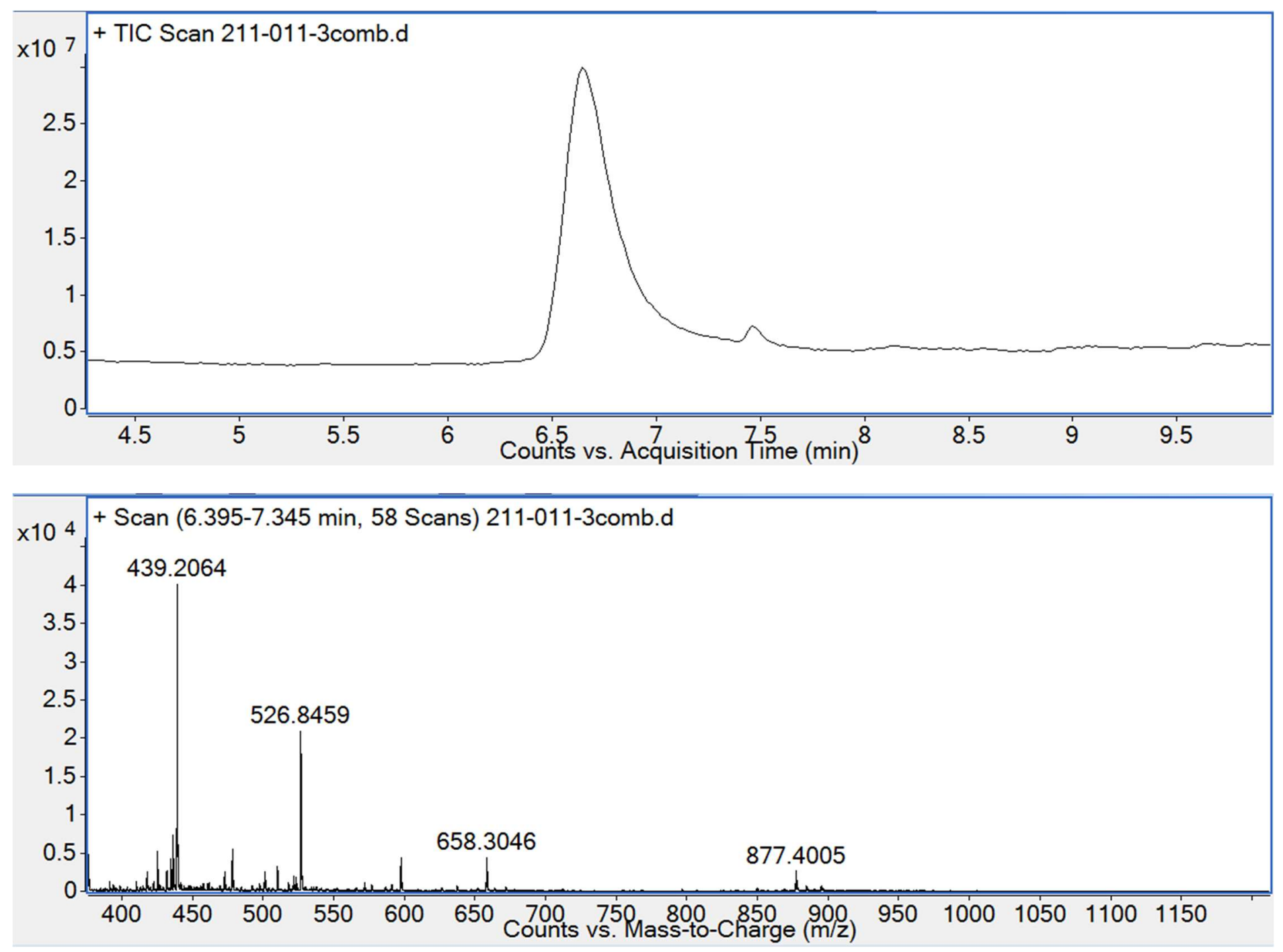


\section{azide-P8}

Mass expected: $2367.5 \mathrm{Da}$

Mass observed: $2368.6 \mathrm{Da}$

Peptide sequence: KKKKKQBKKKHRWPKXXA
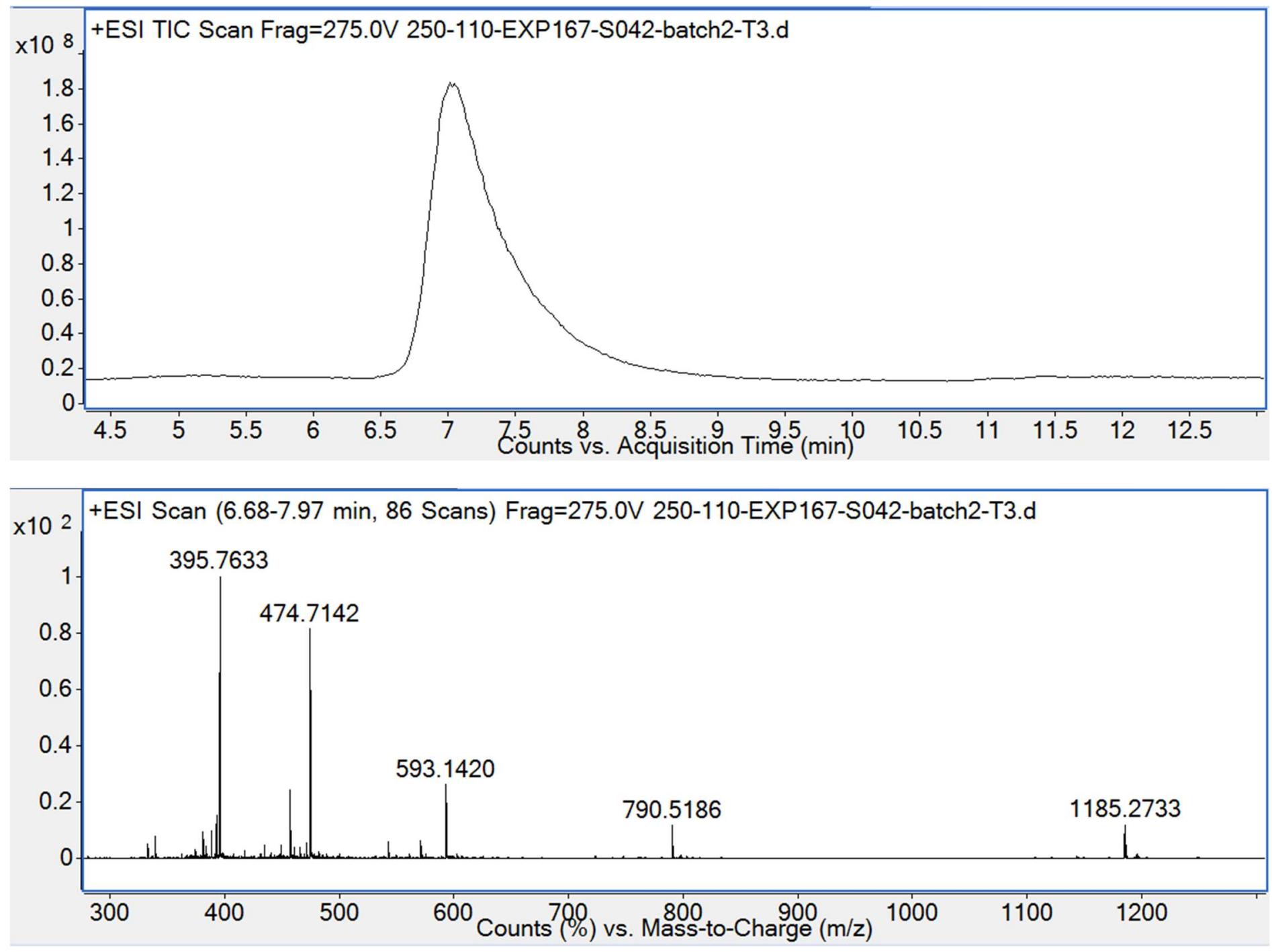
azide-P9

Mass expected: $2357.5 \mathrm{Da}$

Mass observed: 2358.5 Da

Peptide sequence: KKKKKQBKKKHRWPKXAC
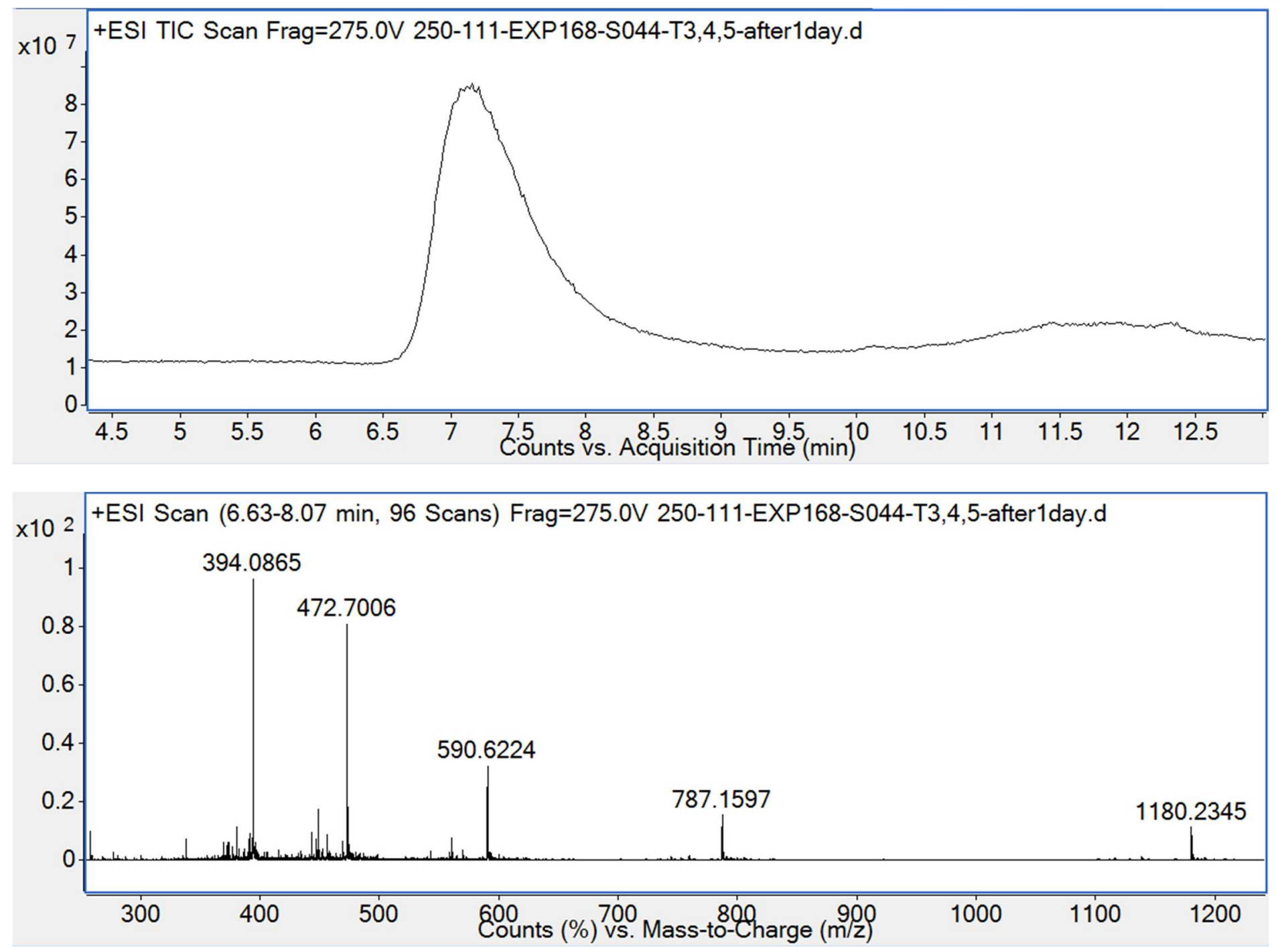


\section{azide-P10}

Mass expected: $2357.5 \mathrm{Da}$

Mass observed: $2358.5 \mathrm{Da}$

Peptide sequence: KKKKKQBKKKHRWPKAXC
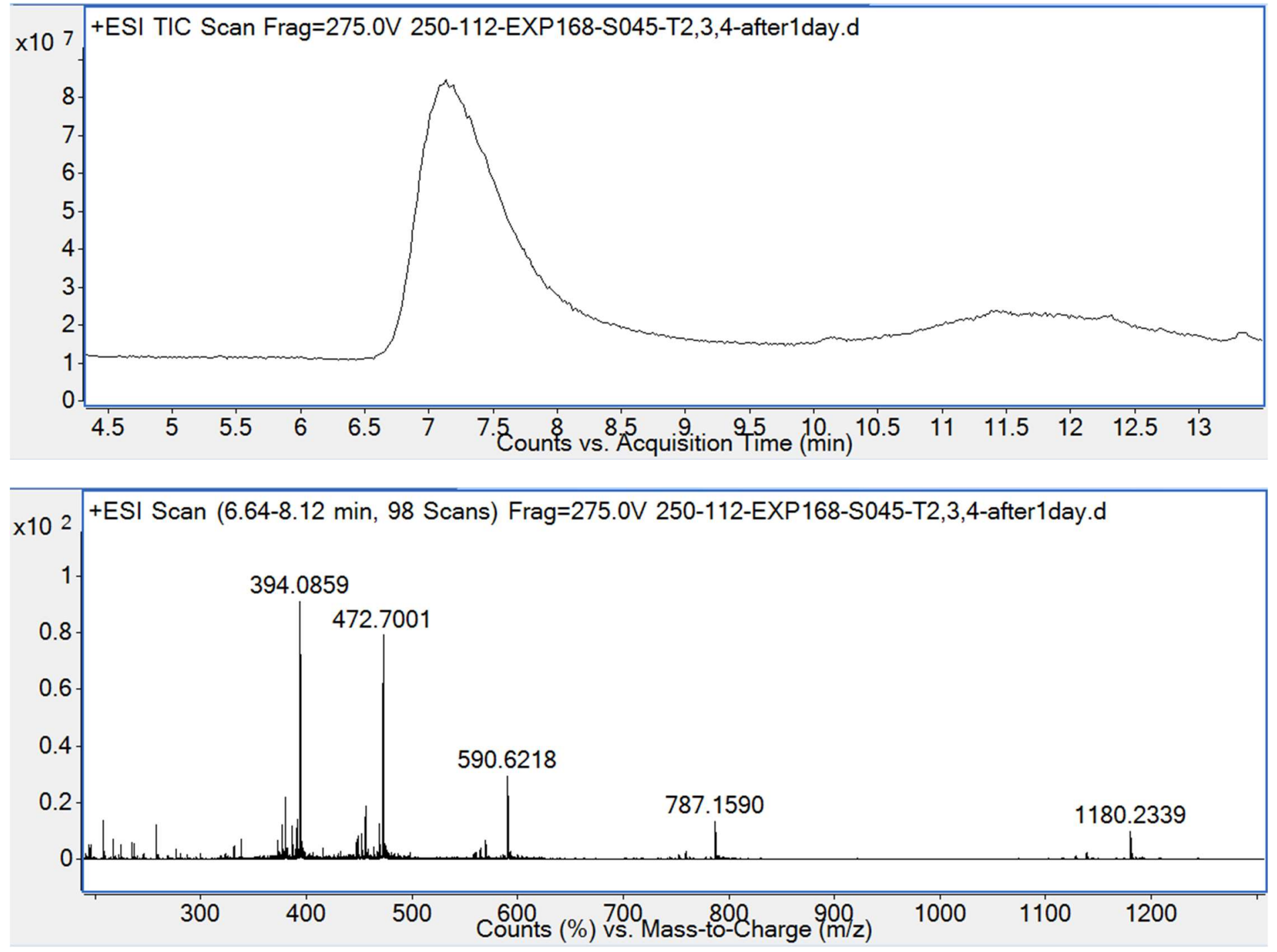
azide-P11

Mass expected: $2342.5 \mathrm{Da}$

Mass observed: 2343.5 Da

Peptide sequence: KKKKKQBKKKHRWPAXXC
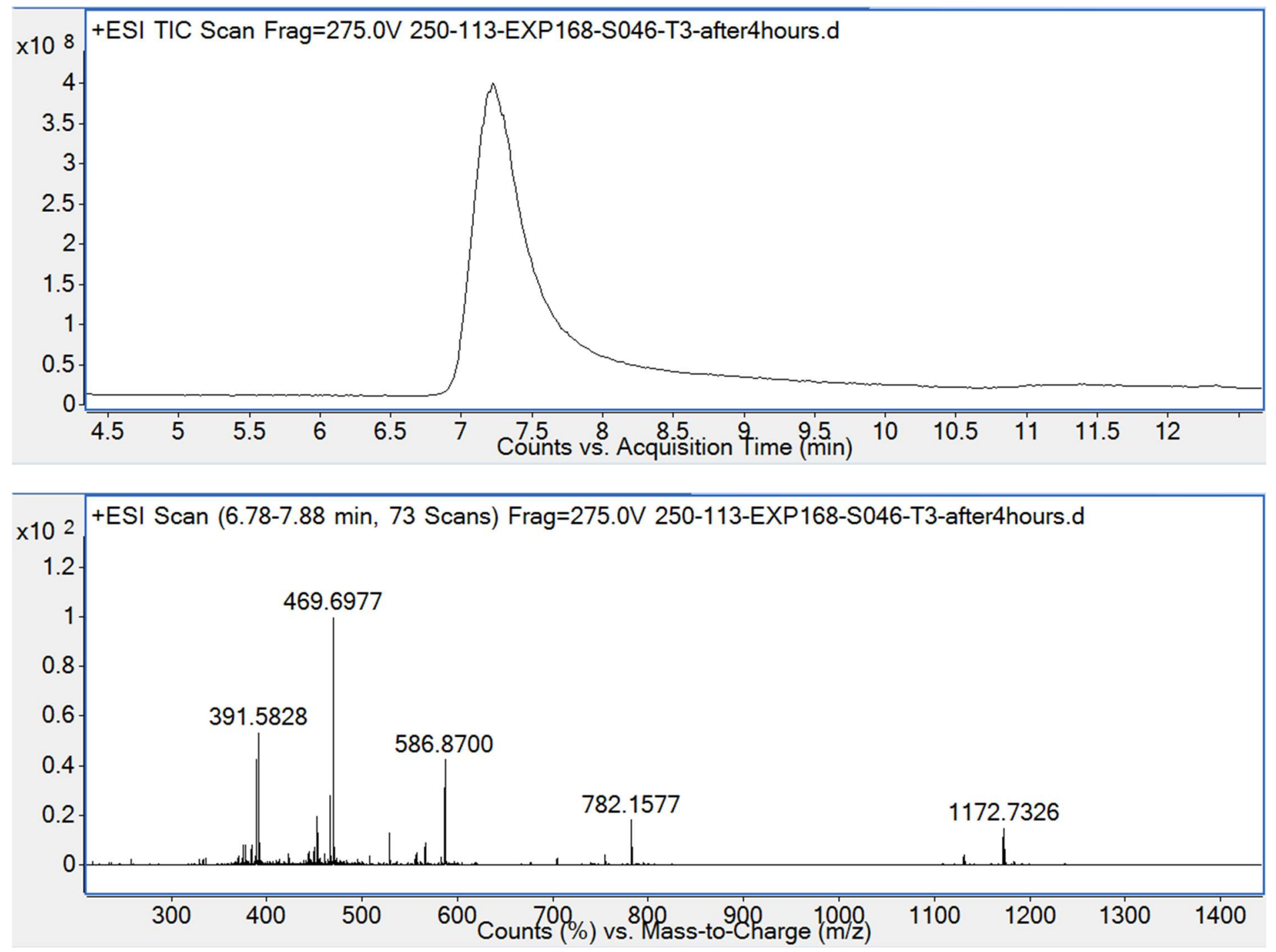


\section{azide-P12}

Mass expected: 1943.2 Da

Mass observed: 1943.3 Da

Peptide sequence: KKKKKQBKKKHRWP
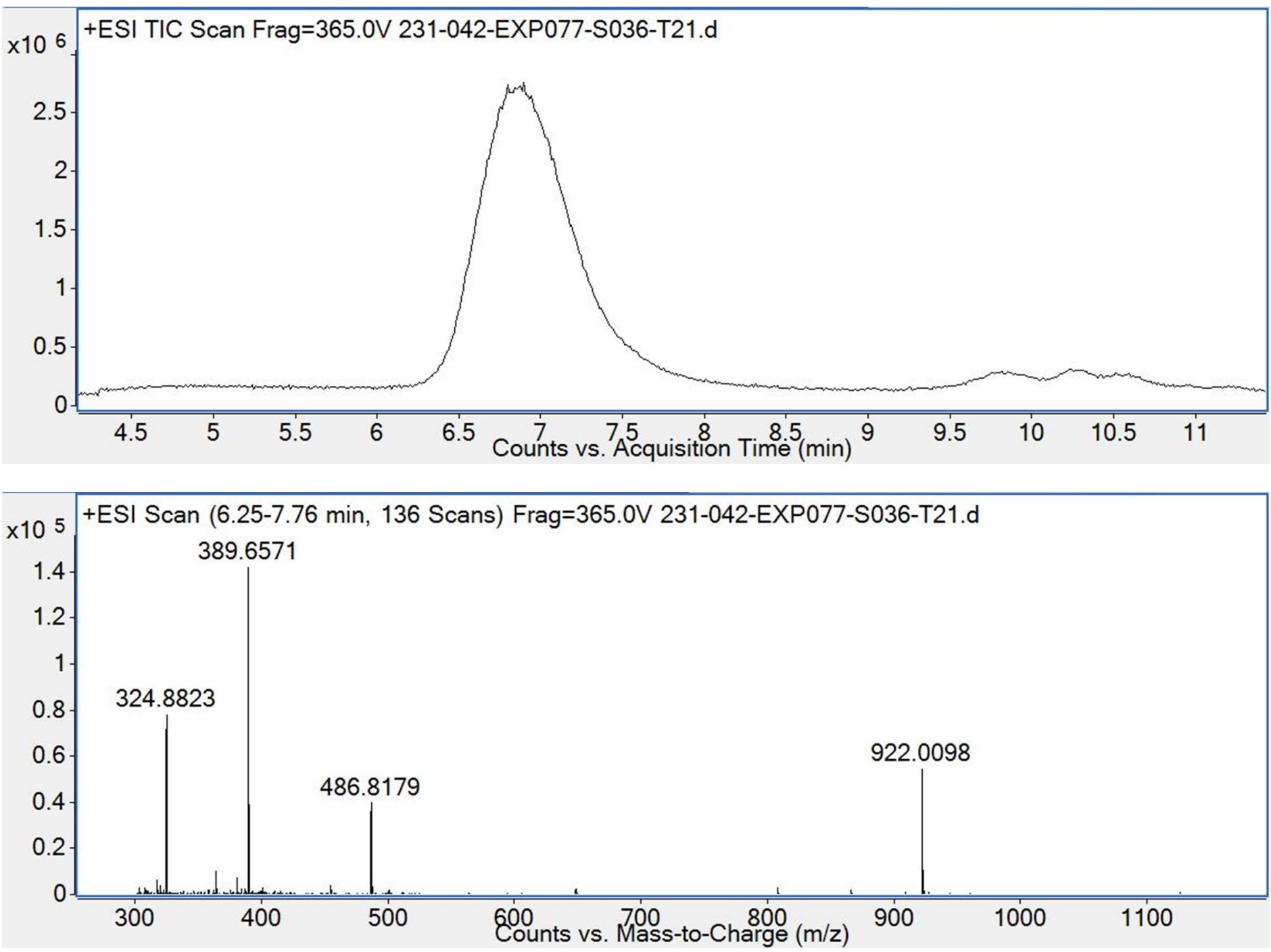
azide-P13

Mass expected: $2342.5 \mathrm{Da}$

Mass observed: $2343.5 \mathrm{Da}$

Peptide sequence: KAKKKQBKKKHRWPKXXC
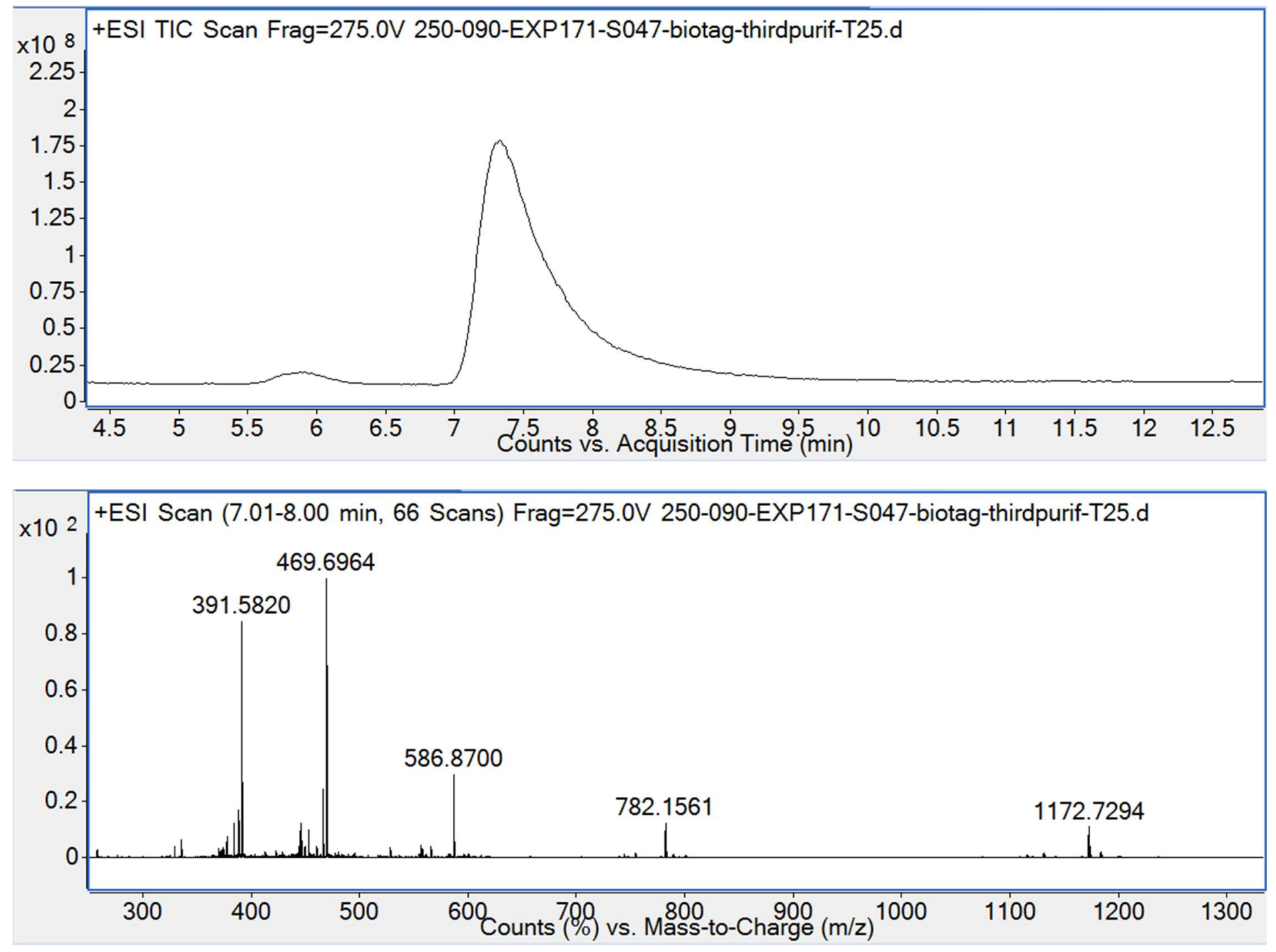


\section{azide-P14}

Mass expected: $2342.5 \mathrm{Da}$

Mass observed: 2343.5 Da

Peptide sequence: KKKKKQBKAKHRWPKXXC
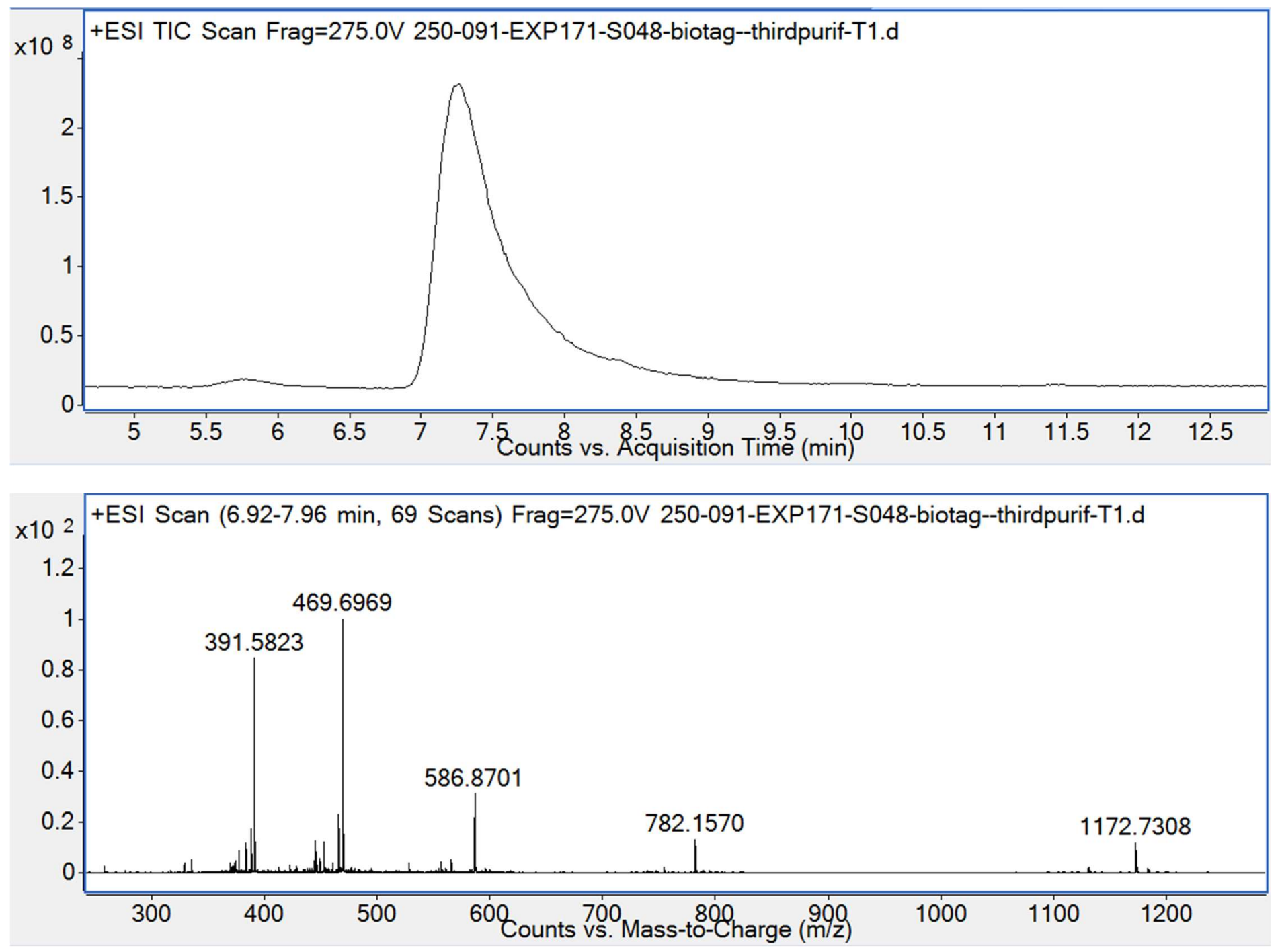


\section{azide-P15}

Mass expected: $2342.5 \mathrm{Da}$

Mass observed: 2343.5 Da

Peptide sequence: KKKAKQBKKKHRWPKXXC
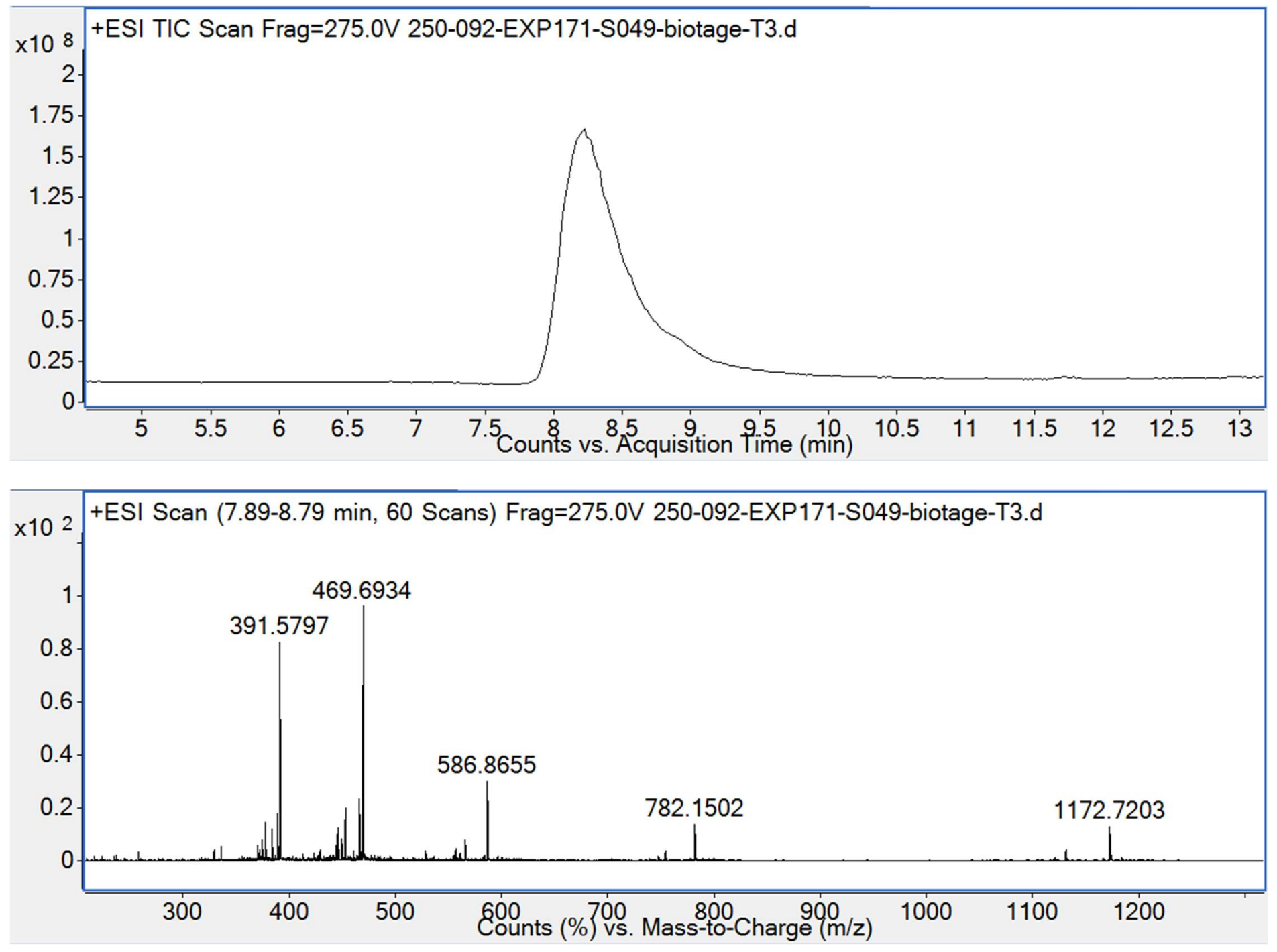


\section{azide-P16}

Mass expected: $2285.4 \mathrm{Da}$

Mass observed: 2286.4 Da

Peptide sequence: KAKKKQBKAKHRWPKXXC
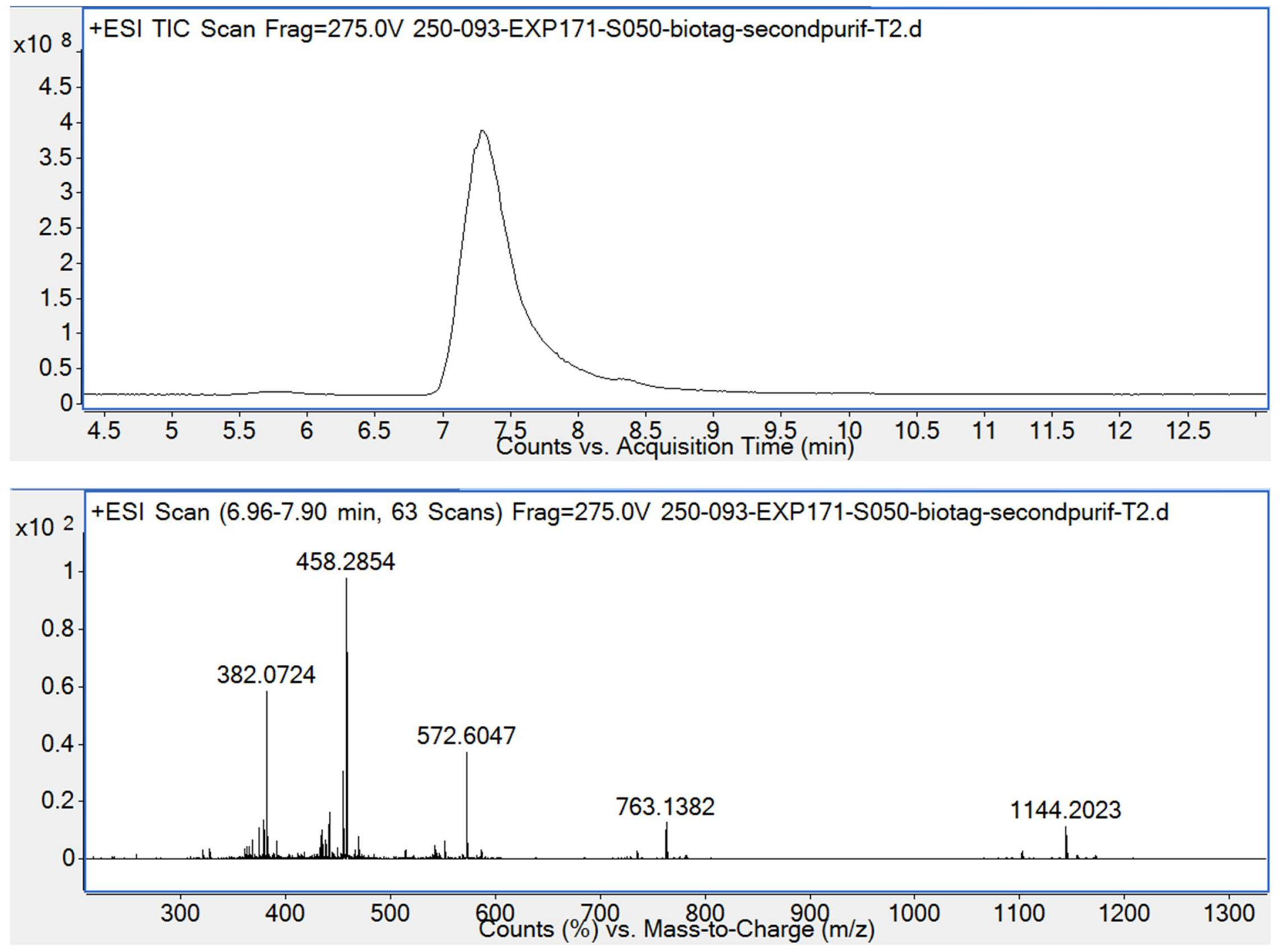
azide-P17

Mass expected: $2285.4 \mathrm{Da}$

Mass observed: 2286.4 Da

Peptide sequence: KAKAKQBKKKHRWPKXXC
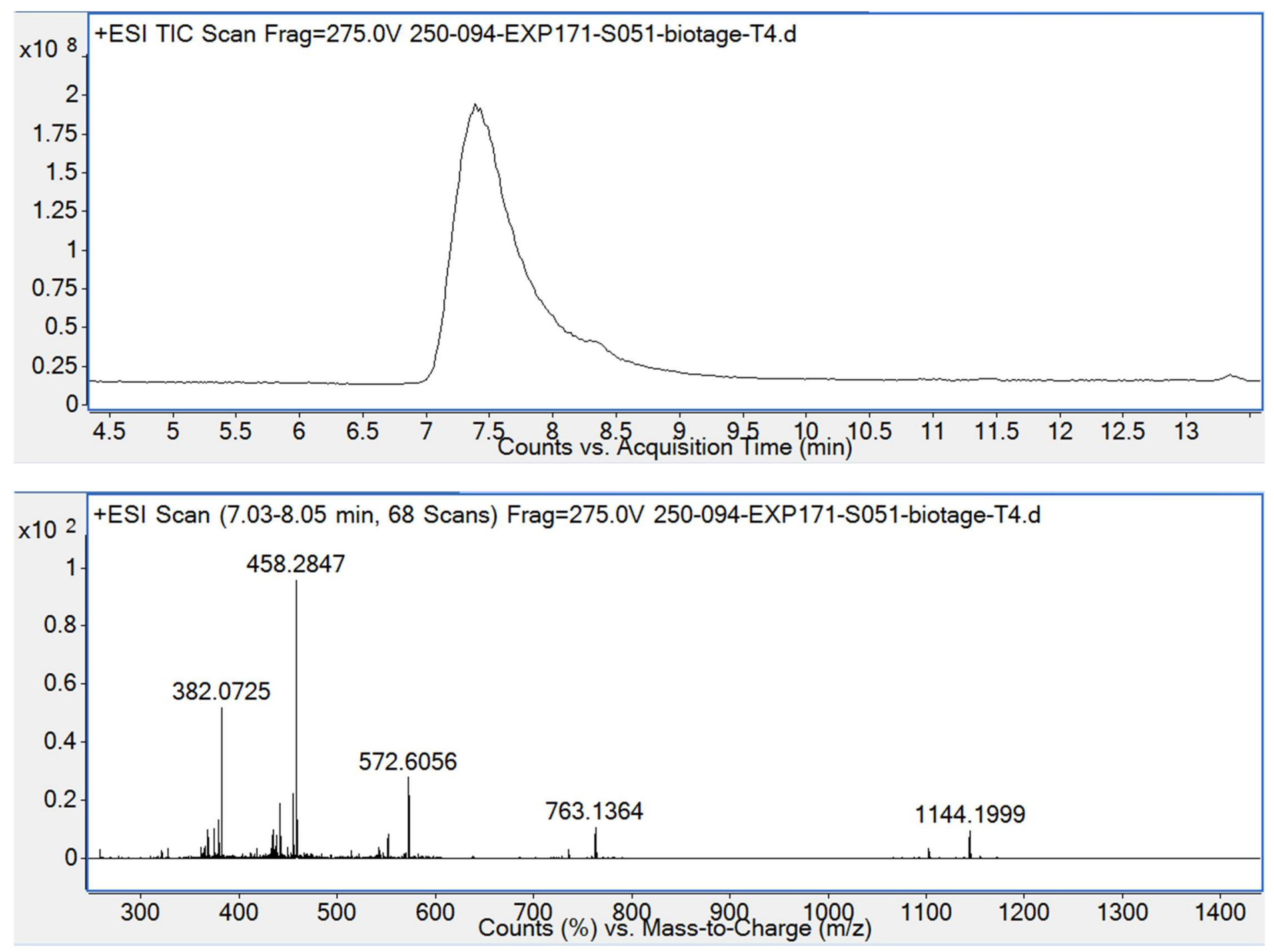


\section{azide-P18}

Mass expected: $2228.3 \mathrm{Da}$

Mass observed: 2229.4 Da

Peptide sequence: KAKAKQBKAKHRWPKXXC
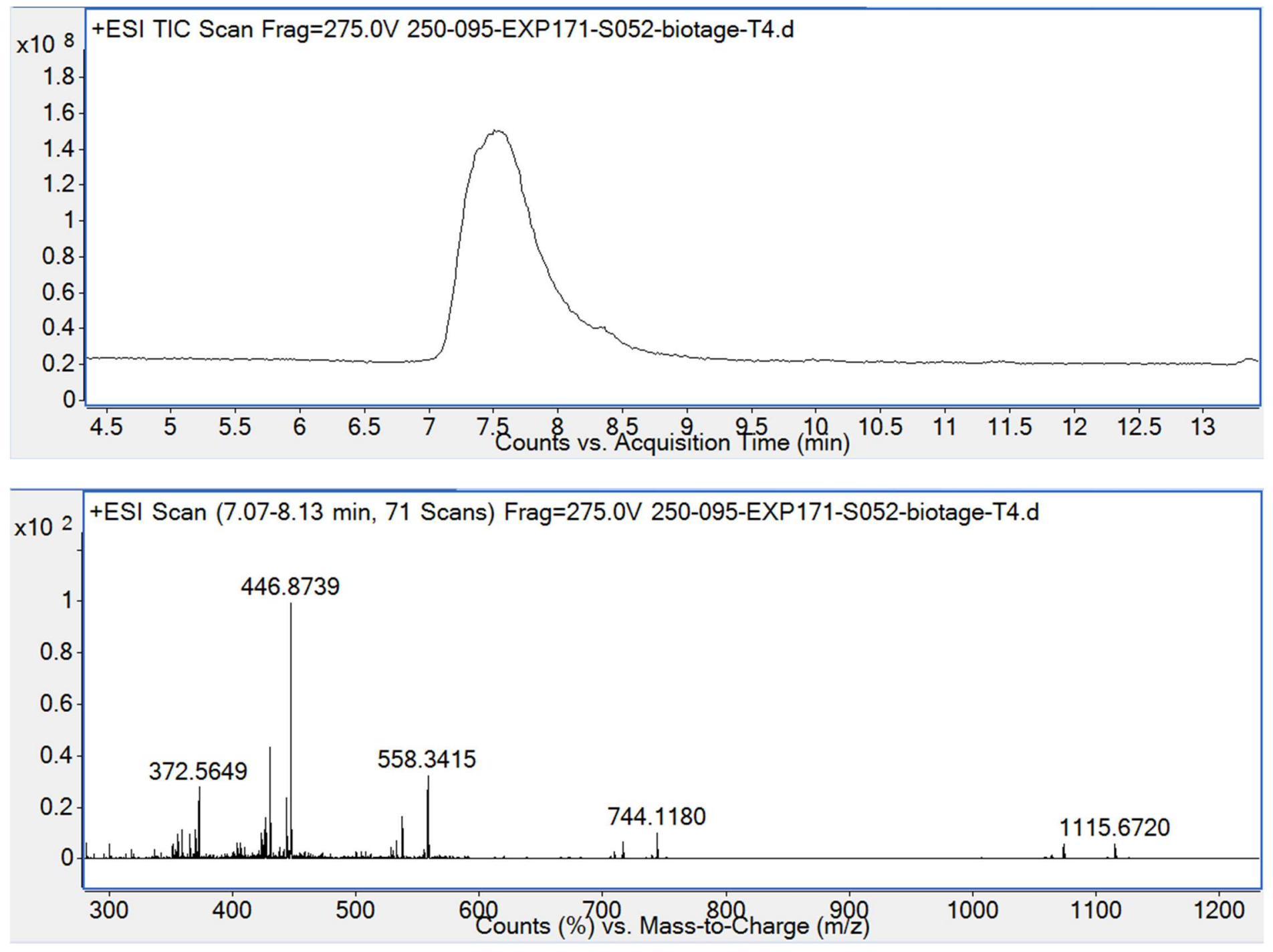


\section{azide-P19}

Mass expected: $2314.4 \mathrm{Da}$

Mass observed: $2314.5 \mathrm{Da}$

Peptide sequence: KKKKKQBKKKHAWPKXXC
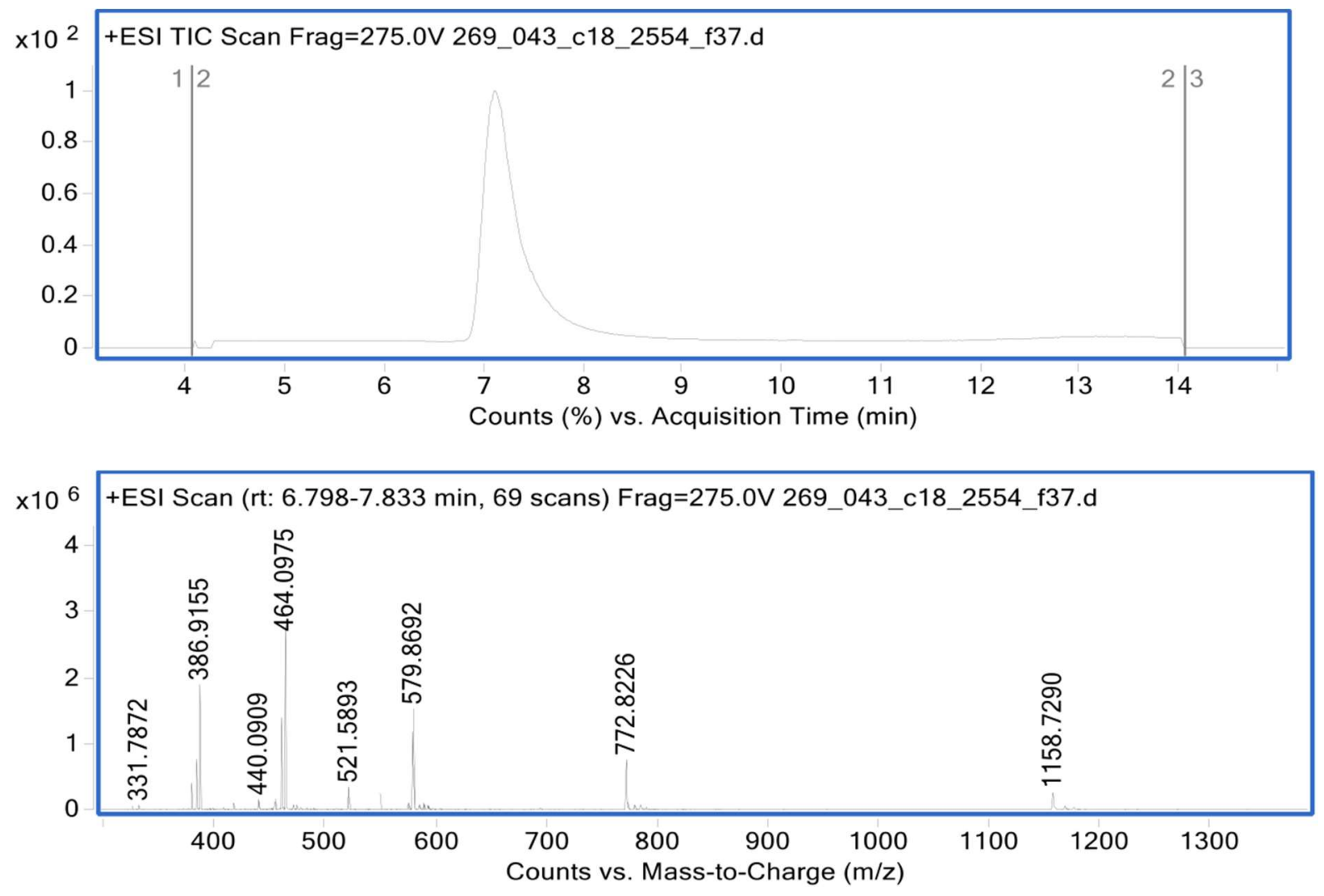


\section{P6-LPSTGG}

Mass expected: $2788.5 \mathrm{Da}$

Mass observed: $2787.8 \mathrm{Da}$

Peptide sequence: KKKKKQBKKKHRWPKXXCLPSTGG
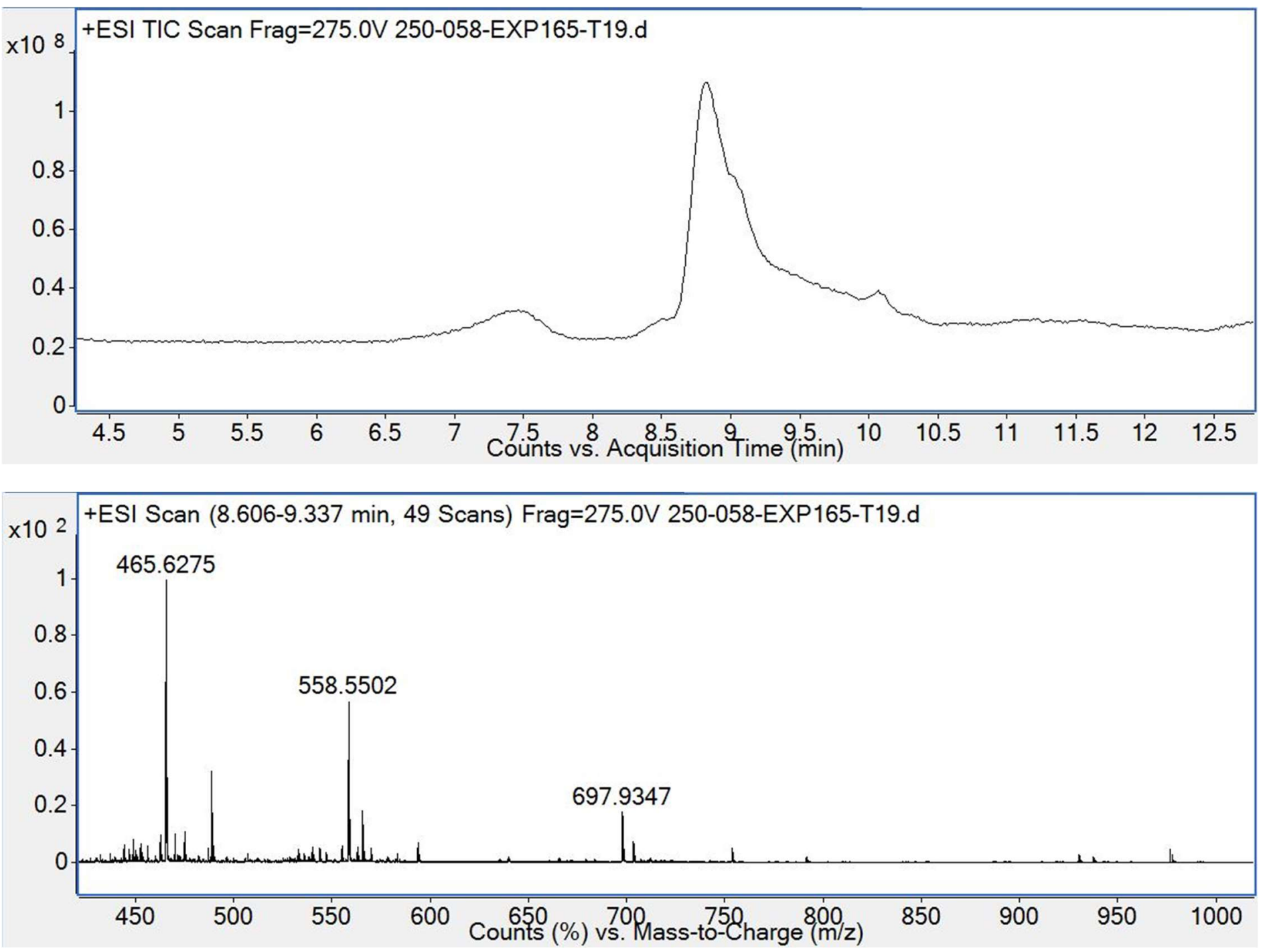


\section{PMO-P1}

Mass expected: $7253.9 \mathrm{Da}$

Mass observed: $7254.0 \mathrm{Da}$

Peptide sequence: KXKXT
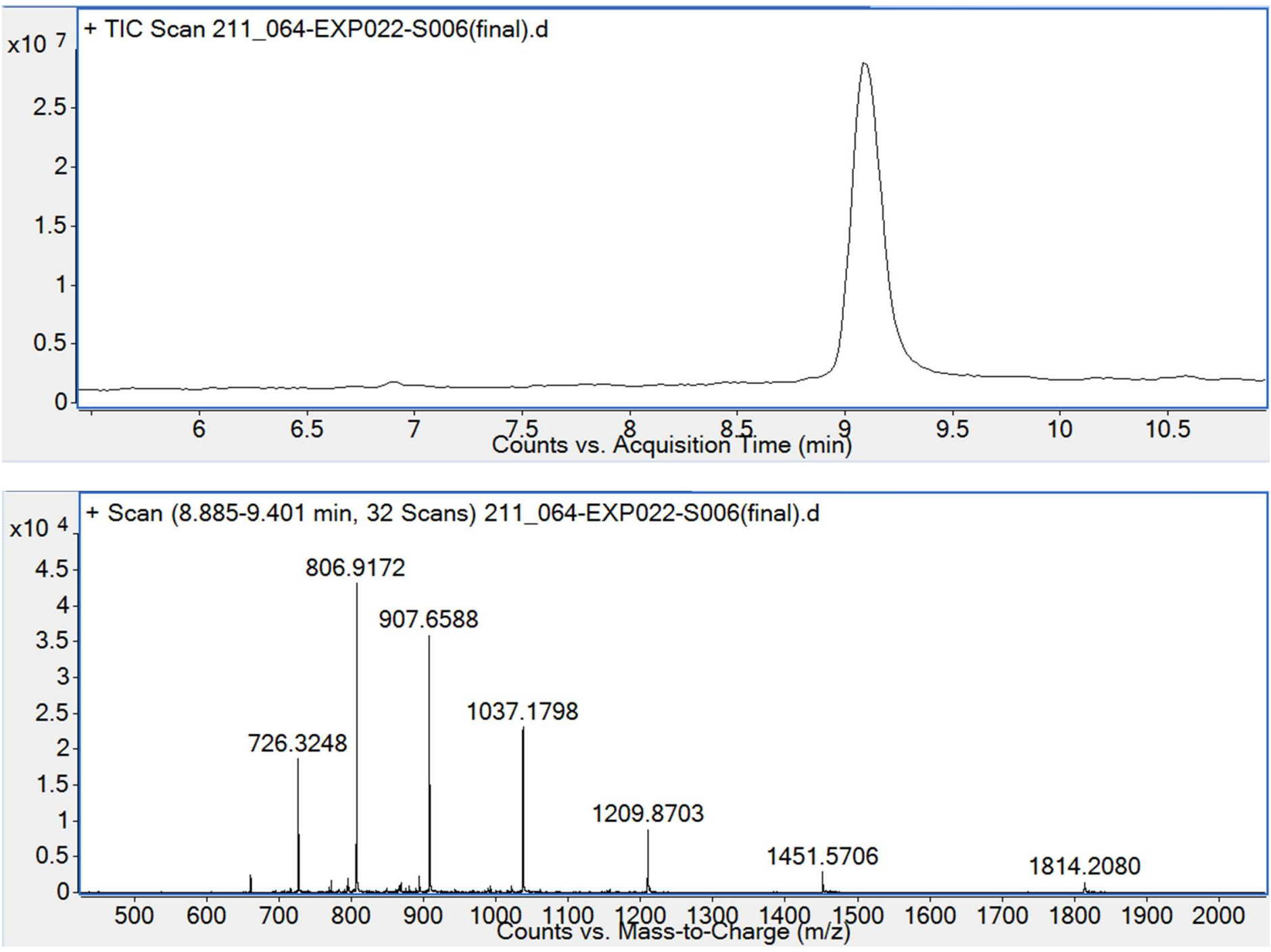


\section{PMO-P2}

Mass expected: $7802.5 \mathrm{Da}$

Mass observed: 7802.7 Da

Peptide sequence: KXKHQQQXK
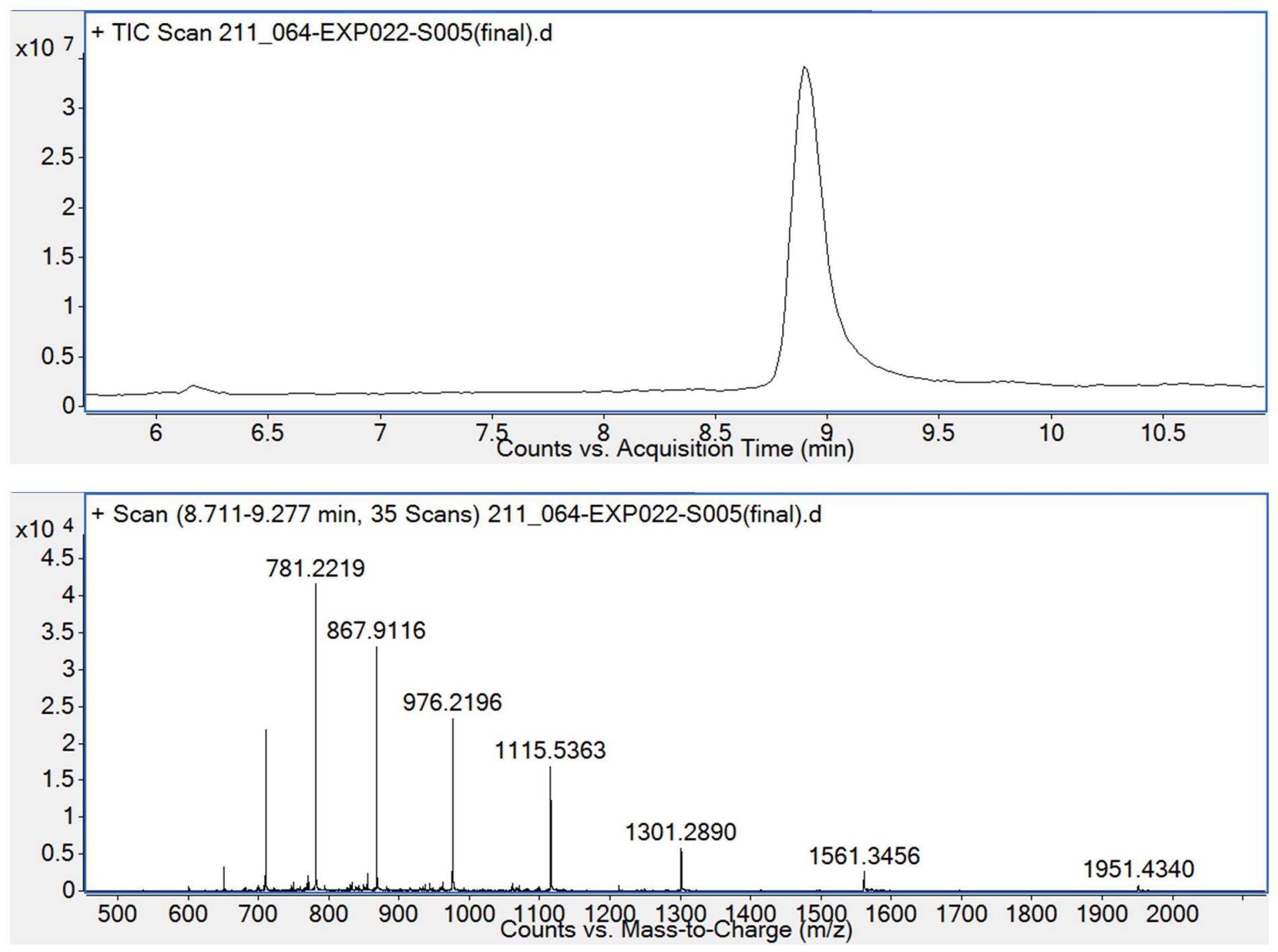


\section{PMO-P3}

Mass expected: $8159.0 \mathrm{Da}$

Mass observed: 8159.1 Da

Peptide sequence: KXKKQQGKKKHR
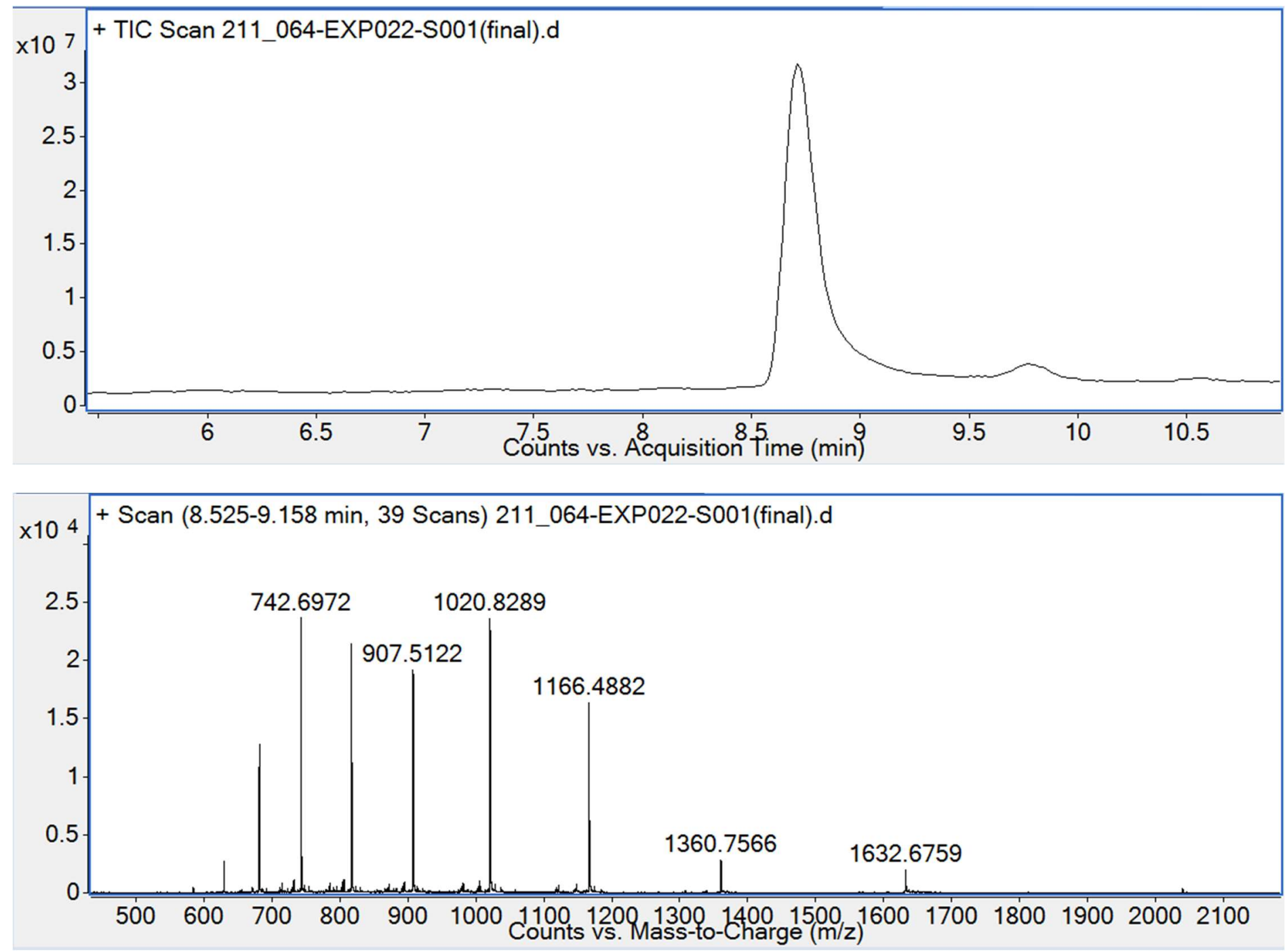


\section{PMO-P4}

Mass expected: $8480.4 \mathrm{Da}$

Mass observed: $8480.4 \mathrm{Da}$

Peptide sequence: HKKKKQBKKKHRWP
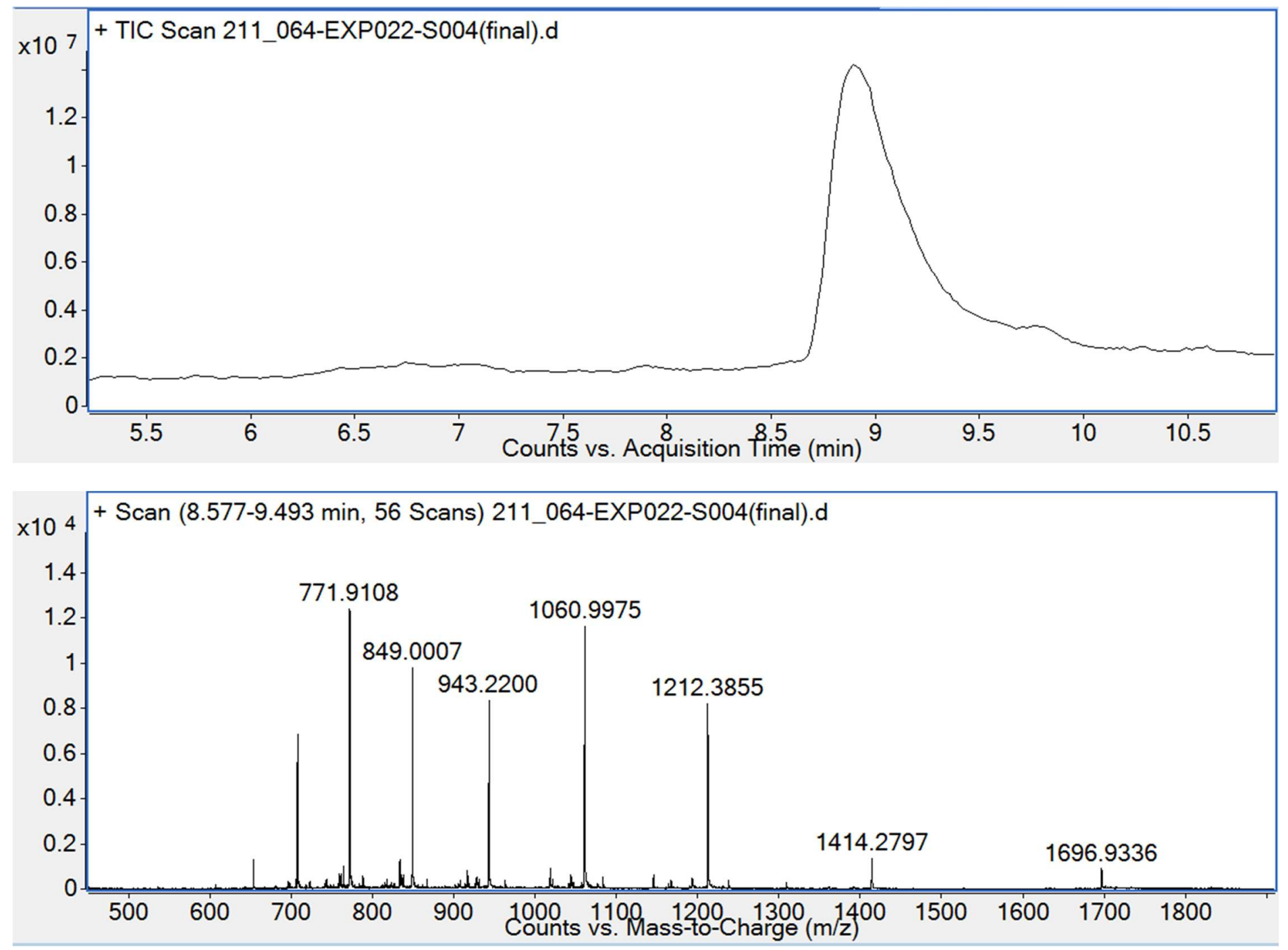


\section{PMO-P5}

Mass expected: $8659.7 \mathrm{Da}$

Mass observed: 8659.6 Da

Peptide sequence: KKKKKQBKKKHRWPMG
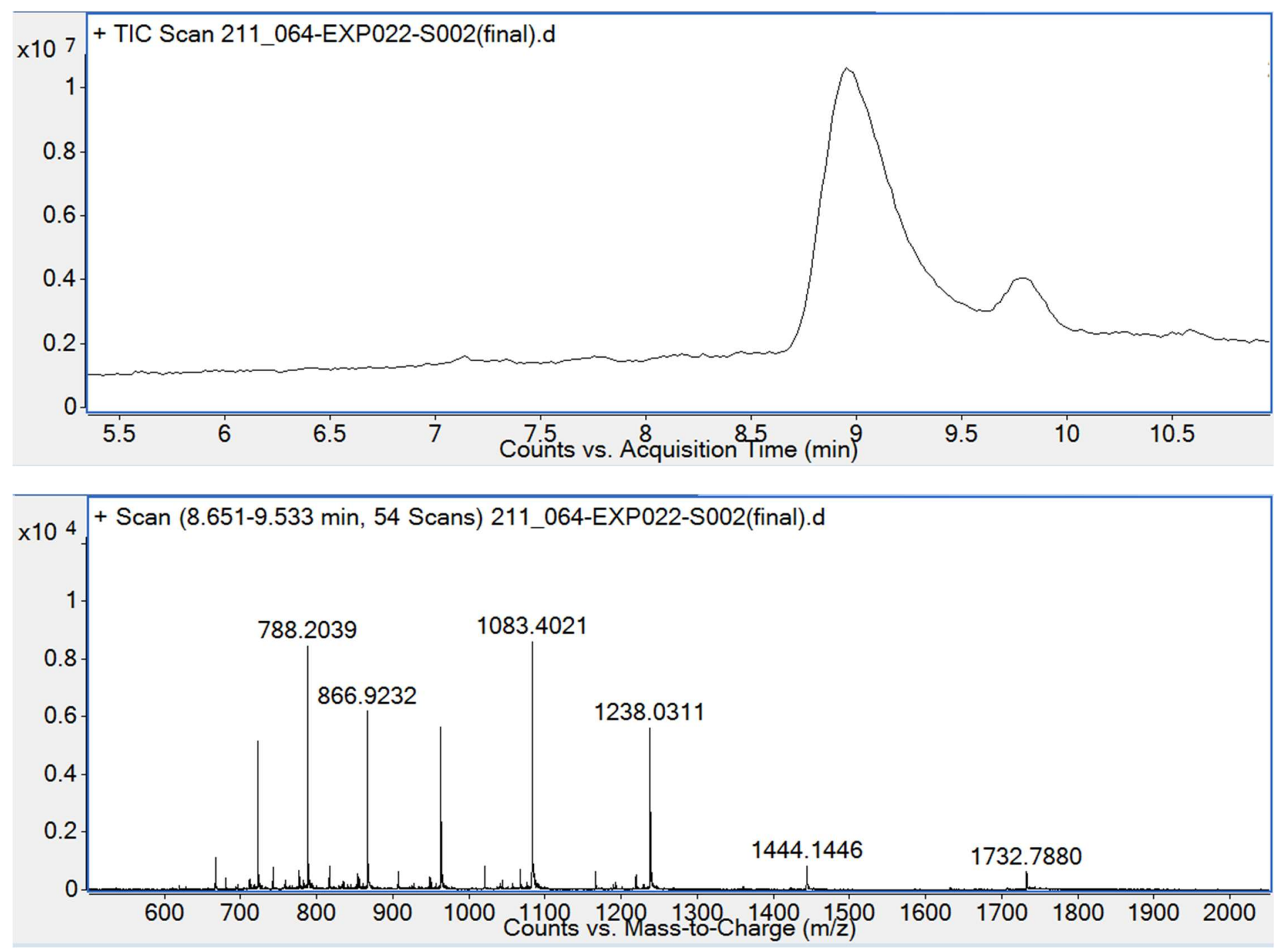


\section{PMO-P6}

Mass expected: $8929.1 \mathrm{Da}$

Mass observed: $8929.1 \mathrm{Da}$

Peptide sequence: KKKKKQBKKKHRWPKXXC
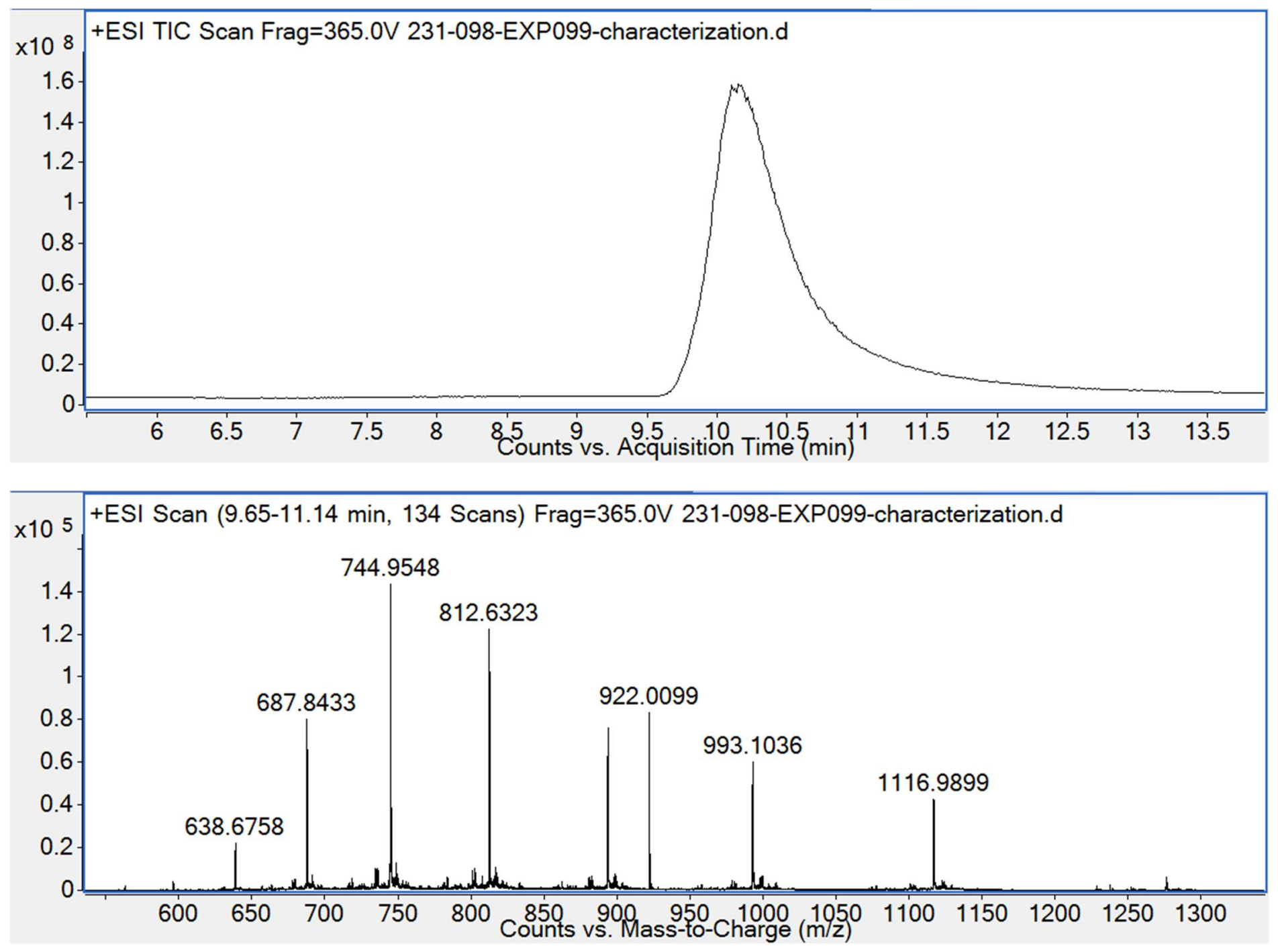


\section{PMO-P7}

Mass expected: $9158.3 \mathrm{Da}$

Mass observed: 9158.3 Da

Peptide sequence: KKKKNQBKKKHRWPMKXCPQ
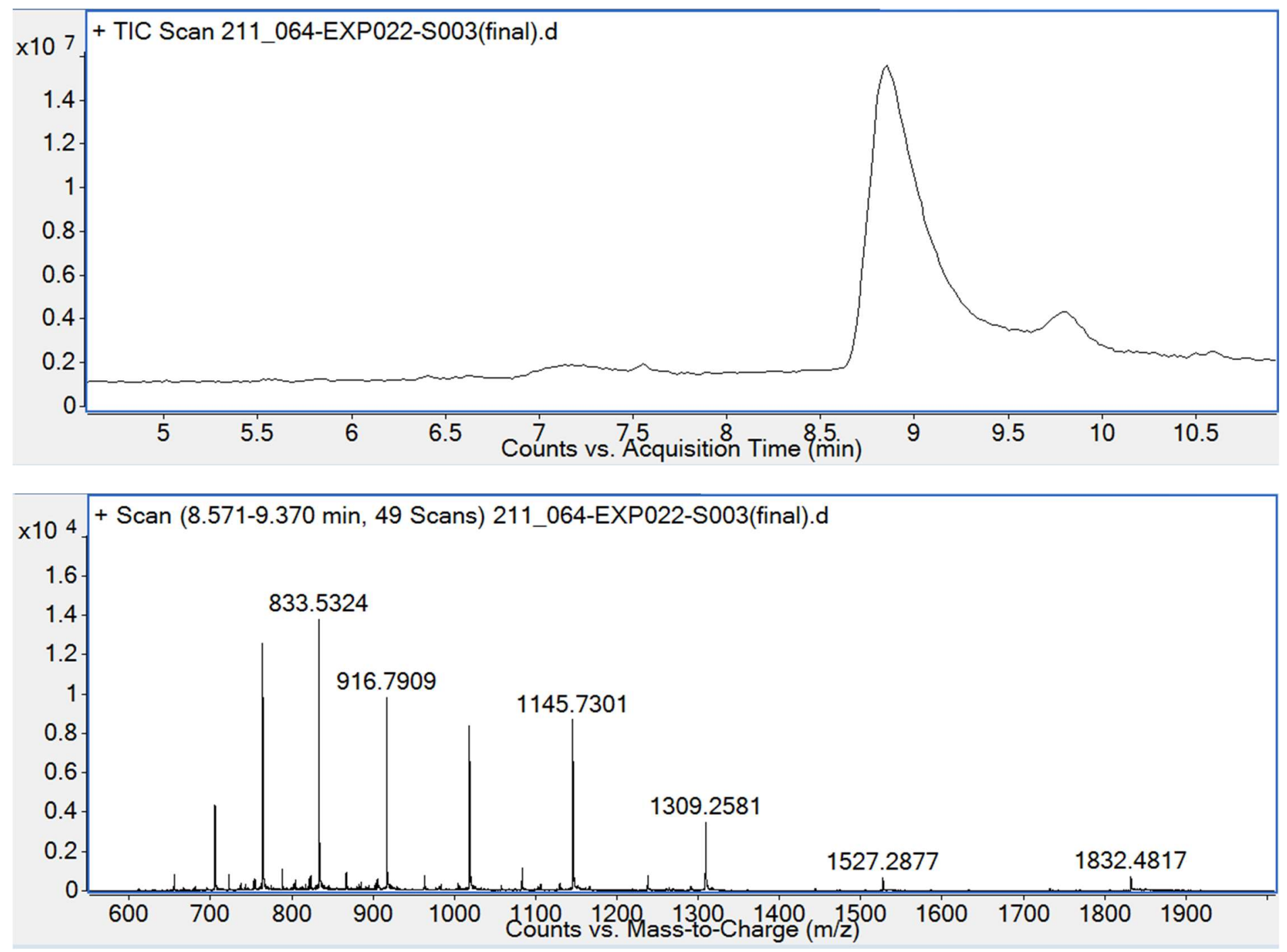


\section{PMO-P8}

Mass expected: $8895.5 \mathrm{Da}$

Mass observed: 8897.1 Da

Peptide sequence: KKKKKQBKKKHRWPKXXA
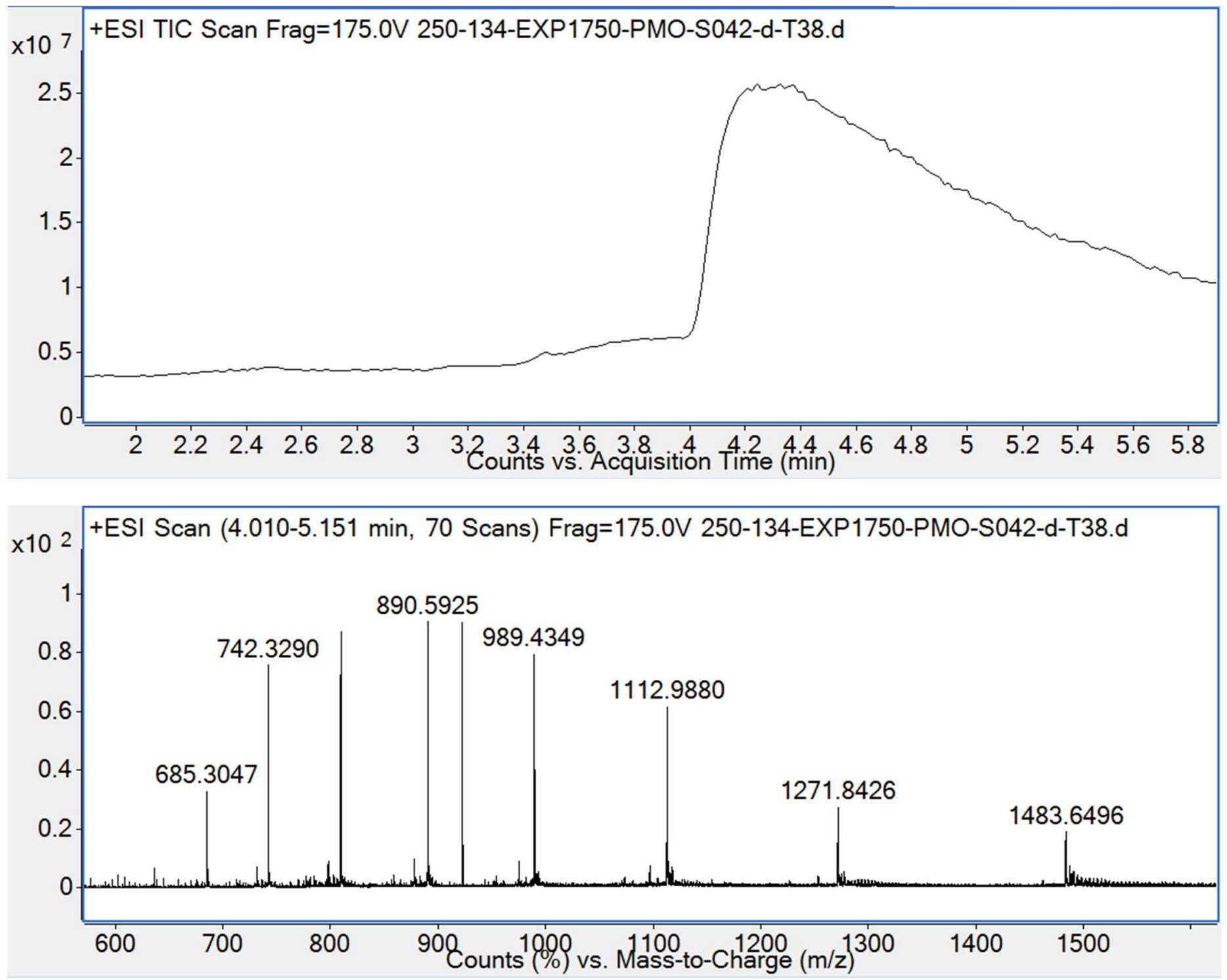


\section{PMO-P9}

Mass expected: $8885.5 \mathrm{Da}$

Mass observed: $8887.1 \mathrm{Da}$

Peptide sequence: KKKKKQBKKKHRWPKXAC
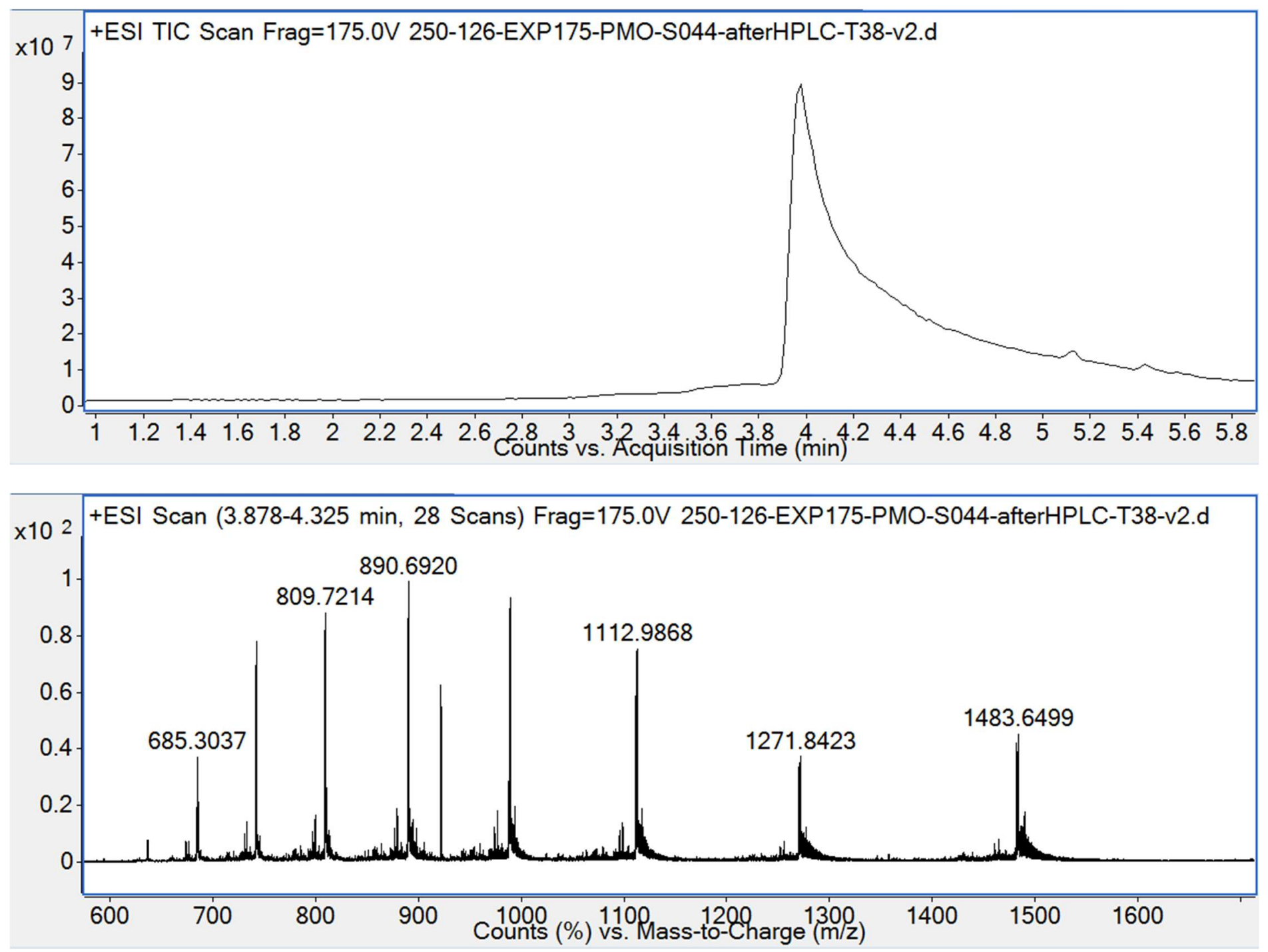


\section{PMO-P10}

Mass expected: $8885.5 \mathrm{Da}$

Mass observed: $8887.1 \mathrm{Da}$

Peptide sequence: KKKKKQBKKKHRWPKAXC
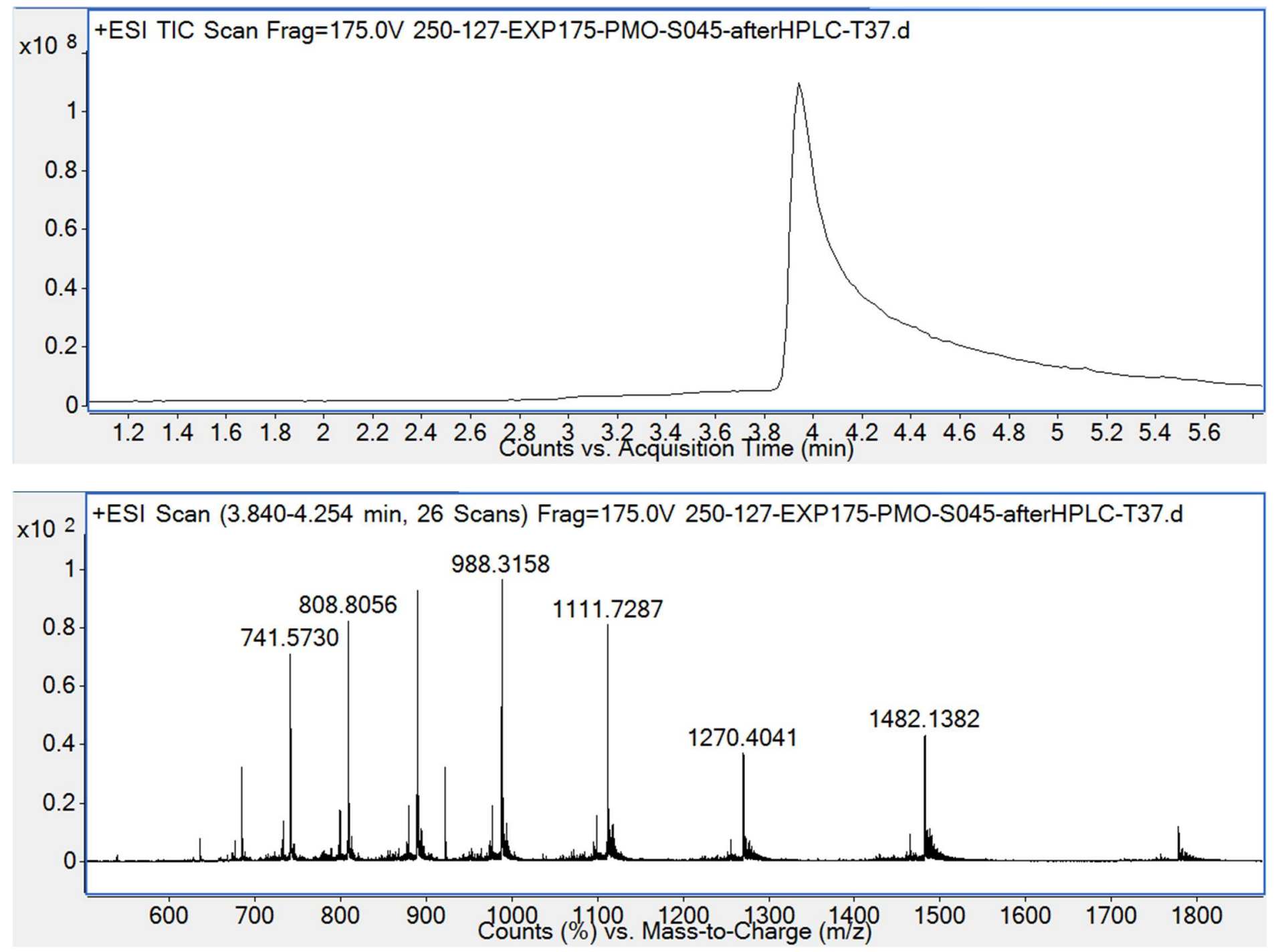


\section{PMO-P11}

Mass expected: $8870.5 \mathrm{Da}$

Mass observed: $8872.2 \mathrm{Da}$

Peptide sequence: KKKKKQBKKKHRWPAXXC

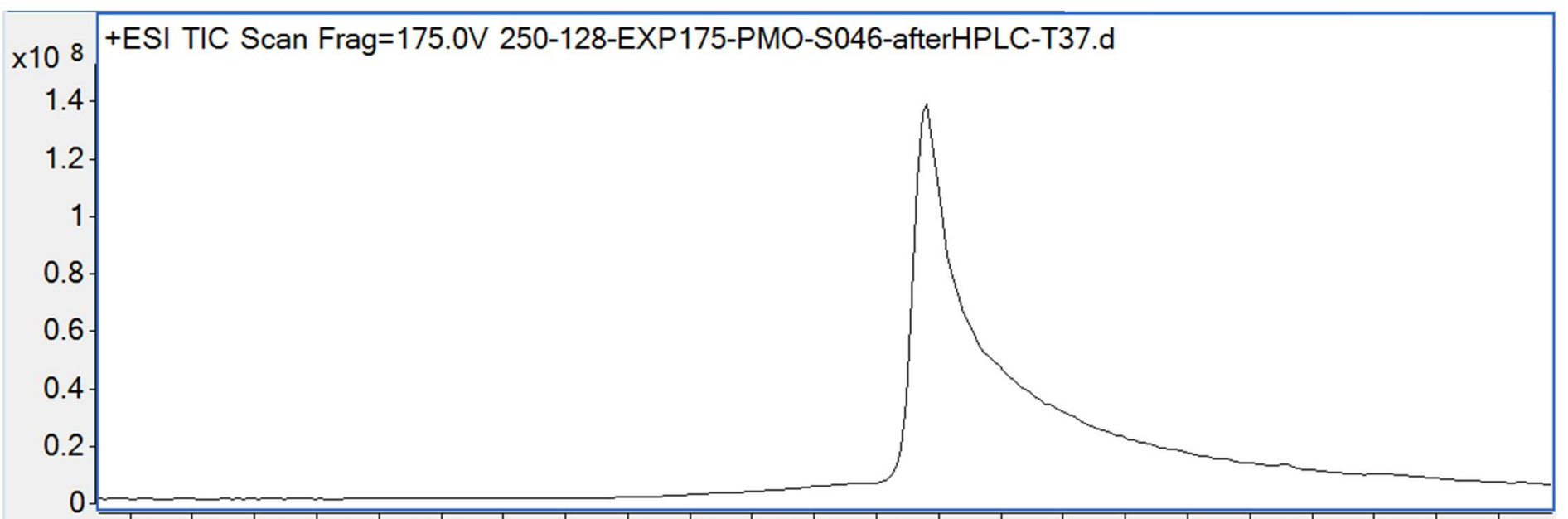

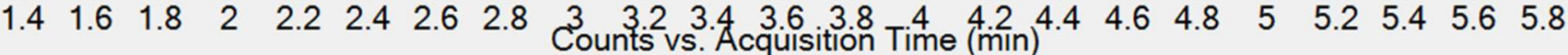

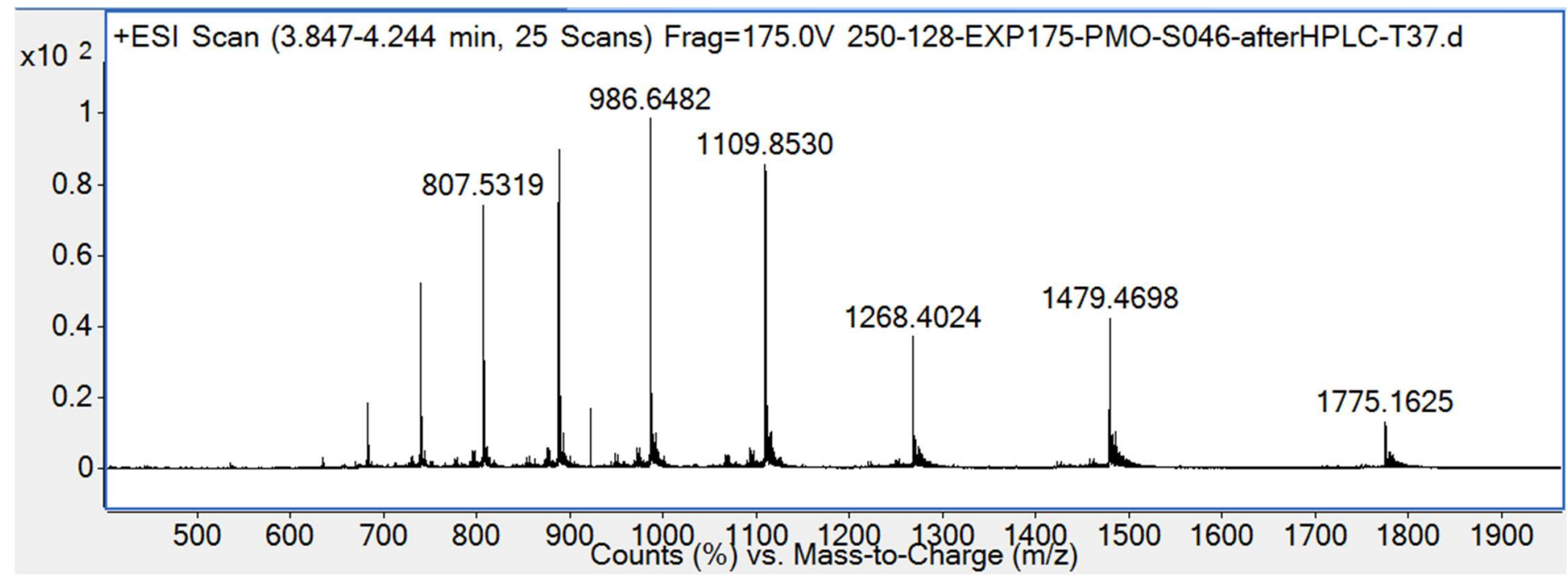




\section{PMO-P12}

Mass expected: $8471.4 \mathrm{Da}$

Mass observed: $8472.1 \mathrm{Da}$

Peptide sequence: KKKKKQBKKKHRWP
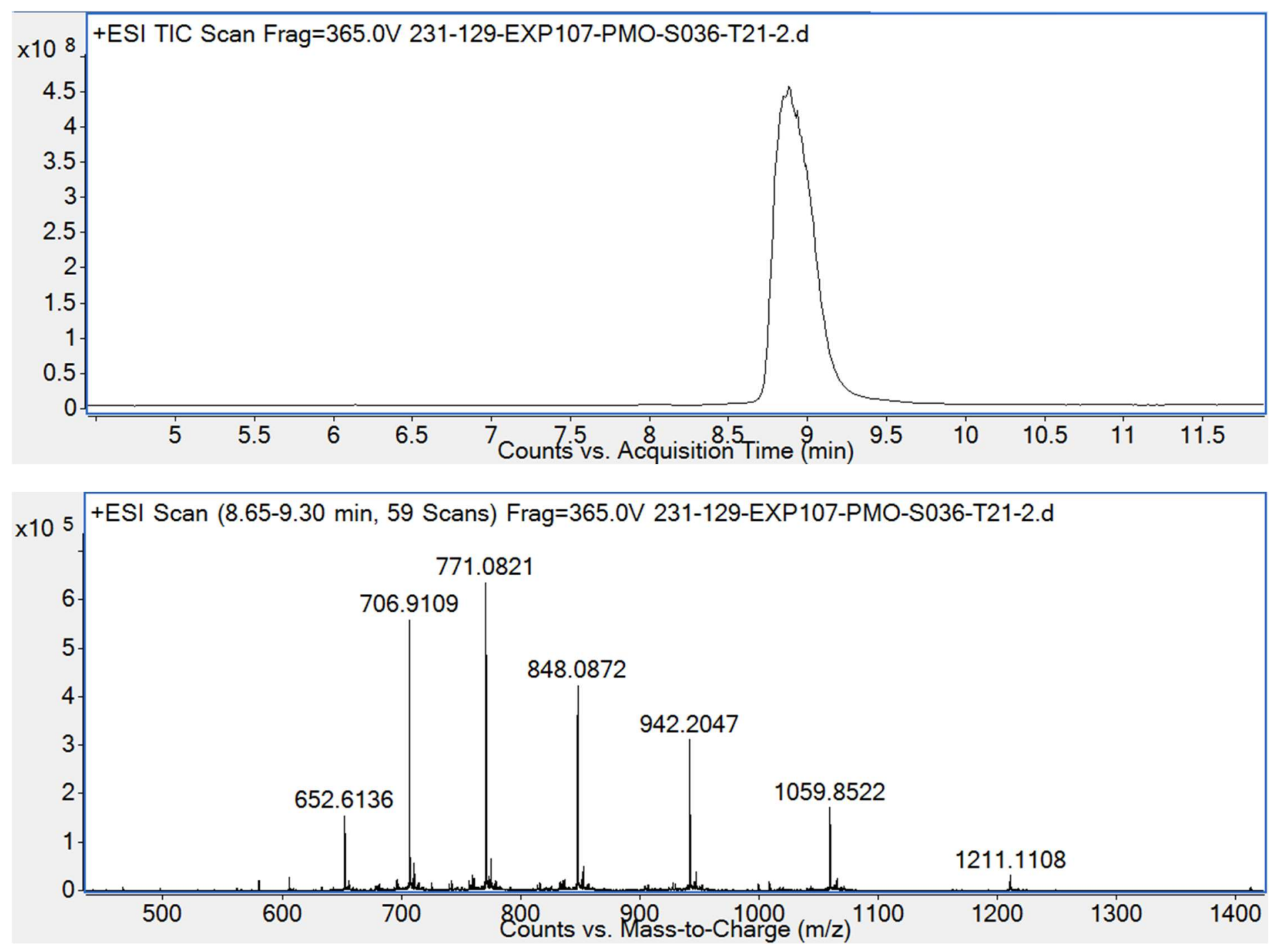


\section{PMO-P13}

Mass expected: $8871.5 \mathrm{Da}$

Mass observed: $8872.2 \mathrm{Da}$

Peptide sequence: KAKKKQBKKKHRWPKXXC
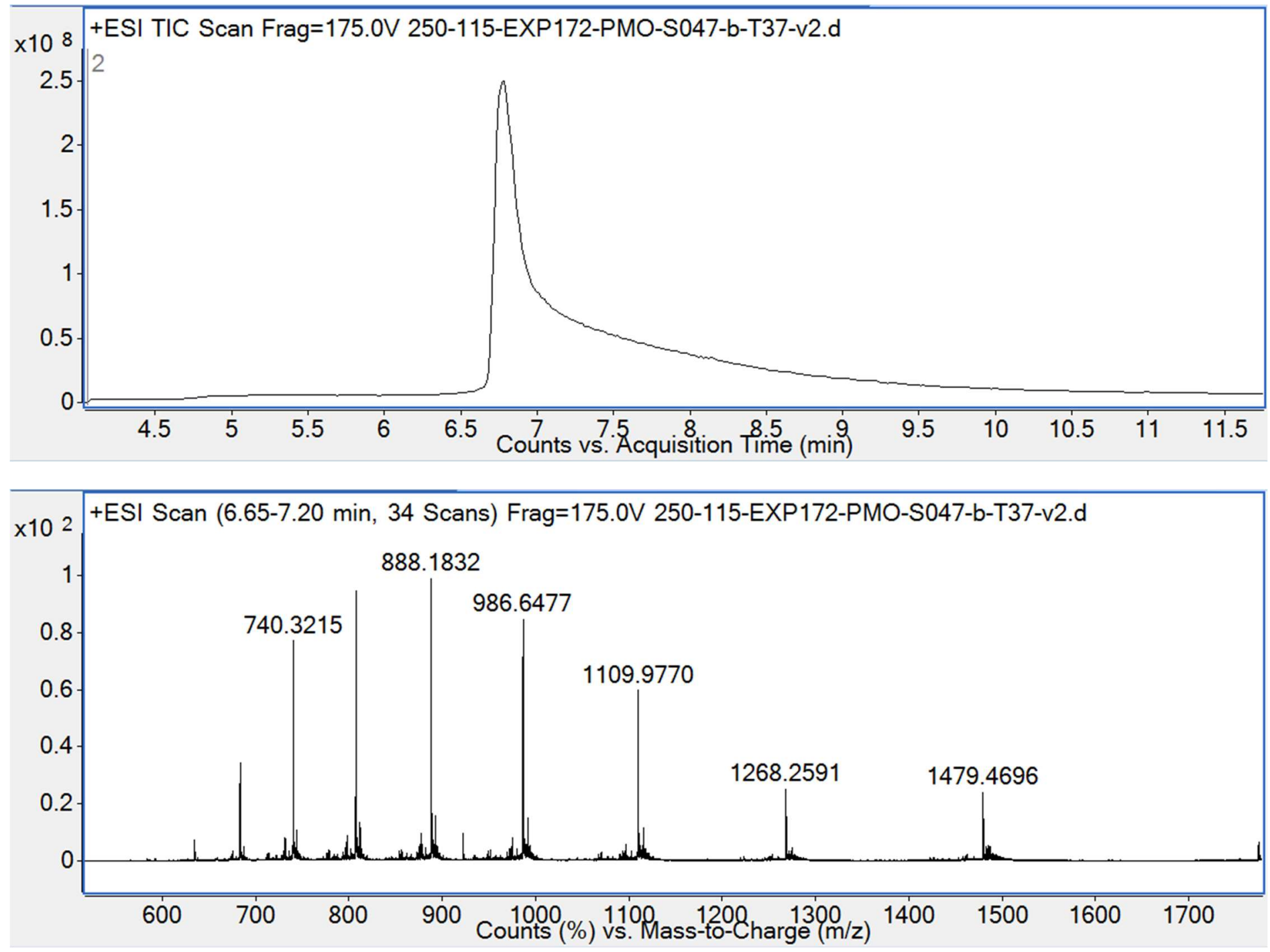


\section{PMO-P14}

Mass expected: $8871.5 \mathrm{Da}$

Mass observed: $8872.2 \mathrm{Da}$

Peptide sequence: KKKKKQBKAKHRWPKXXC
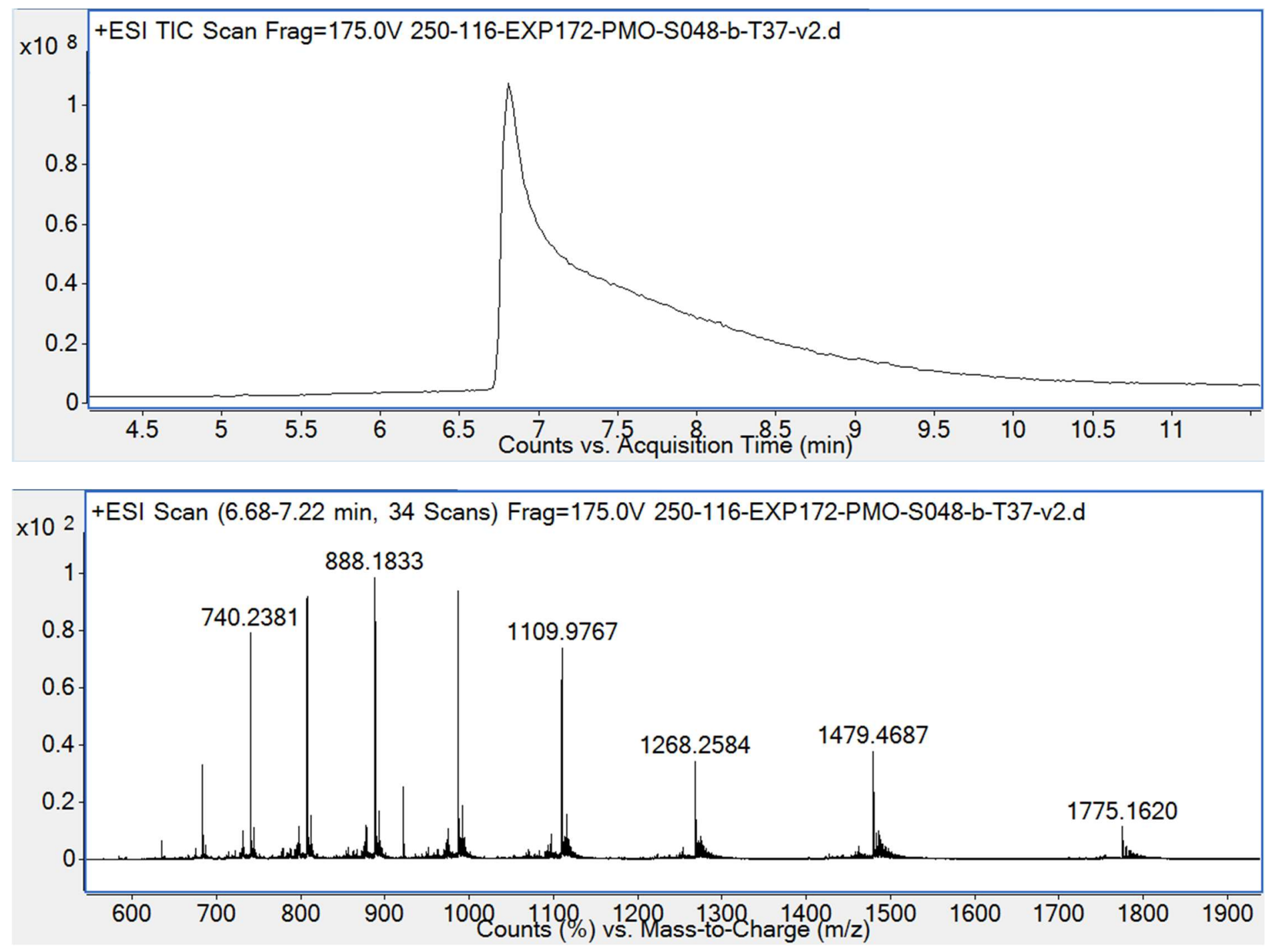


\section{PMO-P15}

Mass expected: $8871.5 \mathrm{Da}$

Mass observed: $8872.2 \mathrm{Da}$

Peptide sequence: KKKAKQBKKKHRWPKXXC
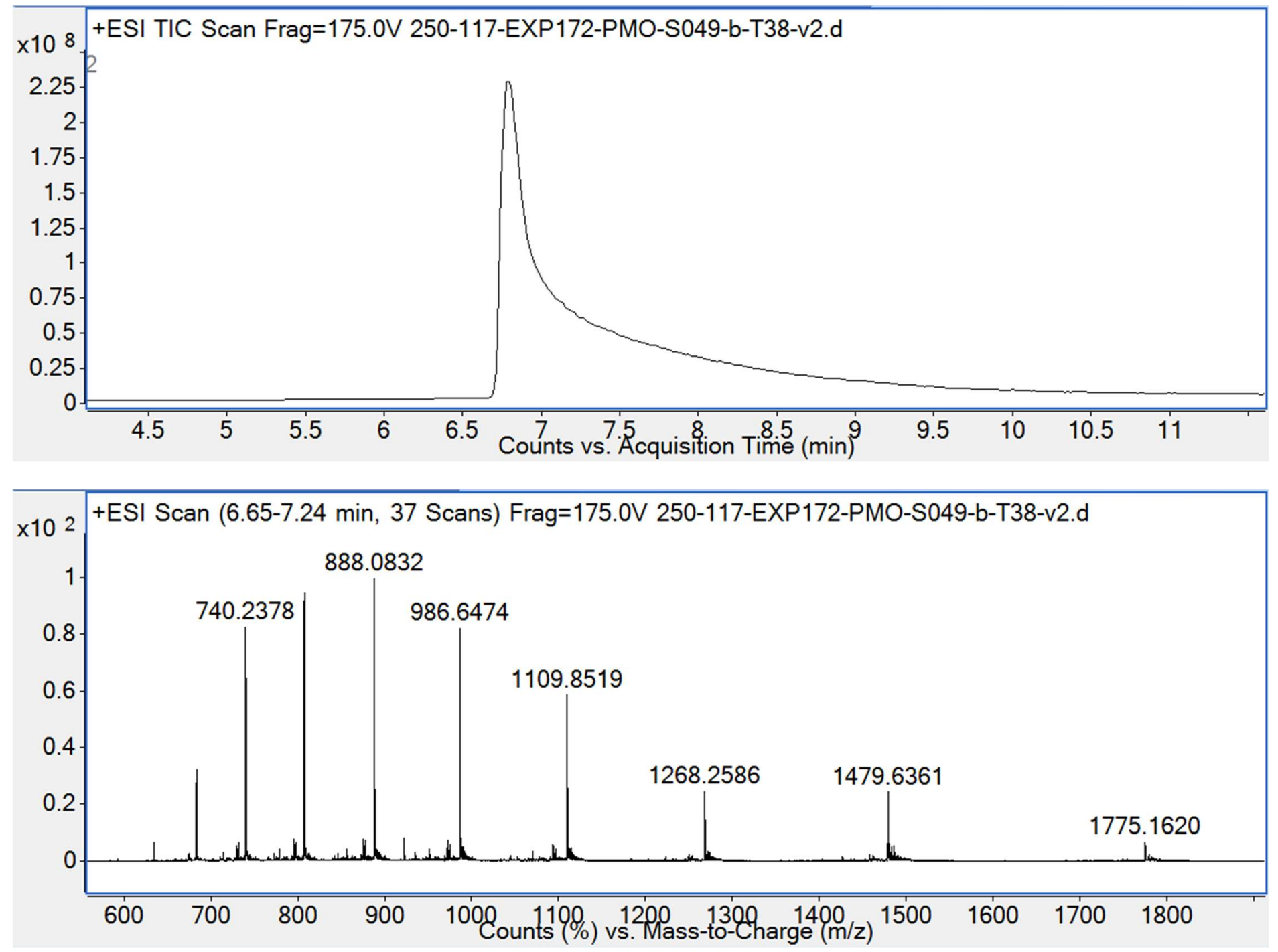


\section{PMO-P16}

Mass expected: $8814.4 \mathrm{Da}$

Mass observed: 8815.2 Da

Peptide sequence: KAKKKQBKAKHRWPKXXC
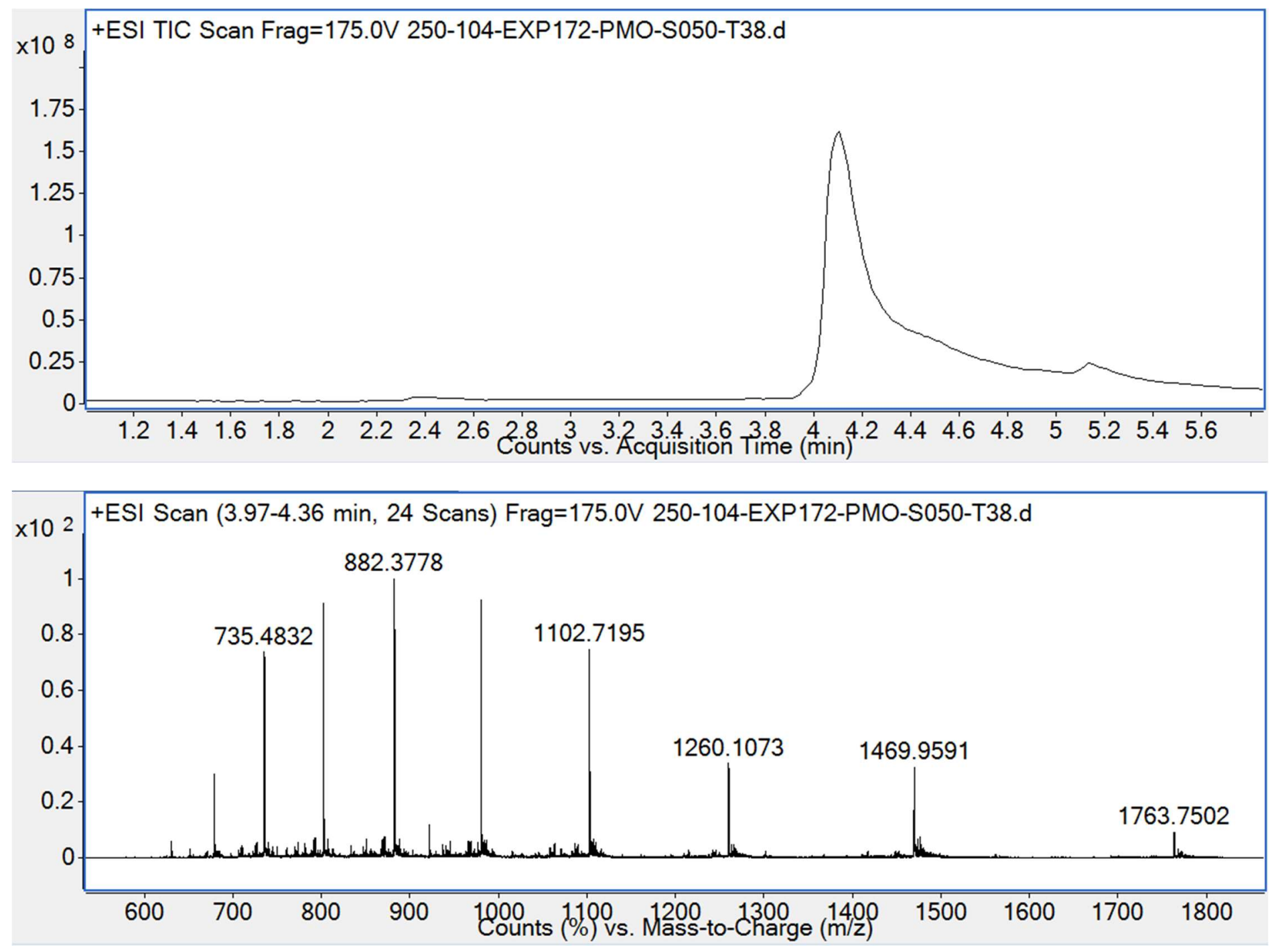


\section{PMO-P17}

Mass expected: $8814.4 \mathrm{Da}$

Mass observed: $8815.1 \mathrm{Da}$

Peptide sequence: KAKAKQBKKKHRWPKXXC
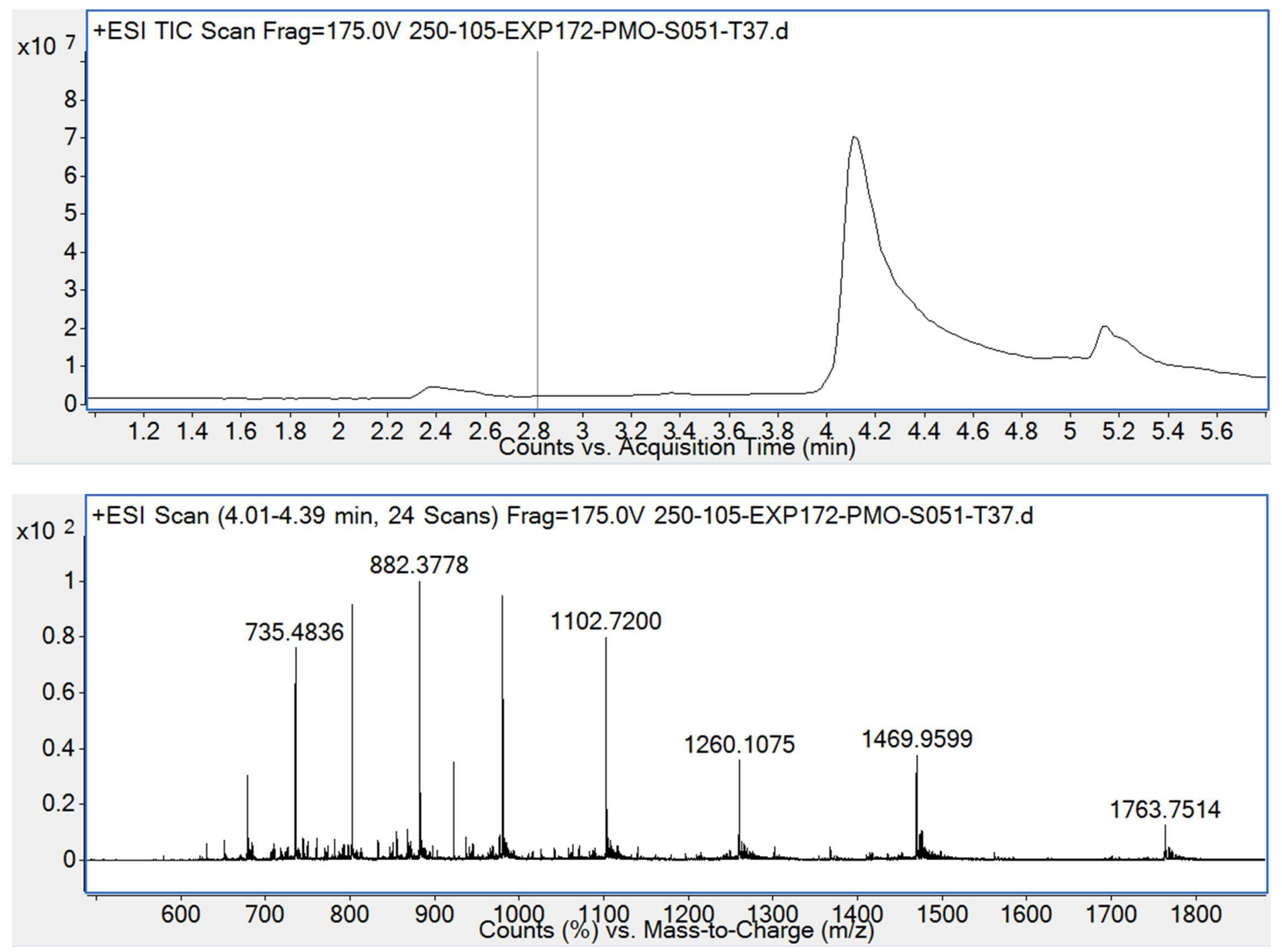


\section{PMO-P18}

Mass expected: $8757.4 \mathrm{Da}$

Mass observed: $8756.7 \mathrm{Da}$

Peptide sequence: KAKAKQBKAKHRWPKXXC
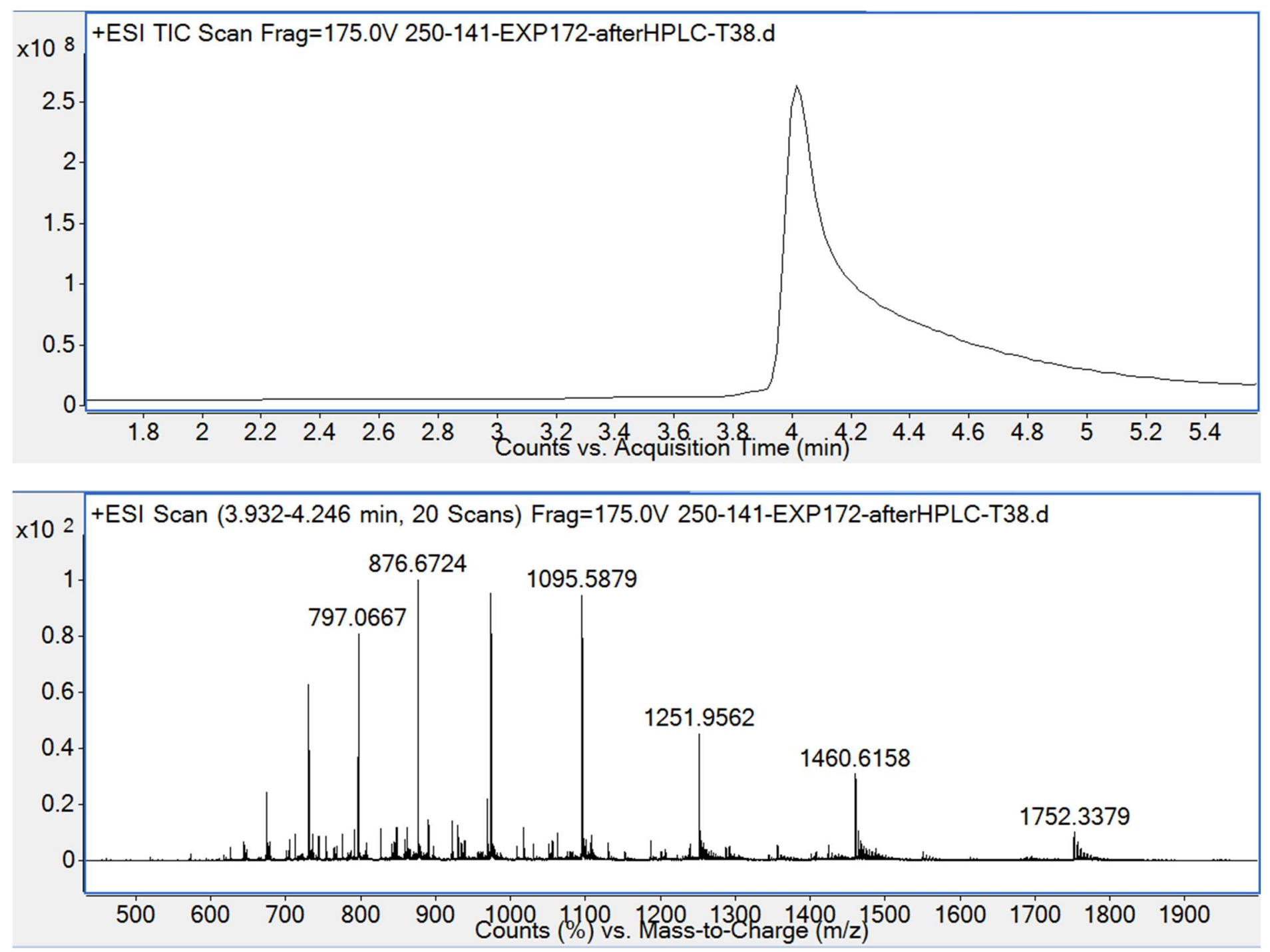


\section{PMO-P19}

Mass expected: $8844.1 \mathrm{Da}$

Mass observed: $8844.3 \mathrm{Da}$

Peptide sequence: KKKKKQBKKKHAWPKXXC
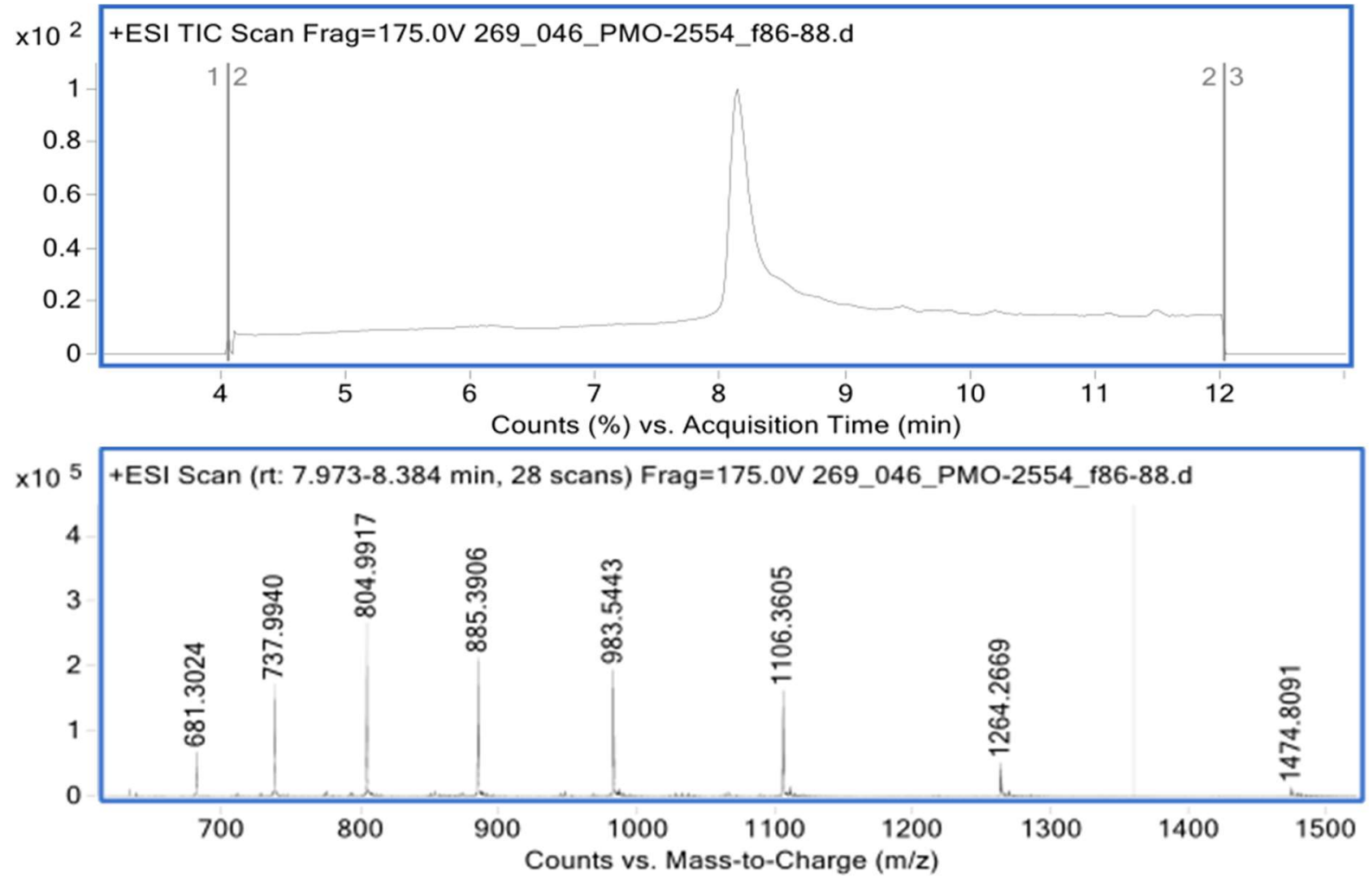


\section{P6-DTA}

Mass expected: $24034.0 \mathrm{Da}$

Mass observed: 24035.0 Da

Peptide sequence: KKKKKQBKKKHRWPKXXC
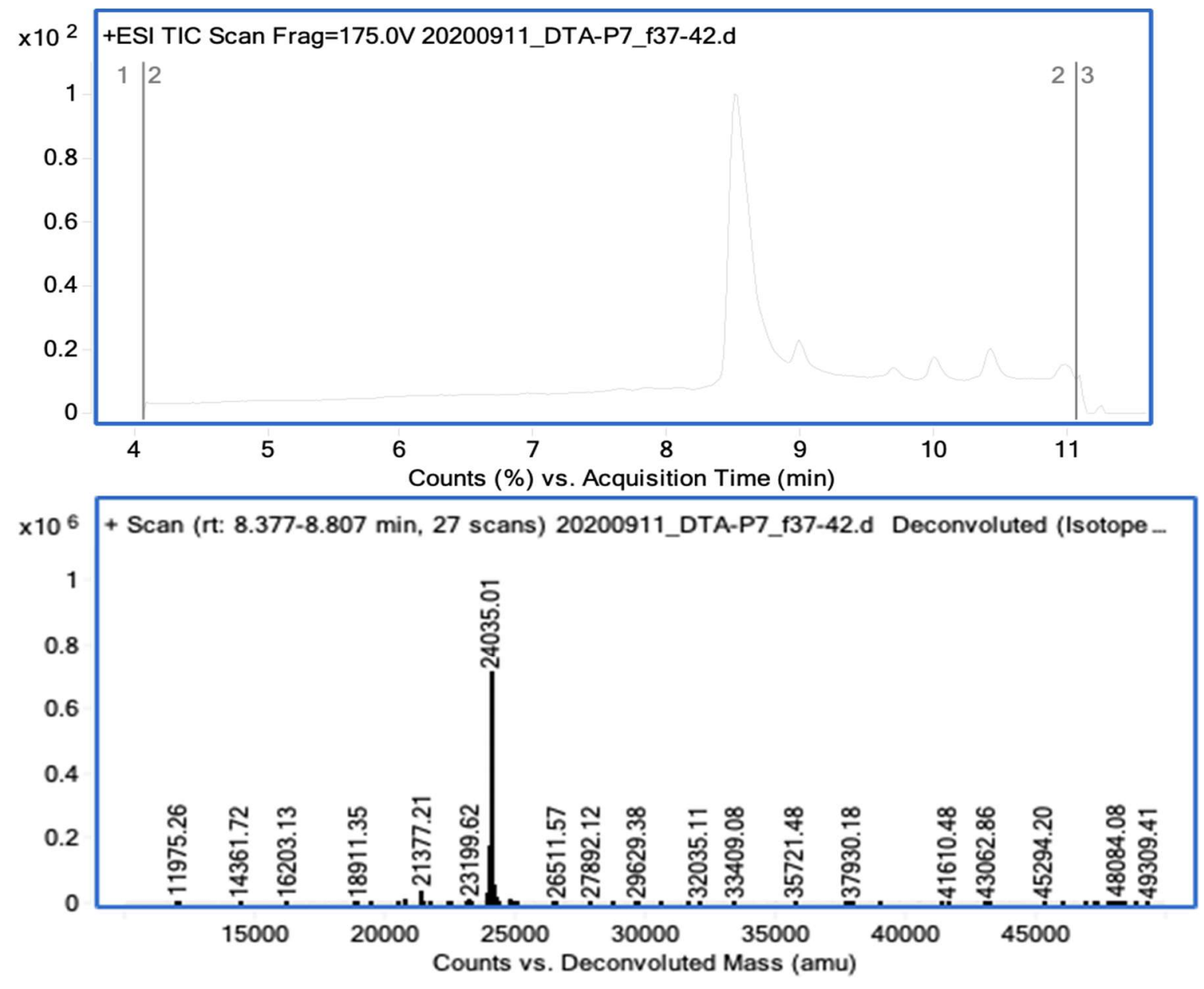


\section{References}

(1) Sazani, P.; Gemignani, F.; Kang, S.-H.; Maier, M. A.; Manoharan, M.; Persmark, M.; Bortner, D.; Kole, R. Systemically Delivered Antisense Oligomers Upregulate Gene Expression in Mouse Tissues. Nat. Biotechnol. 2002, 20 (12), 12281233.

(2) Schissel, C. K.; Mohapatra, S.; Wolfe, J. M.; Fadzen, C. M.; Bellovoda, K.; Wu, C.-L.; Wood, J. A.; Malmberg, A. B.; Loas, A.; Gómez-Bombarelli, R.; Pentelute, B. L. Deep Learning to Design Nuclear-Targeting Abiotic Miniproteins. Nat. Chem. 2021, https://doi.org/10.1038/s41557-021-00766-3.

(3) Wolfe, J. M.; Fadzen, C. M.; Holden, R. L.; Yao, M.; Hanson, G. J.; Pentelute, B. L. Perfluoroaryl Bicyclic CellPenetrating Peptides for Delivery of Antisense Oligonucleotides. Angew. Chem. Int. Ed. 2018, 57 (17), 4756-4759. https://doi.org/10.1002/anie.201801167. 